\title{
Non-invasive assessment of cardiovascular disease : a study of atherosclerotic lesions using biomarkers and MRI
}

Citation for published version (APA):

Lobbes, M. B. I. (2009). Non-invasive assessment of cardiovascular disease : a study of atherosclerotic lesions using biomarkers and MRI. [Doctoral Thesis, Maastricht University]. University Pers Maastricht. https://doi.org/10.26481/dis.20090923ml

Document status and date:

Published: 01/01/2009

DOI:

10.26481/dis.20090923ml

Document Version:

Publisher's PDF, also known as Version of record

Please check the document version of this publication:

- A submitted manuscript is the version of the article upon submission and before peer-review. There can be important differences between the submitted version and the official published version of record.

People interested in the research are advised to contact the author for the final version of the publication, or visit the DOI to the publisher's website.

- The final author version and the galley proof are versions of the publication after peer review.

- The final published version features the final layout of the paper including the volume, issue and page numbers.

Link to publication

\footnotetext{
General rights rights.

- You may freely distribute the URL identifying the publication in the public portal. please follow below link for the End User Agreement:

www.umlib.nl/taverne-license

Take down policy

If you believe that this document breaches copyright please contact us at:

repository@maastrichtuniversity.nl

providing details and we will investigate your claim.
}

Copyright and moral rights for the publications made accessible in the public portal are retained by the authors and/or other copyright owners and it is a condition of accessing publications that users recognise and abide by the legal requirements associated with these

- Users may download and print one copy of any publication from the public portal for the purpose of private study or research.

- You may not further distribute the material or use it for any profit-making activity or commercial gain

If the publication is distributed under the terms of Article $25 \mathrm{fa}$ of the Dutch Copyright Act, indicated by the "Taverne" license above, 


\section{Non-invasive assessment of cardiovascular disease}

A study of atherosclerotic lesions using biomarkers and MRI 
ISBN 9789052788340

(C) Copyright Marc Lobbes, Maastricht 2009

Cover by Datawyse Maastricht

Cover picture: The fortune teller by Simon Vouet, 1617

Press: Datawyse / Universitaire Pers Maastricht 


\section{Non-invasive assessment of cardiovascular disease}

A study of atherosclerotic lesions using biomarkers and MRI

\section{Proefschrift}

ter verkrijging van de graad van doctor aan de Universiteit Maastricht, op gezag van de Rector Magnificus prof. mr. G.P.M.F. Mols

volgens het besluit van het College van Decanen, in het openbaar te verdedigen op woensdag 23 september 2009 om 16.00 uur

door

Marcus Bernardus Irene Lobbes

Geboren 21 november 1977 te Heerlen

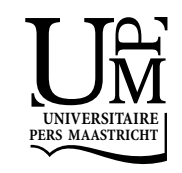


Promotores

Prof. dr. J.M.A. van Engelshoven

Prof. dr. M.J.A.P. Daemen

Copromotores

Dr. M.E. Kooi

Dr. S. Heeneman

Beoordelingscommissie

Prof. dr. A.P.G. Hoeks, voorzitter

Prof. dr. E.A.L. Biessen

Prof. dr. L. Hofstra

Prof. dr. J. Lodder

Prof. dr. W.H. Mess

Financial support by the Netherlands Heart Foundation for the publication of this thesis is gratefully acknowledged. The research described in this thesis was supported by a grant of the Netherlands Heart Foundation (grant number 2002.B033). Financial support by Bayer Schering Pharma for the publication of this thesis is also gratefully acknowledged. 


\section{Content}

Chapter 1 General introduction 9

Chapter 2 Is there more than C-reactive protein and fibrinogen? 23 The prognostic value of soluble CD40 ligand, interleukin 6 , and oxidized low-density lipoprotein with respect to coronary and cerebral vascular disease

Chapter 3 Leukocyte counts, myeloperoxidase and pregnancy associat 41

Chapter 4 Contrast-enhanced magnetic resonanc vessel wall imaging 59 in a rabbit model of therosclerosis: comparison of gadofosveset and Gd-DTPA

Chapter 5 Gadofosveset-enhanced magnetic resonance imaging of human carotid atherosclerotic plaque: initial experience

Chapter 6 General discussion $\quad 97$

Summary $\quad 137$

Nederlandse samenvatting (Dutch summary) 143

Dankwoord 149

Curriculum vitae $\quad 153$

List of publications $\quad 155$ 


$\begin{array}{ll}\text { AMI } & \text { Acute myocardial infarction } \\ \text { APOE-/- } & \text { Apoliprotein-E-deficient } \\ \text { AUC } & \text { Area under curve } \\ \text { B2M } & \text { Beta-2-microglobulin } \\ \text { BNP } & \text { Brain natriuretic peptide } \\ \text { CCL } & \text { Chemotactic cytokine ligand } \\ \text { CE-MRI } & \text { Contrast enhanced magnetic resonance imaging } \\ \text { CEA } & \text { Carotid endarterectomy } \\ \text { CK } & \text { Creatin kinase } \\ \text { CLEAR } & \text { Constant level of appearance } \\ \text { CNR } & \text { Contrast-to-noise ratio } \\ \text { CON } & \text { Controls / controle group } \\ \text { CRP } & \text { C-reactive protein } \\ \text { CSF } & \text { Colony stimulating factor } \\ \text { CT } & \text { Computed tomography } \\ \text { CTA } & \text { Computed tomography angiography } \\ \text { CXCL } & \text { CXC chemokine ligand } \\ \text { DIR } & \text { Double inversion recovery } \\ \text { DSA } & \text { Digital subtraction angiography } \\ \text { EC } & \text { Endothelial cell } \\ \text { ECG } & \text { Electrocardiogram } \\ \text { ECST } & \text { European Carotid Surgery Trial } \\ \text { ELISA } & \text { Enzyme-linked immunosorbent assay } \\ \text { FDA } & \text { Food and Drug Administration } \\ \text { FFE } & \text { Fast field echo } \\ \text { FOV } & \text { Field-of-view } \\ \text { Gd } & \text { Gadolinium } \\ \text { Gd-DTPA } & \text { Gadopentetate dimeglumine } \\ \text { GSM } & \text { Gray scale measurement } \\ \text { HDL-C } & \text { High-density lipoprotein } \\ \text { HE } & \text { Hematoxilin/eosin } \\ \text { HR } & \text { Hazard ratio } \\ \text { HSP } & \text { Heat shock protein } \\ \text { ICAM-1 } & \text { Intracellular adhesion molecule 1 } \\ \text { ICC } & \text { Intraclass correlation coefficient } \\ \text { IFNG } & \text { Interferon-gamma } \\ \text { IL } & \text { Interleukin } \\ \text { LDL-C } & \text { Low-density lipoprotein } \\ & \end{array}$




$\begin{array}{ll}\text { LR } & \text { Likelihood ratio } \\ \text { MMP } & \text { Matrix metalloproteinase } \\ \text { MPO } & \text { Myeloperoxidase } \\ \text { MRA } & \text { Magnetic resonance angiography } \\ \text { MRI } & \text { Magnetic resonance imaging } \\ \text { MSDE } & \text { Motion-sensitized driven equilibrium } \\ \text { NASCET } & \text { North American Symptomatic Carotid Endarterectomy Trial } \\ \text { NCC } & \text { Nested case control (study) } \\ \text { NO } & \text { Nitric oxide } \\ \text { NSA } & \text { Number of signal acquisitions } \\ \text { OR } & \text { Odds ratio } \\ \text { OXLDL } & \text { Oxidized low-density lipoprotein } \\ \text { PAD } & \text { Peripheral artery disease } \\ \text { PAPP-A } & \text { Pregnancy-associated plasma protein A } \\ \text { PAPP-A US } & \text { PAPP-A determined with ultrasensitive research kit } \\ \text { PD } & \text { Proton density } \\ \text { PET } & \text { Positron emission tomography } \\ \text { PIXE } & \text { Proton induced x-ray emission analysis } \\ \text { PROMBP } & \text { Proform of eosinophil major basic protein } \\ \text { REML } & \text { Restricted maximum likelihood } \\ \text { ROI } & \text { Region of interest } \\ \text { ROS } & \text { Reactive oxygen species } \\ \text { RR } & \text { Relative risk } \\ \text { RSI } & \text { Relative signal intensity } \\ \text { SAP } & \text { Stable angina pectoris } \\ \text { SAR } & \text { Specific absorption rate } \\ \text { SCD40L } & \text { Soluble CD40 ligand } \\ \text { SE } & \text { Signal enhancement } \\ \text { SI } & \text { Signal intensity } \\ \text { SMC } & \text { Smooth muscle cell(s) } \\ \text { SNR } & \text { Signal-to-noise ratio } \\ \text { SPECT } & \text { Single photon emission computed tomography } \\ \text { Tc } & \text { Technetium } \\ \text { TE } & \text { Echo time } \\ \text { TFE } & \text { Turbo field echo } \\ \text { TI } & \text { Inversion time } \\ \text { TIA } & \text { Transient ischemic attack } \\ \text { TOF } & \text { Time-of-flight } \\ \text { TR } & \text { Repetition time } \\ \text { TSE } & \text { Turbo spin echo } \\ & \end{array}$


UAP Unstable angina pectoris

US Ultrasound

USPIO Ultrasmall particle of iron oxide

VCAM-1 Vascular cell adhesion molecule 1

VEGF Vascular endothelial growth factor 


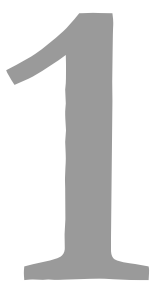

General introduction 


\section{ATHEROSCLEROSIS}

Atherosclerosis is considered to be a chronic inflammatory disease of the large arteries [1, 2]. Atherosclerotic lesions, so called plaques, can progress during decades and may eventually rupture, causing severe clinical complications. These clinical complications, such as myocardial infarction or stroke, are an important cause of mortality, morbidity, and disability.

Atherosclerosis is a main cause of death in industrialized countries. Data from the Dutch Heart Foundation estimated that in 2004 cardiovascular disease was the contributing or underlying cause of 45,000 deaths in the Netherlands (about one third of all Dutch deaths in 2004). In addition, 11,000 deaths occur annually after a stroke in the Netherlands [3].

However, mere attention for mortality alone underestimates the true impact of atherosclerosis on health care. Mortality due to atherosclerosis has decreased in the past decennia due to identification of risk factors and improved medical care. In contrast to the decrease in mortality, the number of patients with cardiovascular disease has increased due to increasing ageing of the population and increased prevalence of people with risk factors for cardiovascular disease, like obesity, hypertension, and diabetes. In short, more people survive atherosclerosis-related complications, but there is an increase in atherosclerosis-associated morbidity and/or disability, placing a huge burden on health care costs [4].

\section{Atherosclerotic lesion development}

The normal arterial vessel wall consists of three main layers. The outermost layer is the tunica adventitia, which consists of (myo)fibroblasts and connective tissue, giving structural support to the artery and providing it with oxygen and nutrients through small 'arteries' within the arterial vessel wall itself (vasa vasorum). The central layer, the tunica media, contains elastic tissue in addition to SMC. The innermost layer is called the tunica intima and consists of connective tissue and more SMC. A small, single layer of cells separates the tunica intima from the circulating blood and is called the endothelial layer. This line of EC's plays a pivotal role in the pathogenesis of atherosclerosis.

Initial atherosclerotic lesion development, which in this early stage can only be detected microscopically, usually starts in the first decade of life. Lesions continue to grow and develop throughout the second, third, and fourth decade of life, until they give rise to clinical complications such as myocar- 
dial infarction, peripheral artery disease, or stroke [5]. These clinical complications of atherosclerosis usually occur after the fifth decade of life.

Dysfunction of the endothelial layer is an important trigger in initiating atherosclerotic lesion development in the vessel wall and can be caused by several pathophysiological factors, but also by hemodynamic factors. Relevant pathophysiological factors are inflammation by bacteria and/or viruses, cytokines or bacterial products, chronic exposure to hyperhomocysteinemia or hypercholesterolemia, stimulation by advanced glycation endproducts, and accumulation of oxidized lipoproteins [6]. Next to these factors, hemodynamic factors are thought to play a prominent role in the initiation of endothelial dysfunction. The most important hemodynamic factor in the pathogenesis is shear stress. Shear stress, defined as the frictional force caused by the flow of viscous blood, is applied to the endothelial layer of the vessel wall [7]. High shear stress $\left(>15\right.$ dyne $\left./ \mathrm{cm}^{2}\right)$ has atheroprotective properties, whereas low shear stress $\left(<4\right.$ dyne $\left./ \mathrm{cm}^{2}\right)$ is usually present at atherosclerosis-prone sites. Areas with low shear stress can be found at inner walls of vessel bifurcations and points of blood flow recirculation and stasis, e.g. coronary arteries, carotid, and aortic bifurcation, which indeed have shown to be highly susceptible for the development of atherosclerosis. Low shear stress reduces the production of endothelial nitric oxide synthase, decreasing the amount of NO in the vessel wall. NO is responsible for several atheroprotective functions in the vessel wall, like EC repair. In addition, low shear stress increases ROS. All these factors contribute to endothelial dysfunction [8].

Dysfunction of the endothelium results in an increased endothelial permeability and expression of adhesion molecules. Circulating LDL can passively enter and leave the vessel wall because of this increased permeability. It can undergo several modifications in the vessel wall, of which oxidation into oxLDL, for example by oxidative cell waste or increased ROS, is the most important one [9]. OxLDL in turn is unable to diffuse out of the vessel wall, resulting in an accumulation of oxidized lipids inside the vessel wall. In addition, adhesion molecules like VCAM-1 attract leucocytes to the affected site and enable monocytes to enter the vessel wall, causing an inflammatory response at the initial atherosclerotic site. In the vessel wall, monocytes transform into macrophages, which in their turn take up the oxLDL, resulting in large, lipid-rich macrophages in the vessel wall, called foam cells. These foam cells produce an abundance of chemokines, pro-inflammatory cytokines, and growth factors. The chemokines and cytokines amplify the in- 
flammatory response in the vessel wall, attracting more leucocytes to the site and increasing the endothelial dysfunction. The growth factors cause SMC to proliferate and migrate, especially in more advanced lesions. In addition, these SMC produce fibrous tissue and collagens. In this way, the lipid deposits are engulfed by a fibrous layer of SMC, giving rise to the typical aspect of the advanced atherosclerotic plaque: a core filled with lipid and necrotic cells, separated from the lumen by a fibrous cap (Figure 1) [10,11].

In atherosclerosis, a process called outward remodelling initially prevents a decrease in lumen diameter. Due to outward remodelling, the plaque can grow without causing significant stenosis of the blood vessel. This process is caused by the shear-responsive production of endothelial NO and MMP's, like MMP-2 and MMP-9 [12]. Angiography (to determine the extent of atherosclerotic disease) can then become contradictory: the lumen diameter can appear normal on angiography, but plaque burden can be extensive at this region of interest. In addition, outward remodelling is associated with an increased risk of plaque rupture, because of the increased production of fibrous cap degrading MMP's in combination with the increased amount of inflammatory cells present in the vessel wall [13]. Therefore, other diagnostic modalities (preferably non-invasively) to determine true plaque burden need to be investigated for their diagnostic potentials.

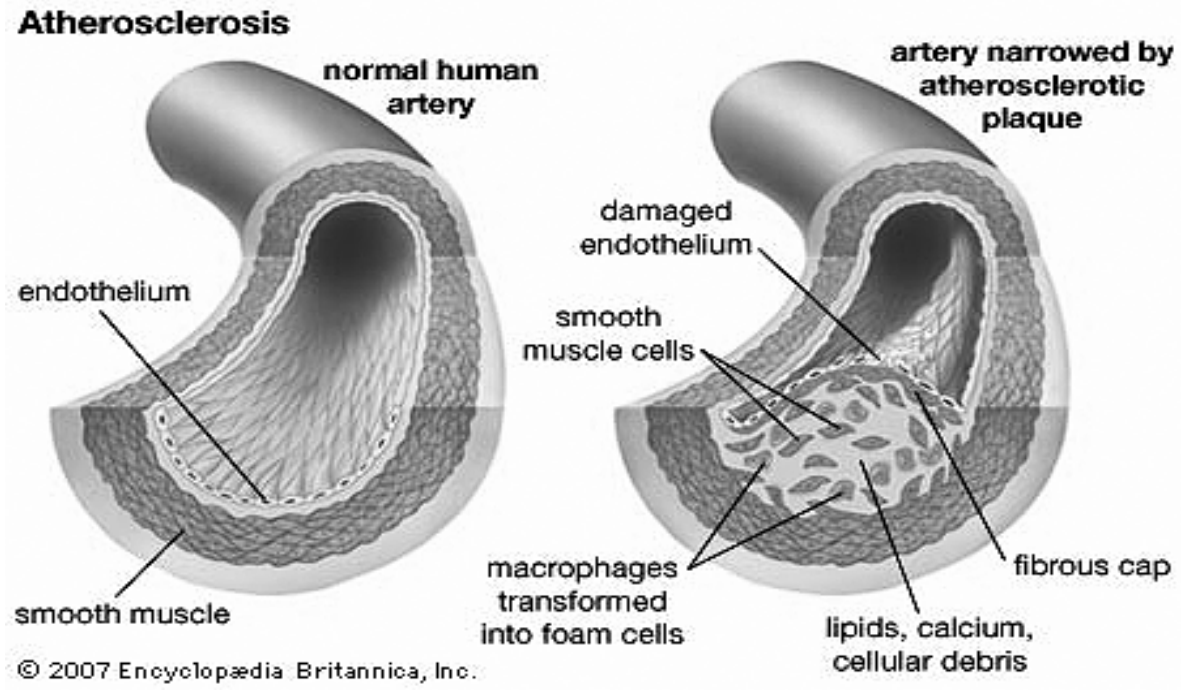

Figure 1. Atherosclerotic plaque development. By courtesy of Encyclopedia Brittanica, Inc., copyright 2007; used with permission. 


\section{Atherosclerotic plaque vulnerability}

Clinical manifestations, e.g. myocardial infarction or stroke, occur when the protective fibrous cap of an atherosclerotic plaque ruptures, exposing the highly thrombogenic contents to the blood stream. This causes thrombus formation which in turn can lead to partial or complete occlusion of the artery.

Partial occlusions, in which the luminal thrombus is again encapsulated by the vessel wall, do not result in the clinical manifestations mentioned above. They are the main cause of so-called silent ruptures. However, the subsequent repair process can result in a further decrease of lumen diameter, which in turn can be easily occluded during a next event of plaque rupture [14]. In PAD or coronary arteries, reduction in lumen diameter can be the cause of exercised-induced ischemia of distal myocardial tissue.

When total occlusion occurs, the distal tissue is deprived of oxygen and nutrients, which leads to functional loss of this tissue. This process can occur in both the heart or the brain, causing myocardial infarction or stroke, respectively [4].

Atherosclerotic plaques with a high susceptibility of causing symptoms are called vulnerable plaques [15]. These vulnerable plaques are characterized by a large necrotic lipid core with an thin fibrous cap, increased inflammation, and intraplaque capillaries $[14,16]$.

In addition, erythrocytes are deposited in the vessel wall due to intraplaque haemorrhaging through disruption of neovascularisation of intraplaque microvessels in advanced atherosclerotic plaques. These intraplaque microvessels are small, immature, leaky blood vessels which supply nutrients to advanced atherosclerotic lesions. They are formed under the influence of hypoxial triggers when plaque size is too large for the vasa vasorum to supply it with nutrients and oxygen [17]. Therefore, neovascularisation density can indirectly be seen as one of the determinants of plaque vulnerability. The cell membranes of these erythrocytes contain a great amount of cholesterol and their degradation within the plaque results in accelerated lipid core progression. At present, knowledge of plaque vulnerability has been largely based on post-mortem studies [14]. 


\section{CLINICAL ASSESSMENT OF PLAQUE VULNERABILITY}

To identify the risk factors that are associated with atherosclerosis and its cardiovascular complications, several risk assessment models have been developed over the past decades. Many of these risk assessment models (such as MONICA and Framingham) are based on traditional risk factors that are associated with the development of atherosclerosis [15]. In these large population studies, it was demonstrated that sex, age, hypercholesterolemia, smoking, hypertension, and diabetes mellitus were all positively related to the development of atherosclerosis and its clinical complications [18]. Although these models can accurately assess long-term outcome in large populations, they fail to assess the individual short-term patient risk.

In papers by Naghavi et al. it was proposed to define patients at risk of cardiovascular events in the near future as vulnerable patients $[15,19]$. These publications were co-authored by a large panel of experts in the field of atherosclerosis and cardiovascular disease. Patient vulnerability is determined by the presence of vulnerable plaques, vulnerable blood (i.e. thrombogenic blood), and/or a vulnerable myocardium (i.e. susceptible for ischemia). Vulnerable patients have a high susceptibility of getting near-future atherosclerosis-related complication and thus might benefit most from aggressive treatment and/or interventions.

To identify this specific population, Fuster proposed a speculative roadmap, which was recently modified by Naghavi et al. [15, 19, 20]. With the aid of questionnaires, participants with an increased risk of cardiovascular events are referred to a physician and with additional screening (e.g. assessment of certain biomarker concentrations) participants are selected with severely increased risk of cardiovascular events. They can be referred to specialized care for further assessment using non-invasive imaging modalities (such as (molecular) MRI) or, eventually, with invasive image modalities that also enable therapeutic interventions. In this paper the need for the development of non-invasive and accurate screening tools was emphasized [19]. In the present thesis, we focused on two aspects of this roadmap, namely noninvasive risk assessment, i.e. validation of (potential) biomarkers, and contrast-enhanced magnetic resonance imaging (CE-MRI) of atherosclerotic plaques. 


\section{Biomarkers for atherosclerotic plaque vulnerability}

Research on the pathogenesis of atherosclerosis has revealed important roles for a great number of soluble molecules throughout the process of plaque growth. Nowadays, some of these molecules can be measured with sensitive assays, enabling the investigation of the association between serum concentrations of these molecules and the occurrence of different forms of cardiovascular disease. The association between these serum biomarkers and cardiovascular disease has been established for a number of molecules, such as CRP and fibrinogen [21].

The association between cardiovascular disease and CRP and fibrinogen has been described extensively $[22,23]$. Their prognostic value for cardiovascular events or mortality has been reviewed in several meta-analyses [23-26]. It was demonstrated that the prognostic value of both CRP and fibrinogen was relatively limited (relative risk estimates ranging 1.4 to 1.8$)$ [23, 27], challenging us to search for other, better predictors of atherosclerosisrelated events.

An increasing number of molecules are eligible, but although the results of some biomarkers are promising on a population level, no single biomarker has yet been able to predict individual cardiovascular risk. With respect to this thesis we performed a systemic review of available literature to assess whether biomarkers, other than the well analyzed and validated CRP and fibrinogen, are able to predict near-future cardiovascular complications. In addition, we compared several new and emerging biomarkers of cardiovascular disease and compared their prognostic value with well validated ('old') biomarkers. Finally, we analyzed which (combination) of the examined biomarkers has the highest potential in predicting future cardiovascular events.

\section{Imaging of atherosclerosis}

Improvements in diagnostic imaging are being developed constantly. At present, the degree of stenosis is determined by DSA in the coronary arteries, or by Duplex ultrasound, CTA, and MRA in the carotid arteries, assuming that a significant stenosis of the artery is associated with increased plaque vulnerability. As mentioned in the previous paragraphs, plaque composition is expected to be a more accurate determinant of plaque vulnerability than determining lumen diameter. Therefore, new imaging modalities to 
visualize plaque composition need to be developed to assess individual plaque vulnerability.

MRI is able to measure lumen diameter as well as plaque components [2830]. Its main advantages are the excellent soft tissue contrast, which is needed to distinguish between plaque components, the absence of ionizing radiation, and its non-invasive nature. Disadvantages of MRI in plaque imaging are the inability to image patients with metal objects in the area that is to be examined (for instance pacemakers in cardiovascular patients) and the potential claustrophobic effect of the MRI bore (tunnel). In addition, imaging of atherosclerotic lesions in small arteries, like coronary arteries, can be challenging due to the limited spatial resolution and relatively low SNR of MRI.

MRI utilizes the magnetic properties of protons to create images of different tissues. These tissues all have characteristic $T_{1}$ and $T_{2}$ relaxation times. By varying MR scan parameters like repetition time (TR) and echo time (TE), images can be generated in which tissue contrasts are primarily determined by differences in $T_{1}$ or $T_{2}$ relaxivity. These MR images are then called $T_{1}$ - or $T_{2}$-weighted images, respectively. Proton density (PD) weighted images can be acquired by generating images with little $\mathrm{T}_{1}$ and $\mathrm{T}_{2}$ contrast [31].

In order to accurately visualize different plaque components within a lesion, multi-sequence, high-resolution MR images are required. Images can be acquired with high (TOF sequences) or low (DIR) or MSDE sequences) signal intensity of the luminal blood [30, 32]. A TOF sequence can be used to distinguish between lumen and (calcified) vessel wall and to evaluate fibrous cap status [33, 34]. DIR sequences utilize inversion pulses to null the blood signal, enabling optimal contrast between lumen and arterial vessel wall [32]. Recently, Wang et al. demonstrated improved nulling of the blood signal by using a MSDE turbo spin echo sequence [35]. Diffusion-weighted imaging analyses the motion of water molecules and can be additionally applied to distinguish between plaque components, but until now, long acquisition times prevented human applications [36].

Finally, CE-MRI can be applied to increase SNR and contrast-to-noise ratio, which might enable imaging of atherosclerotic plaques in even small arteries. The most frequently used contrast agents in plaque imaging are gadolinium (Gd) based agents. In imaging of atherosclerosis, MR signal enhancement after contrast administration has been linked with neovascularisation, 
inflammation, and presence of fibrous tissue [37-40]. New (blood pool) contrast agents, like gadofosveset, have recently become available for clinical applications [41]. However, the potential of gadofosveset in atherosclerotic plaque imaging has yet to be investigated.

With respect to this thesis, we investigated the potential gadofosvesetenhanced MR plaque imaging by comparing post-contrast signal enhancement of the aortic vessel wall in both atherosclerotic and control rabbits. In addition, we compared the results of gadofosveset with the commonly used contrast agent Gd-DTPA. Furthermore, we investigated the potential of gadofosveset-enhanced plaque imaging in an in vivo human carotid artery study, where we compared post-contrast signal enhancements between symptomatic and asymptomatic patients.

\section{MOLECULAR IMAGING}

Molecular imaging techniques directly or indirectly monitor and record the spatio-temporal distribution of molecular or cellular processes for biochemical, biological, diagnostic, or therapeutic applications [42]. Present imaging techniques focus mainly on physiologic or anatomical changes caused by disease. Molecular imaging focuses on specific molecular changes at the preclinical stage of the disease, preventing clinical complications or death. In the general discussion of this thesis, the potential of binding biomarkers and imaging modalities to perform molecular imaging are discussed and future applications are speculated on.

\section{HYPOTHESIS}

In the current thesis, we hypothesize that (novel) biomarkers and (novel) MRI contrast agents can contribute to the improvement of the non-invasive assessment of cardiovascular disease.

\section{OUTLINE OF THIS THESIS}

In chapter 2 , we performed a systematic review to evaluate the prognostic value of serum concentrations of sCD40L, IL- 6 and oxLDL for cardiovascular disease and compared them with the more established biomarkers CRP and fibrinogen. 
In chapter 3, we evaluated the association of new serum biomarkers (such as MPO and PAPP-A) with the presence of different forms of cardiovascular disease (such as stable (SAP) and unstable angina pectoris (UAP) and acute myocardial infarction (AMI)). In addition, we aimed to analyzed which (combination) of the investigated biomarkers has the highest prognostic value for predicting near-future cardiovascular events.

In chapter 4, we investigated the potential of gadofosveset in MR plaque imaging in a rabbit model of atherosclerosis and compared results with the commonly used contrast agent Gd-DTPA.

In chapter 5, we investigated the potential of gadofosveset in MR plaque imaging in patients with atherosclerotic lesions in the carotid arteries.

In chapter 6, the findings as described in this thesis are discussed and future applications are given. 


\section{REFERENCES}

1. Ross R. Atherosclerosis: an inflammatory disease. N Engl J Med. 1999; 340: 115-26.

2. Hansson GK. Inflammation, atherosclerosis, and coronary artery disease. $\mathrm{N}$ Engl J Med. 2005; 352: 1685-95.

3. Jager-Geurts MH, Van Dis SJ, Bots ML. Hart- en vaatziekten in Nederland 2006: cijfers over ziekte en sterfte. 2006, The Hague: Dutch Heart Foundation.

4. Thom T, Haase N, Rosamond W, Howard VJ, Rumsfeld J, Manolio T, Zheng ZJ, Flegal K, O’Donnell C, Kittner S, Lloyd-Jones D, Goff DC Jr, Hong Y, Adams R, Friday G, Furie K, Gorelick P, Kissela B, Marler J, Meigs J, Roger V, Sidney S, Sorlie P, Steinberger J, Wasserthiel-Smoller S, Wilson M, Wolf P, American Heart Association Statistics Committee and Stroke Statistics Committee. Heart disease and stroke statistics: 2006 update: a report from the American Heart Association Statistics Committee and Stroke Statistics Subcommittee. Circulation. 2006; 113: e85-151.

5. Strong JP, Malcolm GT, McMahan CA, Tracy RE, Newman WP 3rd, Herderick EE, Cornill JF. Prevalence and extent of atherosclerosis in adolescents and young adults: implications for prevention from the Pathobiological Determinants of Atherosclerosis in Youth Study. JAMA. 1999; 281: 727-35.

6. Gimbrone Jr MA, Topper JN, Nagel T, Anderson KR, Garcia-Cardeno G. Endothelial dysfunction, hemodynamic forces, and atherogenesis. Ann N Y Acad Sci. 2000; 902: 230-9.

7. Malek AM, Alper SL, Izumo S. Hemodynamic shear stress and its role in atherosclerosis. JAMA. 1999; 282: 2035-42.

8. Cunningham KS, Gotlieb AI. The role of shear stress in the pathogenesis of atherosclerosis. Lab Invest. 2005; 85: 9-23.

9. Lusis AJ. Atherosclerosis. Nature. 2000; 407: 233-41.

10. Libby P, Ridker PM, Maseri A. Inflammation and atherosclerosis. Circulation. 2002; 105: 1135-43.

11. Libby P. Inflammation in atherosclerosis. Nature. 2002; 420: 868-74.

12. Ward MR, Pasterkamp G, Yeung AC, Borst C. Arterial remodeling. Mechanisms and clinical implications. Circulation. 2000; 102: 1186-91.

13. Pasterkamp G, Galis ZS, De Kleijn DP. Expansive arterial remodeling: location, location, location. Arterioscler Thromb Vasc Biol. 2004; 24: 650-7.

14. Virmani R, Kolodgie FD, Burke AP, Farb A, Schwartz SM. Lessons from sudden coronary death: a comprehensive morphological classification scheme for atherosclerotic lesions. Arterioscler Thromb Vasc Biol. 2000; 20: 1262-75.

15. Naghavi M, Libby P, Falk E, Casscells SW, Litovsky S, Rumberger J, Badimon JJ, Stefanadis C, Moreno P, Pasterkamp G, Fayad Z, Stone PH, Waxman S, Raggi P, Madjid M, Zarrabi A, Burke A, Yuan C, Fitzgerald PJ, Siscovick DS, de Korte CL, Aikawa M, Juhani Airaksinen KE, Assmann G, Becker CR, Chesebro JH, Farb A, Galis ZS, Jackson C, Jang IK, Koenig W, Lodder RA, March K, Demirovic J, Navab M, Priori SG, Rekhter MD, Bahr R, Grundy SM, Mehran R, Colombo A, Boerwinkle E, Ballantyne C, Insull W Jr, Schwartz RS, Vogel R, Serruys PW, Hansson GK, Faxon DP, Kaul S, Drexler H, Greenland P, Muller JE, Virmani R, Ridker PM, Zipes DP, Shah PK, Willerson JT. From vulnerable plaque to vulnerable patient: a call for new definitions and risk assessment strategies: Part I. Circulation. 2003; 108: 1664-72.

16. Lutgens E, Van Suylen RJ, Faber BC, Gijbels MJ, Eurlings PM, Bijnens AP, Cleutjens KB, Heeneman S, Daemen MJ. Atherosclerotic plaque rupture. Local or systemic process? Arterioscler Thromb Vasc Biol. 2003; 23: 2123-30. 
17. Virmani R, Kolodgie FD, Burke AP, Finn AV, Gold HK, Tulenko TN, Wrenn SP, Narula $\mathrm{J}$. Atherosclerotic plaque progression and vulnerability to rupture: angiogenesis as a source of intraplaque hemorrhage. Arterioscler Thromb Vasc Biol. 2005; 25: 2054-61.

18. Manhem K, Dotevall A, Wilhelmsen L, Rosengren A. Social gradients in cardiovascular risk factors and symptoms of Swedish men and women: the Goteborg MONICA Study 1995. J Cardiovasc Risk. 2000; 7: 359-68.

19. Naghavi M, Libby P, Falk E, Casscells SW, Litovsky S, Rumberger J, Badimon JJ, Stefanadis C, Moreno P, Pasterkamp G, Fayad Z, Stone PH, Waxman S, Raggi P, Madjid M, Zarrabi A, Burke A, Yuan C, Fitzgerald PJ, Siscovick DS, de Korte CL, Aikawa M, Airaksinen KE, Assmann G, Becker CR, Chesebro JH, Farb A, Galis ZS, Jackson C, Jang IK, Koenig W, Lodder RA, March K, Demirovic J, Navab M, Priori SG, Rekhter MD, Bahr R, Grundy SM, Mehran R, Colombo A, Boerwinkle E, Ballantyne C, Insull W Jr, Schwartz RS, Vogel R, Serruys PW, Hansson GK, Faxon DP, Kaul S, Drexler H, Greenland P, Muller JE, Virmani R, Ridker PM, Zipes DP, Shah PK, Willerson JT From vulnerable plaque to vulnerable patient: a call for new definitions and risk assessment strategies: Part II. Circulation. 2003; 108: 1772-8.

20. Fuster V. Epidemic of cardiovascular disease and stroke: the three main challenges. Presented at the 71st scientific sessions of the American Heart Association. Dallas, Texas. Circulation. 1999; 99: 1132-7.

21. Koenig W, Khuseyinova N. Biomarkers of atherosclerotic plaque instability and rupture. Arterioscler Thromb Vasc Biol. 2007; 27: 15-26.

22. Ridker PM. High-sensitivity C-reactive protein: potential adjunct for global risk assessment in the primary prevention of cardiovascular disease. Circulation. 2001; 103: 1813-8.

23. Danesh J, Collins R, Appleby P, Peto R. Association of fibrinogen, C-reactive protein, albumin, or leukocyte count with coronary heart disease: meta-analyses of prospective studies. JAMA. 1998; 279: 1477-82.

24. Danesh J, Wheeler JG, Hirschfield GM, Eda S, Eiriksdottir G, Rumley A, Lowe GD, Pepys MB, Gudnason V. C-reactive protein and other circulating markers of inflammation in the prediction of coronary heart disease. N Engl J Med. 2004; 350: 1387-97.

25. Yarnell JW, Patterson CC, Sweetnam PM, Lowe GD. Haemostatic/inflammatory markers predict 10 -year risk of IHD at least as well as lipids: the Caerphilly collaborative studies. Eur Heart J. 2004; 25: 1049-56.

26. Smith GD et al. Does elevated plasma fibrinogen increase the risk of coronary heart disease? Evidence from a meta-analysis of genetic association studies. Arterioscler Thromb Vasc Biol. 2005; 25: 2228-33.

27. Fibrinogen Studies Collaboration, Danesh J, Lewington S, Thompson SG, Lowe GD, Collins R, Kostis JB, Wilson AC, Folsom AR, Wu K, Benderly M, Goldbourt U, Willeit J, Kiechl S, Yarnell JW, Sweetnam PM, Elwood PC, Cushman M, Psaty BM, Tracy RP, Tybjaerg-Hansen A, Haverkate F, de Maat MP, Fowkes FG, Lee AJ, Smith FB, Salomaa V, Harald K, Rasi R, Vahtera E, Jousilahti P, Pekkanen J, D'Agostino R, Kannel WB, Wilson PW, Tofler G, Arocha-Piñango CL, Rodriguez-Larralde A, Nagy E, Mijares M, Espinosa R, Rodriquez-Roa E, Ryder E, Diez-Ewald MP, Campos G, Fernandez V, Torres E, Marchioli R, Valagussa F, Rosengren A, Wilhelmsen L, Lappas G, Eriksson H, Cremer P, Nagel D, Curb JD, Rodriguez B, Yano K, Salonen JT, Nyyssönen K, Tuomainen TP, Hedblad B, Lind P, Loewel H, Koenig W, Meade TW, Cooper JA, De Stavola B, Knottenbelt C, Miller GJ, Cooper JA, Bauer KA, Rosenberg RD, Sato S, Kitamura A, Naito Y, Palosuo T, Ducimetiere P, Amouyel P, Arveiler D, Evans AE, Ferrieres J, Juhan-Vague I, Bingham A, Schulte H, Assmann G, Cantin B, 
Lamarche B, Després JP, Dagenais GR, Tunstall-Pedoe H, Woodward M, Ben-Shlomo Y, Davey Smith G, Palmieri V, Yeh JL, Rudnicka A, Ridker P, Rodeghiero F, Tosetto A, Shepherd J, Ford I, Robertson M, Brunner E, Shipley M, Feskens EJ, Kromhout D, Dickinson A, Ireland B, Juzwishin K, Kaptoge S, Lewington S, Memon A, Sarwar N, Walker M, Wheeler J, White I, Wood A. Plasma fibrinogen level and the risk of major cardiovascular diseases and nonvascular mortality: an individual participant metaanalysis. JAMA. 2005; 294: 1799-809.

28. Fayad ZA, Fuster V. Characterization of atherosclerotic plaques by magnetic resonance imaging. Ann N Y Acad Sci. 2000; 902: 173-86.

29. Yuan C, Kerwin WS. MRI of atherosclerosis. J Magn Reson Imaging. 2004; 19: 710-9.

30. Leiner T, Gerretsen S, Botnar R, Lutgens E, Cappendijk V, Kooi E, Van Engelshoven J. Magnetic resonance imaging of atherosclerosis. Eur Radiol. 2005; 15: 1087-99.

31. Weishaupt D, Marincek B. How does MRI work? An introduction to the physics and function of magnetic resonance imaging. 2003, Berlin Heidelberg New York: SpringerVerlag.

32. Yuan C, Kerwin WS, Yarnykh VL, Cai J, Saam T, Chu B, Takaya N, Ferguson MS, Underhill $\mathrm{H}, \mathrm{Xu} \mathrm{D}$, Liu F, Hatsukami TS. MRI of atherosclerosis in clinical trials. NMR Biomed. 2006; 19: 636-54.

33. Yuan C Zhang SX, Polissar NL, Echelard D, Ortiz G, Davis JW, Ellington E, Ferguson MS, Hatsukami TS. Identification of fibrous cap rupture with magnetic resonance imaging is highly associated with recent transient ischemic attack or stroke. Circulation. 2002; 105: 181-5.

34. Hatsukami TS, Ross R, Polissar N, Yuan C. Visualization of fibrous cap thickness and rupture in human atherosclerotic carotid plaque in vivo with high-resolution magnetic resonance imaging. Circulation. 2000; 102: 959-64.

35. Wang J, Yarnykh VL, Hatsukami T, Chu B, Balu N, Yuan C. Improved suppression of plaque-mimicking artifacts in black-blood carotid atherosclerosis imaging using a multislice motion-sensitized driven-equilibrium (MSDE) turbo spin-echo (TSE) sequence. Magn Reson Med. 2007; 58: 973-81.

36. Qiao Y, Ronen I, Viereck J, Ruberg FL, Hamilton JA. Identification of atherosclerotic lipid deposits by diffusion-weighted imaging. Arterioscler Thromb Vasc Biol. 2007; 27: 1440-6.

37. Kerwin WS, O’Brien KD, Ferguson MS, Polissar N, Hatsukami TS, Yuan C. Inflammation in carotid atherosclerotic plaque: a dynamic contrast-enhanced MR imaging study. Radiology. 2006; 241: 459-68.

38. Wasserman BA, Casal SG, Astor BC, Aletras AH, Arai AE. Wash-in kinetics for gadolinium-enhanced magnetic resonance imaging of carotid atheroma. J Magn Reson Imaging. 2005; 21: 91-5.

39. Aoki S, Aoki K, Ohsawa S, Nakajima H, Kumagai H, Araki T. Dynamic MR imaging of the carotid wall. J Magn Reson Imaging. 1999; 9: 420-7.

40. Weiss CR, Arai AE, Bui MN, Agyeman KO, Waclawiw MA, Balaban RS, Cannon RO 3rd. Arterial wall MRI characteristics are associated with elevated serum markers of inflammation in humans. J Magn Reson Imaging. 2001; 14: 698-704.

41. Goyen M, Shamsi K, Schoenberg SO. Vasovist-enhanced MR angiography. Eur Radiol. 2006; 16 Suppl 2: B9-14.

42. Thakur ML, Lentle BC. Joint SNM/RSNA Molecular Imaging Summit Statement. J Nucl Med. 2005; 46: 11N-13N, 42N. 



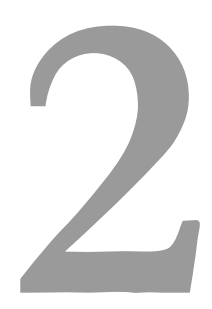

\section{Is there more than CRP and fibrinogen?}

The prognostic value of soluble CD40 ligand, interleukin-6, and oxidized low-density lipoprotein with respect to coronary and cerebral vascular disease

M.B.I. Lobbes, E. Lutgens, S. Heeneman, K.B.J.M. Cleutjens, M.E. Kooi, J.M.A. van Engelshoven, M.J.A.P. Daemen, P.J. Nelemans

Published in: Atherosclerosis 2006; 187: 18-25 


\section{ABSTRACT}

Incidence of atherosclerosis and atherosclerosis-related complications will increase significantly in the coming decennia. Research identified many serum and plasma markers that are associated with cardiovascular disease. However, little is known about the prognostic value of these markers to identify patients at risk for future cardiovascular events. Therefore, we aimed to investigate the prognostic value of three of these markers (sCD40L, IL-6, and oxLDL) with respect to coronary vascular disease and stroke. For this reason the Medline database was searched for the period January 1999 to January 2005. To be selected in our study, concentration of the marker had to be determined at baseline, follow-up period had to be longer than 3 months and an estimate of relative risk had to be available. Based on these criteria, 4 studies for sCD40L, 10 for IL- 6 and 2 for oxLDL were selected. Relative risk estimates adjusted for potential confounders varied between 1.9 and 2.8 for sCD40L, between 1.1 and 3.1 for IL-6, and between 1.9 and 3.2 for oxLDL. In conclusion, this systematic review shows that $\mathrm{sCD} 40 \mathrm{~L}, \mathrm{IL}-6$ and oxLDL are associated with an increased relative risk of developing cardiovascular disease. 


\section{INTRODUCTION}

Atherosclerosis is considered to be a chronic inflammatory disease of the large arteries [1]. Until today, it remains the main cause of deaths in industrialized countries. Atherosclerotic plaques can progress and eventually rupture, causing severe clinical complications, such as myocardial infarction or stroke. Besides mortality these complications are the cause of significant morbidity and disability, resulting in a huge burden on costs on health care.

Therefore, the need for early identification and treatment of atherosclerosis is becoming more and more important. A transition from a curative to a preventive treatment strategy in cardiovascular disease is essential to restrict costs that are associated with atherosclerosis-related complications.

Consequently, recent research has focused on the identification of new risk factors for cardiovascular disease, especially on the prognostic value of serum markers of inflammation. For example, Ridker et al. showed that in apparently healthy women, CRP concentrations are a strong independent risk factor for cardiovascular disease [2]. In another study, Yarnell et al. demonstrated that some inflammatory markers, like fibrinogen, are predictors of subsequent ischemic heart disease in a population of 4860 men [3]. Although the prognostic value of CRP and fibrinogen has been confirmed in recent large meta-analyses [4-6], little is known about the prognostic value of other markers, such as sCD40L, IL-6, and oxLDL.

These markers have all proved to be important players in atherosclerosis. Thus, several components of the CD40-CD40L dyad are present in the majority of plaque cell-types, and expression is most pronounced in advanced and ruptured (human) atherosclerotic plaques. Furthermore, it was demonstrated that both early and delayed treatment with anti-CD40L antibody resulted in the development of a more stable plaque phenotype in ApoE-/- mice [7-9]. In addition, concentrations of both membrane-bound and soluble forms of CD40L were increased in patients with angina pectoris [10]. The association between IL-6 and cardiovascular diseases was demonstrated in several studies [11, 12]. Maier et al demonstrated increased levels of IL-6 at the site of plaque rupture, indicating a significant role for IL-6 in atherosclerosis and plaque destabilization [13]. Schieffer et al. demonstrated that a life-time deficiency of IL- 6 in mice enhanced atherosclerotic plaque formation, but decreases the accumulation of inflammatory cells in atherosclerotic plaques [14]. Finally, it has been demonstrated that severe acute 
coronary lesions also contain more macrophages rich in oxLDL [15]. In addition, multiple studies have demonstrated the association between elevated concentrations of oxLDL and cardiovascular diseases [15-17]. In conclusion, it was demonstrated that sCD40L, IL-6 and oxLDL have a positive association with cardiovascular disease. However, little is known about their prognostic value with respect to cardiovascular diseases.

Therefore, the aim of this systematic review is to provide an overview of available literature on the prognostic value of the markers sCD40L, IL-6, and oxLDL in cardiovascular disease. We suggest that sCD40L, IL-6, and oxLDL are also potential risk markers, but to the best of our knowledge, their prognostic value has never been summarized in a meta-analysis or systematic review.

\section{METHODS}

\section{Literature search}

The Medline database was used to search for any available literature on the prognostic value of these markers for cardiovascular disease, published in the period of January 1999 to January 2005. Search terms were: soluble CD40 ligand, sCD40L, interleukin-6, IL-6, oxidized LDL, oxLDL, cardiovascular disease, coronary heart disease, myocardial infarction, stroke, angina, and atherosclerosis. To identify additional relevant references, the references of the publications retrieved were checked manually.

\section{Study selection}

Studies were selected from the Medline results by reading the title of the study. From these studies one reviewer (M.L.) selected potential eligible studies by reading the abstracts. Studies were selected for review if they fulfilled all of the following inclusion criteria: concentration of the marker had to be measured before occurrence of the cardiovascular outcome of interest, follow-up period had to be at least three months and an estimate of relative risk had to be presented. The term relative risk (RR) estimate encompasses relative risk, odds ratio or hazard ratio.

The cardiovascular diseases of interest were stable angina pectoris, unstable angina pectoris, myocardial infarction and stroke. Studies focussing solely on congestive heart failure were excluded from review. However, when 
congestive heart failure was one of the cardiovascular diseases of interest next to the ones mentioned above, the study was included. Studies which used the same study population were used only once. In this case, the study with the most details or the highest number of participants was included in the review.

\section{Data extraction}

Two reviewers (M.L., E.L.) independently extracted relevant data such as study results and characteristics from each selected publication using a standardized extraction form. The reviewers were not blinded to names of the authors, name of the journal and year of publication. In cases of discrepancies between the two reviewers, a third reviewer (P.N.) read the paper and consensus opinion was used for analysis.

The following study characteristics were recorded: (a) type of marker under study, (b) method of marker measurement, (c) type of study population: free or not free from cardiovascular disease, (d) cardiovascular outcome(s) under study, (e) study design, and (f) minimal duration of the follow-up period. With respect to the analysis, the following data were recorded: (a) number of participants, (b) exposure categories and reference category, (c) confounders for which the analysis was adjusted, and (d) type of analysis (Table 1). If results were presented for multiple cardiovascular outcomes, we extracted the relative risk estimates associated with the cardiovascular outcomes of primary interest, being atherosclerosis-related complications, such as myocardial infarction, stroke and (cardiovascular) mortality.

\section{Statistical analysis}

A distinction was made between 1) crude RR estimates, 2) RR estimates adjusted for potential confounders, excluding other markers and 3) RR estimates adjusted for potential confounders, including other markers (Table 2). The latter RR estimates best reflect the independent contribution of the specific marker to the probability of developing cardiovascular disease.

When reviewing observational studies, it is expected that heterogeneity of populations, designs, outcome measurements and analysis will result in variation between study results that cannot be accounted for by sampling variation. For this reason, we refrained from pooling, because we considered a single summary measure to be inappropriate. 
Forest plots of RR estimates of included studies and corresponding 95\% confidence intervals (if available) are used to give a graphical display of the results from individual studies.

\section{RESULTS}

\section{General results}

The total number of identified publications differed for each marker under study: 63 for sCD40L, 2691 for IL-6, and 1226 for oxLDL. After reading the abstracts, the number of publications that was potentially eligible was 6 for sCD40L, 15 for IL-6, and 11 for oxLDL. A total of 20 publications remained after applying the in- and exclusion criteria: 6 for sCD40L [18-23], 11 for IL-6 [24-34], and 3 for oxLDL [35-37].

Four of these publications were additionally excluded [19, 21, 26, 35], because they partly duplicated the study population used in other publications, leaving 16 publications for review.

The large number of excluded studies was mainly due to the fact that we encountered many case-controlled, cross-sectional studies which compared mean concentrations of the selected markers in subjects with and without cardiovascular disease. Although these studies demonstrate that mean concentrations were generally significantly higher in patients with cardiovascular disease (cases) than in controls, they were excluded from review since they failed to provide an estimate of the magnitude of relative risk associated with elevated concentrations of serum marker.

\section{Results}

Table 1 presents the study characteristics from studies included in our review. Table 2 presents the crude and adjusted relative risk estimates of these studies. Four studies on sCD40L, ten studies on IL-6, and two studies on oxLDL were included for review. Eight of the sixteen selected studies were nested case-control studies within a cohort of patients for whom baseline measurements of markers were available. Subjects who developed the cardiovascular outcome of interest during follow-up were considered to be cases. They were compared to subjects not developing the cardiovascular outcome of interest. The remaining eight studies were all prospective cohort studies, in which participants were categorized into exposure categories 
according to the baseline concentration of the markers. The participants in these different categories were monitored and compared for the occurrence of the cardiovascular outcome of interest.

The studies showed large differences in study population, duration of follow-up, cardiovascular outcome of interest and exposure categories which were compared. Duration of the follow-up varied between 6 months and 8 years. Most studies evaluated the prognostic value of markers in participants who at baseline were free of cardiovascular disease, but in a few studies the study population consisted (totally or partly) of participants who already had cardiovascular disease at the time of inclusion.

Primary cardiovascular outcomes under study were atherosclerosis-related complications, such as myocardial infarction, stroke and (cardiovascular) mortality. The majority of the studies used composite events as study outcome (Table 1). One of the publications additionally investigated congestive heart failure as study outcome [37]. Furthermore, combinations of cardiovascular events (e.g. death/myocardial infarction or death/myocardial infarction/congestive heart failure) were also observed [20].

Exposure categorization also differed between the studies: most studies compared subjects with concentrations in the highest quintile (or quartile, or tertile) versus those with concentrations in the lowest quintile (or quartile, or tertile). A few studies compared subjects with a concentration exceeding a specific cut-off point versus those with a concentration below that cut-off point. In addition, these cut-off points varied between studies. There were also differences in the degree of adjustment for potential confounders. Almost all of the studies adjusted for the most relevant cardiovascular risk factors, e.g. age, sex, and smoking. Some studies restricted the study population of interest to either men or women [18, 25, 29-31]. One study investigated two cohorts of which one consisted solely of men and one solely of women [34]. Most studies additionally adjusted for other risk factors of cardiovascular disease, but there was a large variation in the number and type of cardiovascular risk factors which were taken into account. In most cases, additional adjustment was performed for confounders like hypertension, body mass index, low (or high) density lipoprotein levels and history of cardiovascular disease. 


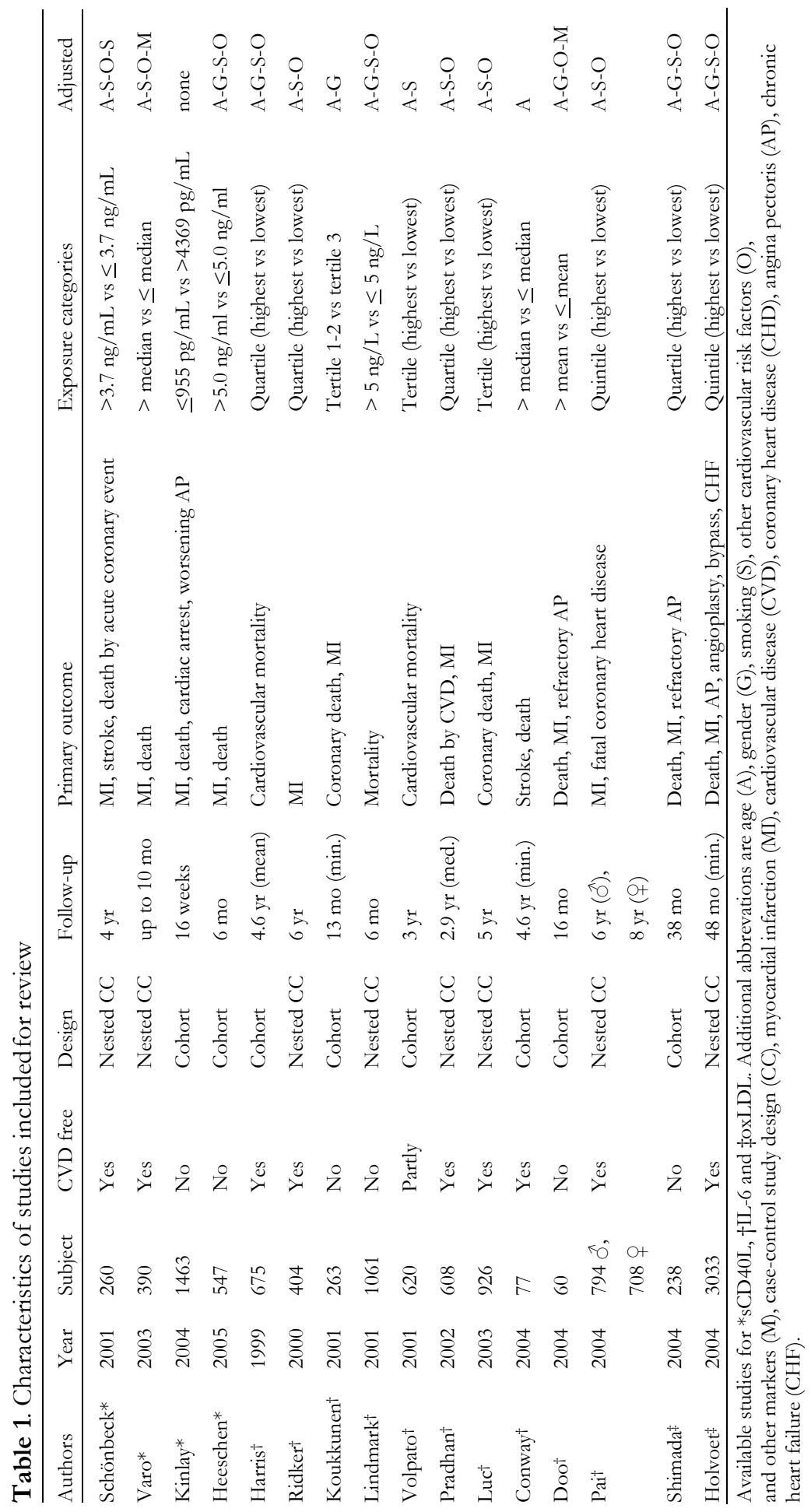


Table 2. Crude and adjusted relative risk estimate and 95\% confidence intervals for the primary study outcome. *For MI, also adjusted for CRP and troponin. † Also adjusted for troponin, CRP, PAPP-A, VEGF, and IL-10. $\$$ Adjusted for troponin and CRP. $\S$ Not significant, also adjusted for anti-oxLDL antibody, VCAM-1, ICAM-1, E-selectin, and CRP. || Not significant. Additional abbreviations are: relative risk (RR), hazard ratio (HR), and odds ratio (OR).

\begin{tabular}{llll}
\hline \multirow{2}{*}{ Author } & \multicolumn{2}{l}{ Relative risk estimates (95\% confidence interval) } \\
\cline { 2 - 3 } Crude & Adjusted & RR estimate \\
Varönbeck & $3.3(1.2-8.6)$ & $2.8(0.9-8.0)$ & RR \\
Kinlay & NA & $1.9(\mathrm{NA})^{*}$ & HR \\
Heeschen & $1.86(1.25-2.77)$ & NA & OR \\
Harris & NA & $2.37(1.34-4.18) \dagger$ & OR \\
Ridker & NA & $2.2(1.0-4.8)$ & RR \\
Koukkunen & $2.3(1.3-4.3)$ & $2.3(1.1-4.6)$ & RR \\
Lindmark & $1.71(1.18-2.48)$ & $1.54(1.04-2.28)$ & HR \\
Volpato & $3.19(1.77-5.74)$ & NA & RR \\
Pradhan & $2.99(1.57-6.53)$ & NA & RR \\
Luc & NA & $2.1(1.1-4.0)$ & OR \\
Conway & NA & $3.10(1.77-5.44) \ddagger$ & RR \\
Doo & $2.99(1.34-6.65)$ & $2.91(1.20-6.51)$ & RR \\
Pai (study men) & NA & $1.1(0.7-2.1) \S$ & OR \\
Pai (study women) & NA & $1.31(0.77-2.22) \|$ & RR \\
Shimada & NA & $1.05(0.56-1.97) \|$ & RR \\
Holvoet & NA & $3.15(1.47-6.76)$ & HR \\
\hline
\end{tabular}

The relative risk estimates with 95\% confidence intervals from the included studies are summarized in a Forest plot (Figure 1). All studies show significantly increased relative risk estimates for cardiovascular events at elevated concentrations of the marker under study, except for the studies of Doo and Pai $[30,34]$. These latter studies could not demonstrate a significant increase of the relative risk estimate for cardiovascular disease at increased levels of IL-6.

For sCD40L, a total number of 2660 participants were analysed in two nested case control studies $(n=650)$ and two cohort studies $(n=2010)$. The study population was free from cardiovascular disease in both nested case control studies. Participants in the cohort studies were not free from cardiovascular disease. Follow-up period varied from 16 weeks to 4 years and 
Chapter 2

Biomarker Autho

$\mathrm{N}$

Schönbeck et al. 260

Varo et al.* $\quad 390$

sCD40L

Kinlay et al. $\quad 1463$

Heeschen et al. $\quad 547$

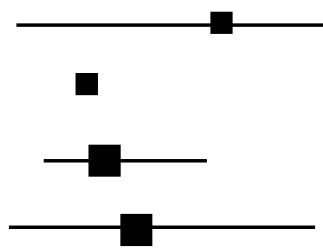

Harris et al. $\quad 675$

Ridker et al. $\quad 404$

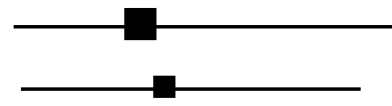

Koukkunen et al. 263

Lindmark et al. $\uparrow 1061$

Volpato et al. $\uparrow \quad 620$

IL-6 Pradhan et al. 608

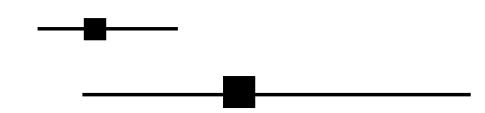

Luc et al.

926

Conway et al.

77

Doo et al.t

60

Pai et al. $(\overline{0}) \ddagger$

794

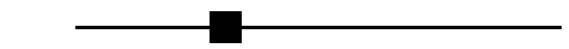

Pai et al. $(+) \ddagger$

708

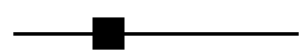

26

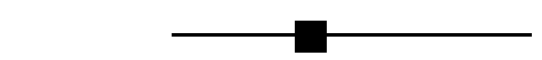

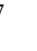
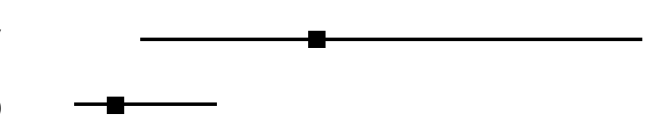
For IL-6, a total number of 6196 participants were analysed in five nested case control studies $(\mathrm{n}=4501)$ and five cohort studies ( $\mathrm{n}=1695)$. In six studies, the study population was free from cardiovascular disease. One study analysed a study population that was partly free of cardiovascular disease. The remaining three studies analysed study populations not free of cardiovascular disease. The follow-up period varied between 6 months and 8 years and the most used study outcome was (cardiovascular) mortality. Myocardial infarction, revascularization, admission for an acute CHD event, stroke and refractory angina pectoris were additional study outcomes. The adjusted relative risk estimates for IL-6 varied between 1.1 and 3.1.

Finally, a total number of 3271 participants were analysed for oxLDL. One nested case control study $(\mathrm{n}=3033)$ and one cohort study $(\mathrm{n}=238)$ was reviewed. The nested case control study consisted of a study population free from cardiovascular disease. In contrast, the cohort study analysed a study population that already suffered from cardiovascular disease. Follow-up period varied from a mean of 38 months to a minimum of 48 months, with death, myocardial infarction and (refractory) angina pectoris as the most prominent study outcomes. Holvoet et al. [37] additionally analysed angioplasty, bypass surgery and chronic heart failure as study outcome of interest. The adjusted relative risk estimates varied between 1.9 and 3.2.

\section{DISCUSSION}

The aim of this systematic review was to investigate whether there were additional serologically determinable markers besides CRP and fibrinogen that have a prognostic value for future cardiovascular events. Results show that with the exception of two studies, all studies showed a significantly increased relative risk for developing future cardiovascular events in patients with elevated levels of sCD40L, IL-6 or oxLDL at baseline. These results pertain to both subjects free of cardiovascular disease and subjects already suffering from cardiovascular disease. RR estimates remained increased after adjustment for cardiovascular risk factors. To the best of our knowledge, this is the first systematic review of the available literature on this subject for the markers sCD40L, IL-6 and oxLDL. Based on these results, sCD40L, IL-6, and oxLDL might be considered suitable markers for identifying patients at risk for cardiovascular events in addition to CRP and fibrinogen.

Results from meta-analyses indicate that the prognostic value of sCD40L, IL-6, and oxLDL for cardiovascular events is comparable to the prognostic 
value of CRP and fibrinogen. Danesh et al. estimated that the relative risk of CRP for coronary heart disease was 1.7 (95\% CI 1.4-2.1) [5]. This metaanalysis was updated for CRP in April 2004, in which the relative risk had decreased to 1.4 (95\% CI 1.3-1.7) [6]. For fibrinogen, Danesh reported a relative risk for coronary heart disease of 1.8 (95\% CI 1.6-2.0), whereas Ernst et al. reported a summarized relative risk of fibrinogen for cardiovascular events of 2.3 (95\% CI 1.9-2.8) [4, 5]. However, the results of our systematic review should be interpreted with consideration of our study limitations described below.

Two studies which were included in the review point to the possibility that combinations of marker concentrations may increase the prognostic value and will provide an even more detailed risk profile of the individual patient. For example, Varo et al. additionally investigated the prognostic value of the combined elevated concentrations of sCD40L and troponin for death, myocardial infarction and the combination death/myocardial infarction/chronic heart failure [20]. The adjusted relative risk estimates associated with elevated concentrations of both sCD40L and troponin were 12.1, 7.2 and 4.3, respectively $(\mathrm{p}<0.01$ for all). A similar analysis was performed by Luc et al. [31]. The population used in this study had an approximately 6-fold increased risk of myocardial infarction or coronary death when both IL-6 and LDL-cholesterol levels reached the highest tertile (as compared to patients with both IL-6 and LDL-cholesterol concentrations in the lowest tertile). Therefore, determining combinations of different marker concentrations might be more promising than determining single marker concentrations and in this respect sCD40L, IL-6, and oxLDL might be interesting candidates. However, additional studies have to be performed to corroborate this hypothesis.

Only two studies on IL-6 (Doo, Pai) [33, 34] presented a non-significant, adjusted relative risk estimate of 1.1 and 1.3, respectively. Doo et al. focussed mainly on the effect of antibodies against oxidized lipoproteins in patients with unstable angina pectoris [33]. The authors also determined levels of other markers, like CRP and cell adhesion molecules. However, since crude RR estimates were not presented, it is difficult to explain why the relative risk estimate in this study differed from all other studies on IL-6.

A limitation of the present review is that the number of sCD40L and oxLDL publications that fulfilled the inclusion criteria was scant. Only 4 and 2 publications, respectively, were selected. The small number of studies 
that investigate the prognostic value of soluble CD40 ligand in cardiovascular diseases can be explained by the fact that the association between high levels of sCD40L and cardiovascular disease has only been demonstrated recently. In 1999, Mach et al. demonstrated a possible link between CD40CD40L interaction and atherosclerosis, while in 2001 Aukrust et al. were the first to demonstrate the enhancement of soluble and membrane-bound CD40 ligand in patients with unstable angina pectoris [7,10]. Four years have passed since this publication. Therefore, only a small amount of prospective studies was to be expected. Future studies should give a more reliable indication of the true prognostic value of sCD40L levels in (cardiovascular) patients.

The number of articles for oxLDL was also low, while the association between oxLDL and cardiovascular disease has been known since the early 1990s. In the case of oxLDL, some studies were excluded because the marker concentration was not determined at baseline, or a relative risk estimate was not available. Because of the low number of studies on sCD40L and oxLDL, the problem of publication bias merits attention. Publication bias is a well-known problem in systematic reviews. Small studies with positive results tend to be accepted for publication more frequently than small studies with less favourable results. For this reason, it cannot be excluded that the prognostic value of the studied markers, especially sCD40L and oxLDL, was overestimated.

Another limitation is the heterogeneity of the included studies. Table 1 shows an overview of the variation in population size, follow-up period, study outcome, exposure categories and confounder adjustment. There was also a large variation in endpoints and/or combinations of endpoints, which made it impossible to evaluate the prognostic value of sCD40L, IL-6, and oxLDL for particular cardiovascular events. The large heterogeneity of the studies was the reason for refraining from pooling of the data from individual studies into one summary estimate.

Finally, it must be noted that only three studies adjusted for accepted serum markers, like CRP (Table 2). Therefore, this study does not demonstrate that $\mathrm{sCD} 40 \mathrm{~L}, \mathrm{IL}-6$, or oxLDL are strong predictors for the occurrence of cardiovascular events independent of other markers. Considering the limited number of longitudinal studies available, it is too early to draw definite conclusions. 
The results of this review suggest that the markers sCD40L, IL-6, and oxLDL have a prognostic value in predicting cardiovascular disease. Whether these presented markers can be used in everyday clinical practice remains to be determined. Other elements need to be additionally investigated before these markers might be introduced into the clinic. For example, it is not known if lowering concentrations of these markers will result in a more favourable prognosis for these patients. It is also important that diagnostic tests to determine marker concentration are readily available. This is not yet the case with respect to most serum markers, although more and more user friendly and commercially available diagnostic kits are becoming available. And last but not least, costs that are associated with screening for these markers would have to weigh up against the benefit gained from treating less patients with atherosclerosis-related complications.

In our opinion, the strengths of this review are fourfold: (1) it is the first study that summarizes the prognostic value of markers other than CRP and fibrinogen for cardiovascular disease in a systematic review, (2) it suggests that $\mathrm{SCD} 40 \mathrm{~L}, \mathrm{IL}-6$, and oxLDL might have a similar value in the prevention and treatment of atherosclerosis as CRP and fibrinogen, (3) it draws attention to the fact that future studies should be designed in a way that enables the pooling of data of several studies, and (4) it suggests that future investigators should not only examine the prognostic value of separate markers, but should additionally focus on determining the prognostic value for cardiovascular disease of combinations of different markers.

\section{CONCLUSIONS}

This systematic review showed that elevated concentrations of the serum markers sCD40L, IL-6, and oxLDL are associated with significantly increased risk of cardiovascular disease. However, there is a need for more longitudinal studies which evaluate their prognostic value adjusted for cardiovascular risk factors and accepted serum markers like CRP and fibrinogen. 


\section{REFERENCES}

1. Ross R. Atherosclerosis: an inflammatory disease. N Eng J Med. 1999; 340: 115-26.

2. Ridker PM, Buring JE, Shih J, Matias M, Hennekens CH. Prospective study of Creactive protein and the risk of future cardiovascular events among apparently healthy women. Circulation. 1998; 98: 731-3.

3. Yarnell JWG, Patterson CC, Sweetnam PM, Lowe GDO. Haemostatic/inflammatory markers predict 10 -year risk of IHD at least as well as lipids: the Caerphilly collaborative studies. European Heart Journal. 2004; 25: 1049-56.

4. Ernst E, Resch KL. Fibrinogen as a cardiovascular risk factor: a meta-analysis and review of the literature. Ann Intern Med. 1993; 118: 956-63.

5. Danesh J, Collins R, Appleby P, Peto R. Association of fibrinogen, C-reactive protein, albumin, or leukocyte count with coronary heart disease. JAMA. 1998; 279: 1477-82.

6. Danesh J, Wheeler JG, Hirschfield GM, Eda S, Eiriksdottir G, Rumley A, Lowe GD, Pepys MB, Gudnason V. C-reactive protein and other circulating markers of inflammation in the prediction of coronary heart disease. N Eng J Med. 2004; 350: 1387-97.

7. Mach F, Schönbeck U, Sukhova GK, Bourcier T, Bonnefoy JY, Pober JS, Libby P. Functional CD40 ligand is expressed on human vascular endothelial cells, smooth muscle cells, and macrophages: implications for CD40-CD40 ligand signalling in atherosclerosis. Proc Natl Acad Sci USA. 1997; 94: 1931-36.

8. Lutgens E, Daemen MJ. CD40-CD40L interactions in atherosclerosis. Trends Cardiovasc Med. 2002; 12: 27-32.

9. Lutgens E, Cleutjens KB, Heeneman S, Koteliansky VE, Burkly LC, Daemen MJ. Both early and delayed anti-CD40L antibody treatment induces a stable plaque phenotype. Proc Natl Acad Sci USA. 2000; 97: 7464-69.

10. Aukrust P, Müller F, Ueland T, Berget T, Aaser E, Brunsvig A, Solum NO, Forfang K, Frøland SS, Gullestad L. Enhanced levels of soluble and membrane-bound CD40 ligand in patients with unstable angina. Possible reflection of $\mathrm{T}$ lymphocyte and platelet involvement in the pathogenesis of acute coronary syndromes. Circulation. 2001; 100: 614-20.

11. Biasucci LM, Vitelli A, Liuzzo G, Altamura S, Caligiuri G, Monaco C, Rebuzzi AG, Ciliberto G, Maseri A. Elevated levels of interleukin-6 in unstable angina. Circulation. 1996; 94: 874-77.

12. Rifai N, Joubran R, Yu H, Asmi M, Jouma M. Inflammatory markers in men with angiographically documented coronary heart disease. Clinical Chemistry. 1999; 45: 1967 73.

13. Maier W, Altwegg LA, Corti R, Gay S, Hersberger M, Maly FE, Sütsch G, Roffi M, Neidhart M, Eberli FR, Tanner FC, Gobbi S, von Eckardstein A, Lüscher TF. Inflammatory markers at the site of ruptured plaque in acute myocardial infarction. Locally increased interleukin- 6 and serum amyloid A but decreased C-reactive protein. Circulation. 2005; 111: 1355-61.

14. Schieffer B, Selle T, Hilfiker A, Hilfiker-Kleiner D, Grote K, Tietge UJ, Trautwein C, Luchtefeld M, Schmittkamp C, Heeneman S, Daemen MJ, Drexler H. Impact of interleukin-6 on plaque development and morphology in experimental atherosclerosis. Circulation. 2004; 110: 3493-3500. 
15. Ehara S, Ueda M, Naruko T, Haze K, Itoh A, Otsuka M, Komatsu R, Matsuo T, Itabe H, Takano T, Tsukamoto Y, Yoshiyama M, Takeuchi K, Yoshikawa J, Becker AE. Elevated levels of oxidized low density lipoprotein show a positive relationship with the severity of acute coronary syndromes. Circulation. 2001; 103: 1955-60.

16. Toshima S, Hasegawa A, Kurabayashi M, Itabe H, Takano T, Sugano J, Shimamura K, Kimura J, Michishita I, Suzuki T, Nagai R. Circulating oxidized low density lipoprotein levels. A biochemical risk marker for coronary heart disease. Arterioscler Thromb Vasc Biol. 2000; 20: 2243-47.

17. Weinbrenner T, Cladellas M, Isabel Covas M, Fitó M, Tomás M, Sentí M, Bruguera J, Marrugat J. High oxidative stress in patients with stable coronary heart disease. Atherosclerosis. 2003; 168: 99-106.

18. Schönbeck U, Varo N, Libby P, Buring J, Ridker PM. Soluble CD40 ligand and cardiovascular risk in women. Circulation. 2001; 104: 2266-68.

19. Baldus S, Heeschen C, Meinertz T, Zeiher AM, Eiserich JP, Münzel T, Simoons ML, Hamm CW, CAPTURE Investigators. Myeloperoxidase serum levels predict risk in patients with acute coronary syndromes. Circulation. 2003; 108: 1440-45.

20. Varo N, de Lemos JA, Libby P, Morrow DA, Murphy SA, Nuzzo R, Gibson CM, Cannon CP, Braunwald E, Schönbeck Ul. Soluble CD40L: risk prediction after acute coronary syndromes. Circulation. 2003; 108: 1049-52.

21. Heeschen C, Dimmeler S, Hamm CW, van den Brand MJ, Boersma E, Zeiher AM, Simoons ML, CAPTURE Study Investigators. Soluble CD40 ligand in acute coronary syndromes. N Engl J Med. 2003; 348: 1104-11.

22. Kinlay S, Schwartz GG, Olsson AG, Rifai N, Sasiela WJ, Szarek M, Ganz P, Libby P; Myocardial Ischemia Reduction with Aggressive Cholesterol Lowering (MIRACL) Study Investigators. Effect of atorvastatin on risk of recurrent cardiovascular events after an acute coronary syndrome associated with high soluble CD40 ligand in the Myocardial Ischemia Reduction with Aggressive Cholesterol Lowering (MIRACL) Study. Circulation. 2004; 110: 386-91.

23. Heeschen C, Dimmeler S, Hamm CW, Fichtlscherer S, Simoons ML, Zeiher AM; CAPTURE Study Investigators. Pregnancy-associated plasma protein-A levels in patients with acute coronary syndromes. J Am Coll Cardiol. 2005; 45: 229-37.

24. Harris TB, Ferrucci L, Tracy RP, Corti MC, Wacholder S, Ettinger WH Jr, Heimovitz $\mathrm{H}$, Cohen HJ, Wallace R. Associations of elevated interleukin- 6 and C-reactive protein levels with mortality in elderly. Am J Med. 1999; 106: 506-12.

25. Ridker PM, Rifai N, Stampfer MJ, Hennekens CH. Plasma concentration of interleukin6 and the risk of future myocardial infarction among apparently healthy men. Circulation. 2000; 101: 1767-72.

26. Ridker PM, Hennekens CH, Buring JE, Rifai N. C-reactive protein and other markers of inflammation in the prediction of cardiovascular disease in women. $\mathrm{N}$ Engl J Med. 2000; 342: 836-43.

27. Koukkunen H, Penttilä K, Kemppainen A, Halinen M, Penttila I, Rantanen T, Pyörälä K.. C-reactive protein, fibrinogen, interleukin- 6 and tumour necrosis factor-alpha in the prognostic classification of unstable angina pectoris. Ann Med. 2001; 33: 37-47.

28. Lindmark E, Diderholm E, Wallentin L, Siegbahn A. Relationship between interleukin-6 and mortality in patients with unstable coronary artery disease: effects of an early invasive or non-invasive strategy. JAMA. 2001; 286: 2107-13.

29. Volpato S, Guralnik JM, Ferrucci L, Balfour J, Chaves P, Fried LP, Harris TB. Cardiovascular disease, interleukin- 6 , and risk of mortality in older women: the women's health and aging study. Circulation. 2001; 103: 947-53. 
30. Pradhan AD, Manson JE, Rossouw JE, Siscovick DS, Mouton CP, Rifai N, Wallace RB, Jackson RD, Pettinger MB, Ridker PM. Inflammatory biomarkers, hormon replacement therapy, and incident coronary heart disease: prospective analysis from the Women's Health Initiative observational study. JAMA. 2002; 288: 980-87.

31. Luc G, Bard JM, Juhan-Vague I, Ferrieres J, Evans A, Amouyel P, Arveiler D, Fruchart JC, Ducimetiere P, PRIME Study Group. C-reactive protein, interleukin-6, and fibrinogen as predictors of coronary heart disease: the PRIME Study. Arterioscler Thromb Vasc Biol. 2003; 23: 1255-61.

32. Conway DSG, Buggins P, Hughes E. Prognostic significance of raised plasma levels of interleukin-6 and C-reactive protein in atrial fibrillation. Am Heart J. 2004; 148: 462-66.

33. Doo YC, Han SJ, Lee JH, Cho GY, Hong KS, Han KR, Lee NH, Oh DJ, Ryu KH, Rhim CY, Lee KH, Lee Y. Associations among oxidized low-density lipoprotein antibody, C-reactive protein, interleukin- 6 , and circulating cell adhesion molecules in patients with unstable angina pectoris. Am J Cardiol. 2004; 93: 554-58.

34. Pai JK, Pischon T, Ma J, Manson JE, Hankinson SE, Joshipura K, Curhan GC, Rifai N, Cannuscio CC, Stampfer MJ, Rimm EB. Inflammatory markers and the risk of coronary heart disease in men and women. N Engl J Med. 2004; 351: 2599-2610.

35. Holvoet P, Harris TB, Tracy RP, Verhamme P, Newman AB, Rubin SM, Simonsick EM, Colbert LH, Kritchevsky SB. Association of high coronary heart disease risk status with circulating oxidized LDL in the well-functioning elderly. Findings from the Health, Aging and Body Composition Study. Arterioscler Thromb Vasc Biol. 2003; 23: 1444-48.

36. Shimada K, Mokuno H, Matsunaga E, Miyazaki T, Sumiyoshi K, Miyauchi K, Daida H. Circulating oxidized low-density lipoprotein is an independent predictor for cardiac event in patients with coronary artery disease. Atherosclerosis. 2004; 174: 343-47.

37. Holvoet P, Kritchevsky SB, Tracy RP, Mertens A, Rubin SM, Butler J, Goodpaster B, Harris TB. The metabolic syndrome, circulating oxidized LDL, and risk of myocardial infarction in well-functioning elderly people in the health, aging and body composition cohort. Diabetes. 2004; 53: 1068-73.

\section{ACKNOWLEDGEMENTS}

S.H. and M.D. participate in the European Vascular Genomics Network (http://www.evgn.org), a Network of Excellence supported by the European Community's Sixth Framework Program for Research Priority 1 (Life Sciences, Genomics, and Biotechnology for Health; contract LSHM-CT2003-503254). This work was supported by the Dutch Heart Foundation, grant number 2002.B033, and by the 'Besluit Subsidies Investeringen Kennisinfrastructuur' (BSIK) program entitled 'Molecular imaging of ischemic heart disease (project number BSIK 03033). 



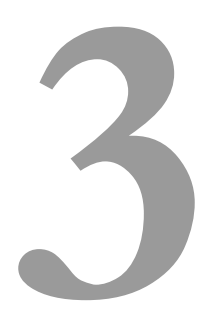

Leukocyte counts, myeloperoxidase, and pregnancy-associated plasma protein $\mathrm{A}$ as biomarkers for cardiovascular disease: old and new markers evalued

M.B.I. Lobbes, M.E. Kooi, E. Lutgens, A.W. Ruiters, V. Lima Passos, S.H.J.G. Braat, M. Rousch, H. Ten Cate, J.M.A. van Engelshoven, M.J.A.P. Daemen, S. Heeneman 


\section{ABSTRACT}

Background - Cardiovascular disease is associated with elevated serum concentrations of biomarkers. Established biomarkers for cardiovascular endpoints are leukocyte counts, fibrinogen, and CRP, whereas upcoming biomarkers are MPO and PAPP-A. For these novel biomarkers, only few studies have been reported, creating a need for further validation. Most studies focus on acute coronary syndromes (e.g. unstable angina pectoris or myocardial infarction), without evaluating the association in less acute stages of the disease (e.g. stable angina pectoris).

Materials and methods - We evaluated leukocyte counts and levels of CRP, fibrinogen, MPO, and PAPP-A in patients with stable and unstable angina pectoris, acute myocardial infarction, and healthy controls (30 patients per group). All biomarkers were analyzed again after 6 months.

Results - Compared to controls, blood leukocyte counts and serum concentrations of fibrinogen, CRP, MPO, and PAPP-A were significantly increased in patients with acute myocardial infarction. Leukocyte counts and concentrations of MPO were significantly increased in patients with unstable angina pectoris compared with controls. After 6 months, blood leukocyte counts and MPO serum concentrations were still increased in patients with acute myocardial infarction when compared to controls. When combined, leukocyte counts, MPO and PAPP-A classified study group designation for acute coronary events correctly in $82 \%$ of the cases.

Conclusion - Leukocyte counts and levels of fibrinogen, CRP, PAPP-A and MPO were increased in patients with acute coronary syndromes. After 6 months, leukocyte counts and MPO concentrations were still increased in patients with acute myocardial infarction when compared to controls. 


\section{INTRODUCTION}

Atherosclerosis is considered an inflammatory disease of the large arteries that is characterized by the formation of atherosclerotic plaques. In the majority of cases clinical events of atherosclerosis, like myocardial infarction or ischemic stroke, are caused by rupture of an atherosclerotic lesion [1, 2].

Several inflammatory molecules have been put forward as biomarkers for plaque vulnerability. Biomarkers are biochemical features that can be used to measure the disease progress or the effect of treatment [3]. In the context of atherosclerosis, concentrations of CRP and fibrinogen, and the count of leukocytes in blood have been investigated most extensively [4-6]. However, large meta-analyses have demonstrated that their prognostic value for assessing risk of cardiovascular disease or adverse outcomes is limited [6-9]. Therefore, there is a continuous search for novel, more powerful biomarkers that are able to predict the occurrence of future cardiovascular complications.

In this respect, MPO and PAPP-A have been suggested as potential biomarkers for cardiovascular disease. However, publications on these biomarkers are still scant and their predictive value still needs to be assessed. Also, these studies focus mainly on acute coronary syndromes and lack follow-up information [3]. Therefore, our aim was to assess the association of MPO and PAPP-A in several groups of patients with cardiovascular disease and compare them with more established biomarkers of cardiovascular disease, like leukocyte counts and concentrations of fibrinogen and CRP. In addition, we investigated the presence of these five biomarkers after 6 months follow-up.

\section{MATERIALS AND METHODS}

For this study, we included 30 patients per group since power analysis based on available literature data, a $95 \%$ confidence interval and a power of $90 \%$ indicated that a minimum of 25 patients per group would be needed to obtain sufficient significance. Therefore, the total study cohort consisted of 120 patients, who were divided into four groups: stable angina pectoris (SAP), unstable angina pectoris (UAP), acute myocardial infarction (AMI) and controls $(\mathrm{CON})$. Venous blood samples were drawn from all participants at inclusion and after 6 months follow-up. Also, a standardized questionnaire regarding patient characteristics and follow-up outcome was pre- 
sented at study inclusion and follow-up. The study protocol was approved by the institutional medical ethics committee. All patients provided written, informed consent.

Patients with SAP that were scheduled for a percutaneous coronary intervention (PCI) were recruited from the outpatient clinic. Only patients with more than $50 \%$ stenosis of one or more of the main coronary branches (as proven by coronary angiography) were included. Evaluation of the coronary stenosis was performed by cardiologists blinded for study group. Patients with UAP presented themselves with prolonged new-onset chest pain $(<30$ days), an accelerating pattern of chest pains or with chest pains occurring at lesser degrees of exertion or at rest. UAP was characterized by ischemic ECG changes (such as ST segment elevation, reciprocal ST segment depression, $\mathrm{T}$ wave inversion or development of $\mathrm{Q}$ waves) without elevation of cardiac enzymes (such as troponin-T, creatine kinase and lactate dehydrogenase isozymes) or by elevation of cardiac enzymes without apparent ischemic ECG changes. Patients with AMI presented themselves with an acute onset of chest pain, ischemic ECG changes, and elevation of cardiac enzymes and troponin T. Diagnoses of UAP or AMI were made by cardiologists blinded for the study group. The control group consisted of individuals of the general population older than 45 years, but free of cardiovascular disease and serious illnesses for the past 6 months.

In all groups, exclusion criteria were: inability to provide informed consent, recent acute coronary event, stroke or transient ischemic attack (all events less than 6 months prior to study inclusion), history of resuscitation or cardiogenic shock, renal insufficiency (creatinin clearance $<40 \mathrm{~mL} / \mathrm{min}$ according to the Cockroft formula), current inflammatory disease, auto-immune diseases, and the presence of a disorder with a high chance of death within 5 years (e.g. malignancies).

Blood samples were drawn from the antecubal vein. In patients with SAP, blood sampling was performed immediately prior to percutaneous coronary intervention. In patients with UAP and AMI, blood was drawn and processed within 12 hours after the last ischemic episode and before intervention. Levels of fibrinogen, total cholesterol, HDL, LDL, CK, creatinine, and leukocyte counts were determined with standard hospital laboratory assays. The blood samples used for the determination of CRP, PAPP-A, and MPO were centrifuged immediately after sampling and frozen at $-80^{\circ} \mathrm{C}$ until assays 
were performed. All samples were thawed only once. All laboratory analyses were performed blinded.

Enzyme-linked immunosorbent assays were performed to determine serum levels of CRP (Kordia, Leiden, The Netherlands), PAPP-A, and MPO (all DRG Instruments $\mathrm{GmbH}$, Germany). In addition, a new ultrasensitive assay kit for PAPP-A was used. This was a research kit from DRG Instruments, which is not available in the USA. It uses a monoclonal antibody specific for patients with acute myocardial infarction, whereas the regular kit uses a polyclonal antibody. Assays were performed according to the instructions provided by the manufacturer. Assay ranges were $1-25 \mathrm{mg} / \mathrm{l}$ for CRP, $0-30 \mu \mathrm{g} / \mathrm{l}$ for PAPP-A, and $0-450 \mu \mathrm{g} / 1$ for ultra-sensitive (US) PAPP-A. For MPO, the detection limit was $<3 \mu \mathrm{g} / \mathrm{l}$.

\section{Statistical analysis}

Statistical analysis was carried out with SPSS 15.0 (Chicago, USA). Since the distribution of biomarker levels was slightly skewed, statistical tests were performed on logarithmically transformed biomarker values, while mean (arithmetic) values of these values were presented. Depending on the research questions and the outcome variables, three different tests were applied. First, a univariate ANOVA was used to determine which variables were significantly associated with the biomarkers baseline measurements. Multicollinearity among explanatory variables (see also Table 1) was checked via the variance inflation factor and a top-down procedure was used to determine the final model parameters. For all models the group's effect on the individual biomarkers was adjusted for sex, smoking, family history of cardiovascular disease, daily aspirin use, and exercise (defined as a significant increase in heart rate for more than 30 minutes per week). Additional adjustment for age and renal clearance was performed in the analysis of fibrinogen and PAPP-A concentrations, and for body mass index when the outcome was CRP. Adjusted posthoc groups comparisons, were carried out using the Bonferroni correction. Second, a repeated measures ANOVA was used in order to evaluate changes in biomarker levels in time with groups as a fixed between-subjects factor. Third, a stepwise discriminant analysis was performed to assess which biomarkers could best discriminate between the four study groups. All p-values $<0.05$ were considered statistically significant. 


\section{RESULTS}

General patient characteristics are presented in Table 1. Gender, smoking, exercise, family history for cardiovascular disease, the use of beta-blockers, aspirin and statins, creatine kinase, troponin T, cholesterol and LDL-C levels, and diastolic blood pressure differed significantly between study groups.

At study inclusion, leukocyte counts differed significantly between controls and patients with AMI or UAP (both $\mathrm{p}<0.001$ ). There was no significant difference between controls and patients with SAP. In addition, leukocyte counts were significantly higher in patients with AMI than in patients with UAP $(p=0.002)$ or SAP $(p<0.001$, Figure 1 and Table 2$)$. There was a significant decrease in leukocyte counts for patients with UAP or AMI after 6 months ( $\mathrm{p}=0.011$ and $\mathrm{p}<0.001$, respectively), while there were no significant changes in leukocyte counts in patients with SAP or controls. A significantly increased leukocyte count between AMI and CON remained $(p=0.002)$, whereas all other pairwise comparisons were non-significant (Figure 2).

For fibrinogen, serum concentrations were significantly higher in patients with AMI compared with controls $(\mathrm{p}=0.02)$. There was no significant difference between CON and UAP or SAP, or between AMI and UAP, or between UAP and SAP. However, there was a trend for a difference between AMI and SAP ( $p=0.07$, Figure 1 and Table 2). After 6 months follow-up, there was only a significant decrease in fibrinogen concentration in patients with AMI $(\mathrm{p}=0.034)$. There were no significant differences in fibrinogen concentrations between groups at this time point (Figure 2).

For CRP, there was a significantly higher concentration in patients with AMI compared with controls $(\mathrm{p}=0.02)$. There was no significant difference between controls and patients with UAP or SAP, and no significant differences between AMI and SAP or UAP, or between UAP and SAP (Figure 1, Table 2). At follow-up, CRP concentrations were lower in patients with $\operatorname{UAP}(p=0.009)$ or AMI $(p=0.009)$, and CON ( $p=0.016)$. At this time point, there were no significant differences in CRP concentrations between groups (Figure 2). 
Table 1. Baseline patient characteristics per study group. Exercise is defined as a significant rise in heart rate for more than 30 minutes and at least once a week. Data are presented as mean \pm standard deviation $\left(^{*}\right)$ and as percentage of the total number of study group participants $\left({ }^{* *}\right)$. Abbrevations are: units (U), international units (IU), cardiovascular disease (CVD), not significant (NS), and blood pressure (BP).

\begin{tabular}{|c|c|c|c|c|c|}
\hline Characteristics & SAP & UAP & AMI & $\mathrm{CON}$ & $\mathrm{p}$-value \\
\hline Total participants & 30 & 30 & 30 & 30 & \\
\hline Age (years)* & $63.6(10.5)$ & $65.3(13.0)$ & $59.5(12.6)$ & $65.0(9.5)$ & NS \\
\hline Male $(\%)^{* *}$ & $28(93.3)$ & $16(55.1)$ & $24(80.0)$ & $19(63.3)$ & 0.004 \\
\hline Smoking $(\%)^{* *}$ & $7(23.3)$ & $5(16.7)$ & $13(43.3)$ & $4(13.3)$ & 0.034 \\
\hline Exercise $(\%)^{* *}$ & $14(46.7)$ & $15(50.0)$ & $8(26.7)$ & $22(73.3)$ & 0.004 \\
\hline Alcohol (U/day)* & $0.7(1.3)$ & $0.4(0.7)$ & $0.5(0.9)$ & $1.3(1.1)$ & 0.064 \\
\hline Family history CVD (\%)** & $26(86.7)$ & $18(60.0)$ & $14(46.7)$ & $19(63.3)$ & 0.013 \\
\hline Diabetes $(\%)^{* *}$ & $3(10.0)$ & $7(23.3)$ & $1(3.3)$ & $3(10.0)$ & NS \\
\hline Beta-blocker use $(\%)^{* *}$ & $30(100.0)$ & $15(50.0)$ & $12(40.0)$ & $5(16.7)$ & $<0.001$ \\
\hline Statin use $(\%)^{* *}$ & $26(86.7)$ & $18(60.0)$ & $12(40.0)$ & $6(20.0)$ & $<0.001$ \\
\hline Aspirin use $(\%)^{* *}$ & $13(43.3)$ & $14(46.7)$ & $12(40.0)$ & $1(3.3)$ & 0.001 \\
\hline Anti-diabetica use $(\%)^{* *}$ & $2(6.7)$ & $5(16.7)$ & $1(3.3)$ & $3(10.0)$ & NS \\
\hline Cholesterol $(\mathrm{mmol} / \mathrm{L})^{*}$ & $4.2(1.0)$ & $4.7(1.3)$ & $5.5(1.1)$ & $5.6(1.3)$ & $<0.001$ \\
\hline $\mathrm{HDL}(\mathrm{mmol} / \mathrm{L})^{*}$ & $1.1(0.2)$ & $1.0(0.2)$ & $1.0(0.2)$ & $1.2(0.4)$ & NS \\
\hline $\mathrm{LDL}(\mathrm{mmol} / \mathrm{L})^{*}$ & $2.1(1.0)$ & $3.0(1.1)$ & $3.9(1.0)$ & $3.6(1.1)$ & $<0.001$ \\
\hline AST (IU/L)* & $26(13)$ & $30(16)$ & $100(140)$ & $22(11)$ & NS \\
\hline $\operatorname{ALT}(\mathrm{IU} / \mathrm{L})^{*}$ & $34(17)$ & $31(19)$ & $37(22)$ & $36(29)$ & NS \\
\hline Renal clearance $(\mathrm{mL} / \mathrm{min}) *$ & $85.8(29.7)$ & $83.7(28.2)$ & $93.2(20.1)$ & $83.7(26.4)$ & NS \\
\hline Systolic BP $(\mathrm{mmHg})^{*}$ & $131.0(14.8)$ & $141.3(22.0)$ & $129.7(30.0)$ & $132.2(15.6)$ & NS \\
\hline Diastolic BP (mmHg)* & $76.6(7.9)$ & $77.5(15.3)$ & $73.8(15.4)$ & $82.8(8.6)$ & 0.001 \\
\hline Body mass index $\left(\mathrm{kg} / \mathrm{m}^{2}\right)^{*}$ & $26.4(3.7)$ & $25.8(2.9)$ & $27.1(3.5)$ & $26.4(3.9)$ & NS \\
\hline Troponin-T $(\mu \mathrm{g} / \mathrm{L})^{*}$ & $0.00(0.00)$ & $0.21(0.13)$ & $2.08(0.73)$ & $0.00(0.00)$ & 0.003 \\
\hline Creatin kinase $(\mathrm{U} / \mathrm{L})^{*}$ & $154.0(107.5)$ & $148.3(141.3)$ & 639.6 (913.8) & $116.1(47.4)$ & $<0.001$ \\
\hline
\end{tabular}

For MPO, serum concentrations were significantly higher in patients with UAP $(p<0.001)$ or AMI $(p<0.001)$ compared with controls. There was no significant difference in serum MPO concentrations between patients with SAP or controls. Furthermore, serum MPO levels were significantly higher in AMI and UAP compared with SAP (both $\mathrm{p}<0.001$ ), but there was no difference between AMI and UAP (Figure 1 and Table 2). At follow-up, the mean MPO concentrations had significantly decreased in patients with SAP $(p=0.008)$, UAP $(p<0.001)$, or AMI $(p<0.001)$ and CON $(p<0.001)$. At 6 months, MPO concentrations in patients with AMI were still increased when compared with CON (Figure 2, $\mathrm{p}=0.046$ ). 
Table 2. Pairwise comparisons of serum biomarker level differences between study groups. Abbrevations: not significant (NS).

\begin{tabular}{|c|c|c|c|c|c|c|c|}
\hline \multicolumn{4}{|c|}{ Leucocyte counts (billions/L) } & \multicolumn{4}{|c|}{ Fibrinogen $(\mathrm{g} / \mathrm{L})$} \\
\hline Group & Comp. & Mean diff. & $\mathrm{p}$-value & Group & Comp. & Mean diff. & p-value \\
\hline \multirow[t]{3}{*}{$\mathrm{CON}$} & SAP & 0.246 & NS & $\mathrm{CON}$ & SAP & 0.052 & NS \\
\hline & UAP & 2.485 & $<0.001$ & & UAP & 0.236 & NS \\
\hline & AMI & 4.980 & $<0.001$ & & AMI & 0.712 & 0.02 \\
\hline \multirow[t]{2}{*}{ AMI } & SAP & 4.733 & $<0.001$ & AMI & SAP & 0.764 & NS \\
\hline & UAP & 2.495 & 0.002 & & UAP & 0.476 & NS \\
\hline UAP & SAP & 2.239 & $<0.001$ & UAP & SAP & 0.288 & NS \\
\hline \multicolumn{4}{|c|}{ C-reactive protein $(\mathrm{mg} / \mathrm{L})$} & \multicolumn{4}{|c|}{ Myeloperoxidase ( $\mu \mathrm{g} / \mathrm{L})$} \\
\hline Group & Comp. & Mean diff. & p-value & Group & Comp. & Mean diff. & p-value \\
\hline \multirow[t]{3}{*}{$\mathrm{CON}$} & SAP & 1.428 & NS & $\mathrm{CON}$ & SAP & 86.59 & NS \\
\hline & UAP & 1.408 & NS & & UAP & 986.55 & $<0.001$ \\
\hline & AMI & 9.505 & 0.02 & & AMI & 1375.86 & $<0.001$ \\
\hline \multirow[t]{2}{*}{ AMI } & SAP & 8.077 & NS & AMI & SAP & 1462.44 & $<0.001$ \\
\hline & UAP & 8.097 & NS & & UAP & 398.31 & NS \\
\hline UAP & SAP & 0.020 & NS & UAP & SAP & 1073.14 & $<0.001$ \\
\hline \multicolumn{4}{|c|}{ PAPP-A (mg/L) } & \multicolumn{4}{|c|}{ US PAPP-A (ng/mL) } \\
\hline Group & Comp. & Mean diff. & $\mathrm{p}$-value & Group & Comp. & Mean diff. & p-value \\
\hline \multirow[t]{3}{*}{$\mathrm{CON}$} & SAP & 0.080 & NS & $\mathrm{CON}$ & SAP & 1.288 & NS \\
\hline & UAP & 0.187 & NS & & UAP & 2.123 & NS \\
\hline & AMI & 0.129 & NS & & AMI & 25.527 & 0.001 \\
\hline \multirow[t]{2}{*}{ AMI } & SAP & 0.050 & NS & AMI & SAP & 32.885 & 0.001 \\
\hline & UAP & 0.060 & NS & & UAP & 54.200 & 0.001 \\
\hline UAP & SAP & 0.110 & NS & UAP & SAP & 1.648 & NS \\
\hline
\end{tabular}

Finally, for PAPP-A, no significant differences were demonstrated between the controls and the three groups of cardiovascular disease for the regular assay kit (Figure 1, Table 2). Based on these results, we decided not to analyze serum PAPP-A concentrations in the 6 months follow-up blood samples with the regular assay kit. In contrast, the US PAPP-A kit showed a significantly higher concentration of PAPP-A in AMI when compared with the other study groups (all $\mathrm{p}=0.001$ ). After 6 months, no significant differences in PAPP-A concentrations (as determined by the research kit) were observed (Figure 2).

A stepwise discriminant analysis was performed to investigate which combination of the five biomarkers could differentiate between the four groups 
at study inclusion. In the stepwise procedure, only leukocyte counts, MPO, and PAPP-A US emerged as having significant discriminative power. Based on linear combinations of these markers, subjects could be properly allocated to their specific groups in $61.6 \%(58.6 \%$ in the leave-one-out crossvalidation) of the cases (Table 3). Visual inspection of the discriminant functions' territory map revealed that the centroids of SAP and CON almost overlapped, suggesting similarity of profiles as regards their biomarkers' distributions. This motivated three subsequent analyses. In the first one, SAP and CON were combined in one group. With this new subdivision (i.e. three diagnostic categories) patients' correct classification improved to $82.8 \%$ (cross-validation 80.8\%), based on the same three biomarkers (Table $3)$.

Table 3. Results of different discriminant analyses.

\begin{tabular}{lll}
\hline Groups (total number) & Significant biomarkers & $\begin{array}{l}\text { Correct } \\
\text { classification }\end{array}$ \\
\hline CON-SAP-UAP-AMI (4) & Leukocyte counts, MPO, PAPP-A US & $61.6 \%$ \\
CON/SAP-UAP-AMI (3) & Leukocyte counts, MPO, PAPP-A US & $82.8 \%$ \\
CON/SAP-UAP/AMI (2) & MPO, PAPP-A US & $89.3 \%$ \\
UAP-AMI (2) & Leukocyte counts, PAPP-A US & $82.4 \%$ \\
\hline
\end{tabular}

In the second analysis, a broader dichotomous categorisation of patients in non-acute coronary events/controls (SAP and CON) versus acute coronary events (UAP and AMI) was considered. Here, patients' classification by leukocyte counts and MPO was correct in $89.3 \%$ of the cases (crossvalidation $87.5 \%$, Table 3). However, PAPP-A US had no further contribution and the discriminant functions were based solely on leucocyte counts and MPO. In contrast, PAPP-A US and leukocyte counts were the only markers selected in the third analysis, in which discriminant functions were required to separate between AMI and UAP patients. In this last analysis $82.4 \%$ of the acute patients were correctly assigned to their corresponding groups (cross-validation $80.4 \%$, Table 3 ). 

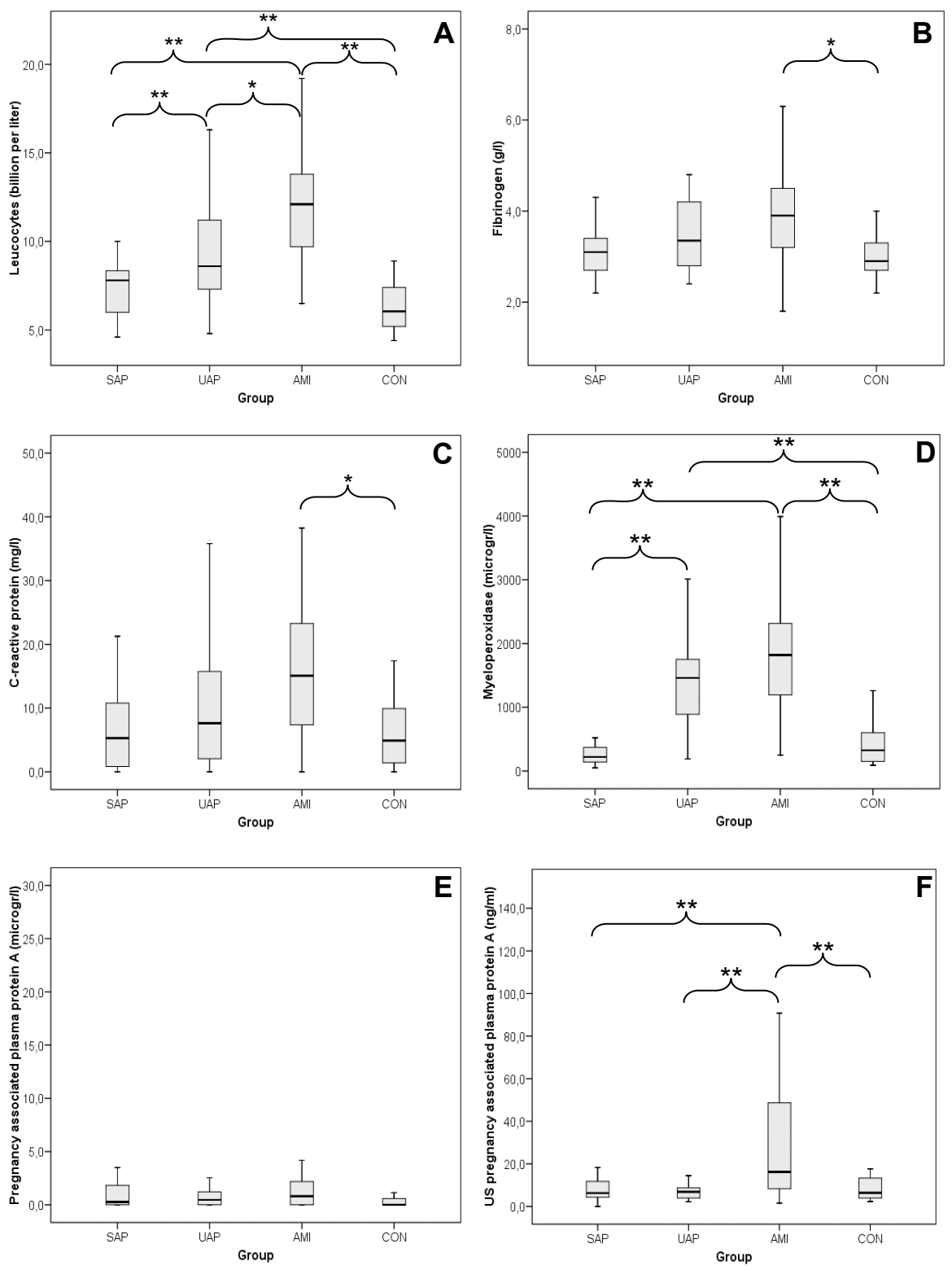

Figure 1. Boxplots of biomarker levels at study inclusion of leukocyte counts (A), fibrinogen (B), CRP (C), MPO (D), PAPP-A (E), and US PAPP-A (F). Statistical differences are expressed as $\mathrm{p}<0.05(*)$ or $\mathrm{p} \leq 0.01(* *)$. 

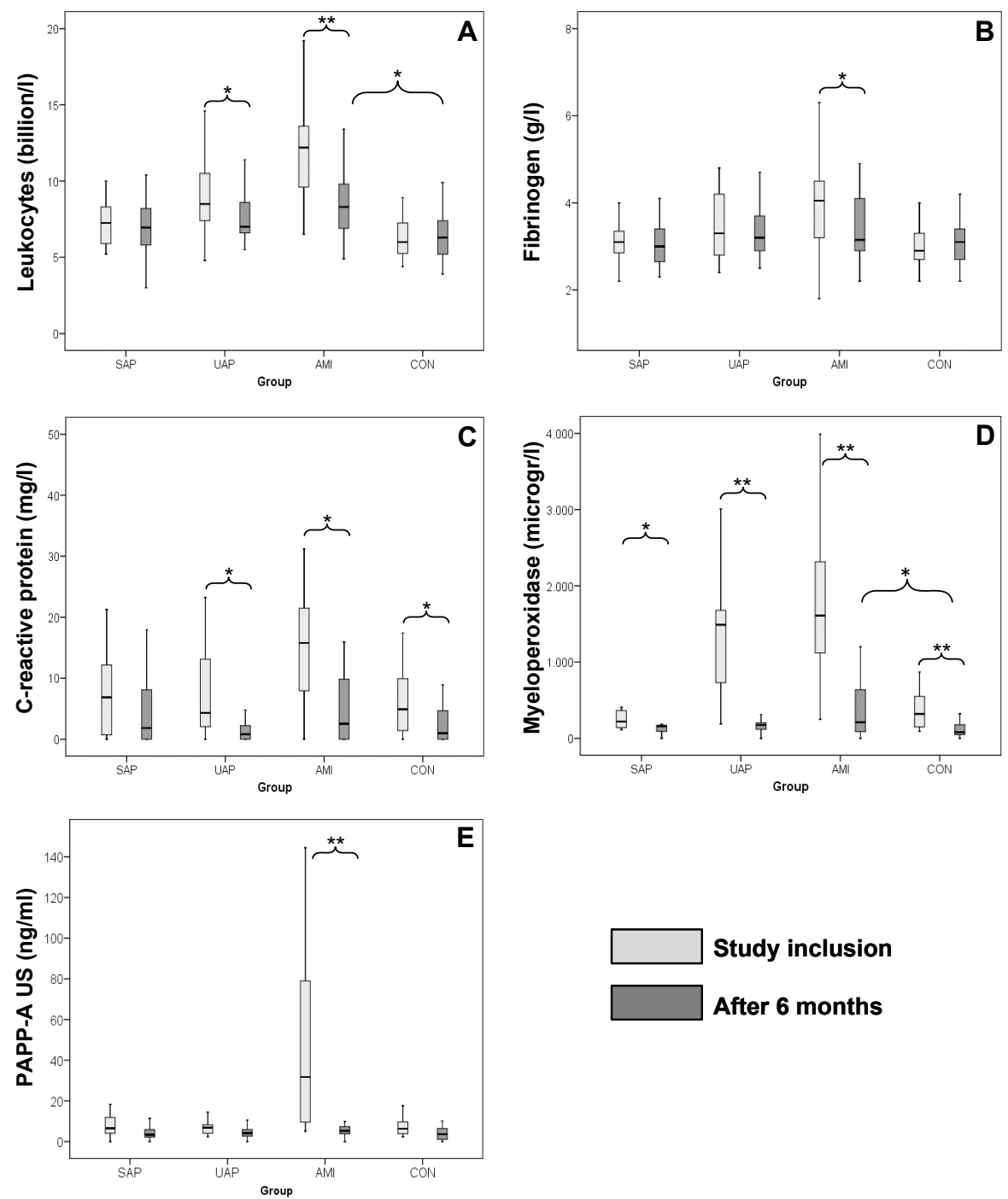

Study inclusion

After 6 months

Figure 2. Boxplots of biomarker levels of leukocyte counts (A), fibrinogen (B), CRP (C), MPO (D), and PAPP-A (E) at study inclusion and after 6 months follow-up. Statistical differences are expressed as $\mathrm{p}<0.05\left(^{*}\right)$ or $\mathrm{p} \leq 0.001(* *)$. 


\section{DISCUSSION}

In the current study, leukocyte counts were significantly increased in patients with AMI or UAP when compared with controls, while leukocyte counts in SAP were comparable to healthy controls. Significantly increased levels of fibrinogen and CRP were found in patients with AMI when compared with controls. The MPO concentrations were significantly increased in patients with AMI and UAP when compared with controls, In addition, concentrations of PAPP-A were significantly higher in AMI when compared with other study groups, but only if the ultrasensitive PAPP-A ELISA kit was used. These results showed that inflammatory markers were increased in patients with acute coronary events (especially myocardial infarction) and are in line with available literature, which also showed an increase of these markers after acute coronary events $[8,10,11]$.

Many studies have highlighted the predictive value of CRP for cardiovascular events. However, large meta-analyses showed only moderate prognostic value (relative risk estimates in the range of 1.3 to 1.5 ) $[6,8,11,12]$. Similar results have been published on the use of fibrinogen concentrations and leukocyte counts for the prediction of cardiovascular events and outcome (relative risk estimates of 1.4 to 1.8 for fibrinogen and approximately 1.4 for leukocyte counts) [6]. This creates a need for biomarkers for cardiovascular disease with an increased predictive value. In addition, the use of a panel of several biomarkers might even further increase the predictive value for cardiovascular events. With this respect, MPO and PAPP-A have been suggested as potential novel biomarkers for cardiovascular disease [10,13].

MPO has been associated with cardiovascular disease in many studies. Zhang et al. showed that elevated serum levels of MPO were associated with coronary artery disease, as visualized by angiography [14]. Additional studies demonstrated an increased cardiac risk with elevated serum levels of MPO in patients with acute coronary syndromes, and an increased odds ratio for major adverse cardiac events in a cohort of patients presenting with chest pains $[10,15]$. More recently, above median plasma MPO concentrations of AMI patients were associated with an increased risk for mortality [16]. In patients with chest pain, MPO proved to be an independent predictor of AMI on long-term follow-up [17]. These results are in agreement with our observations, since we found a significant increase in serum MPO levels in patients with UAP or AMI, when compared with healthy controls. We did not find any significant difference in MPO levels between controls and 
patients with SAP, which is in contrast by data published by Ruef et al., that showed an increased concentration of plasma MPO levels for patients with SAP or acute coronary syndromes [18]. However, direct comparison of MPO levels between studies remains difficult, because many studies differ in their blood sample collection (plasma or serum) and their laboratory assays to determine MPO concentrations.

Positive immunohistochemical staining for PAPP-A in advanced atherosclerotic plaques and an elevation of serum PAPP-A levels in acute coronary syndromes was shown by Bayes-Genis et al. [13]. PAPP-A is an independent predictor of adverse outcome and elevated levels were associated with an increased risk for death or myocardial infarction in patients with acute coronary syndromes $[19,20]$. Interestingly, Dominguez-Rodriguez et al., applying the regular PAPP-A kit as used in the present study (DRG International), did not find any significant differences in PAPP-A concentrations between patient with AMI and controls, and no association between PAPPA levels and risk of AMI [21]. These latter results are in agreement with our findings when using the regular PAPP-A assay kit with which we also did not find any significant differences in PAPP-A levels between patients with SAP, UAP, AMI, and controls. However, the ultrasensitive PAPP-A research kit, using a monoclonal antibody detecting only the uncomplexed form of PAPP-A, did show a significantly higher concentration of PAPP-A in AMI when compared with controls.

In healthy controls, PAPP-A is present in forms complexed with or without proMBP. Uncomplexed PAPP-A originates from advanced atherosclerotic lesions [22], and is most likely produced by vascular endothelial and smooth muscle cells [13]. However, current commercially available ELISA kits for the detection of PAPP-A are designed to detect Down's syndrome and therefore focus on the total amount of PAPP-A present in serum (both complexed and uncomplexed with the proform of eosinophil major basic protein (proMBP)). In order to accurately assess the association and predictive value of PAPP-A levels in patients with acute coronary syndromes, the levels of uncomplexed PAPP-A need to be determined. This might explain the different results that were obtained between studies with custom made assays and commercially available assays. Future studies should focus on the association of this uncomplexed PAPP-A variant and cardiovascular disease [23]. In the current study, we demonstrated a significantly higher concentration of PAPP-A in AMI when compared with the other study groups using the ultrasensitive kit, and no detectable levels of PAPP-A after 6 months. 
Therefore, this ultrasensitive PAPP-A kit might aid in the detection of atherosclerosis-specific PAPP-A.

After 6 months, leukocyte counts and MPO concentrations remained significantly increased in AMI when compared with controls. Stepwise discriminant analysis of our data showed that leukocyte counts and MPO were the most powerful biomarkers, with regard to the accurate distinction between patients who had acute coronary events from those who did not (Table 3). Slightly less accurate but still reasonably high, was the discrimination between AMI, UAP, and a third group that combined SAP and CON. This time PAPP-A US contributed significantly to the other two biomarkers. Moreover, within the acute patients only (i.e. UAP and AMI), PAPP-A US showed a significant discriminative power, in combination with leukocyte counts. By contrast, once all four original diagnostic groups were considered, the classification of patients according to these three biomarkers was suboptimal as only $62 \%$ of the cases were classified correctly (Table 3). Thus, healthy controls and patients with SAP share similar biomarkers profiles, indicating that their clear-cut separation would require additional diagnostic criteria. Slightly better is the differentiation of acute and non-acute events based on patients' biomarker concentrations. On the one hand, MPO and leucocyte counts were promising biomarkers to distinguish between the two groups, whereas in combination with the PAPP-A US, an additional separation between non-acute (i.e. SAP and CON combined), UAP, and AMI patients was attained.

A curious finding was that, although MPO together with leukocyte counts was a significant discriminant for the acute versus the non acute patients, MPO itself was not able to further differentiate within the acute patients (UAP and AMI), in which case only PAPP-A US and leukocyte counts emerged as sufficiently discriminative (Table 3 ). These findings suggest that of the biomarkers tested in this study, leukocyte counts, MPO, and PAPP-A US have the most potential as biomarkers of acute cardiovascular disease.

However, although some biomarkers have a strong correlation with cardiovascular disease, their clinical application remains questionable. Several of the biomarkers analyzed showed a significant range in measured concentrations. This means that although there might be a significant increase in concentrations of the biomarkers when compared with controls, there are still individuals that have low concentrations of the biomarker. Therefore, it cannot be used as a biomarker the predict the individual's risk of future 
cardiovascular events. In addition, it emphasizes the need for a 'multimarker' approach, in which the concentrations of a certain panel of biomarkers is measured and used to predict the individual's risk of near-future cardiovascular events.

The present study showed that MPO and leukocyte counts were independent biomarkers, as demonstrated by the correlation matrix value of 0.5 in the stepwise discriminant analysis. Also, the levels of MPO and leukocyte counts were still significantly increased after 6 months follow-up, making them promising biomarkers for assessing future cardiovascular risk. Future studies should address the possibility of combining the most promising biomarkers available to investigate whether this approach leads to even higher relative risk estimates for future cardiovascular events.

In conclusion, leukocyte counts and levels of fibrinogen, CRP, myeloperoxidase, and pregnancy associated plasma protein-A were increased in patients with acute coronary syndromes. When combined, leukocyte counts, MPO, and PAPP-A US classified study group designation for acute coronary events correctly in $82 \%$ of the cases. 


\section{REFERENCES}

1. Hansson GK. Inflammation, atherosclerosis, and coronary artery disease. N Engl J Med 2005;352:1685-1695.

2. Lusis AJ. Atherosclerosis. Nature 2000;407:233-241.

3. Koenig W, Khuseyinova N. Biomarkers of atherosclerotic plaque instability and rupture. Arterioscler Thromb V asc Biol 2007;27:15-26.

4. Ridker PM. High-sensitivity C-reactive protein: potential adjunct for global risk assessment in the primary prevention of cardiovascular disease. Circulation 2001;103:18131818.

5. Fibrinogen Studies Collaboration. Plasma fibrinogen level and the risk of major cardiovascular diseases and nonvascular mortality: an individual participant meta-analysis. JAMA 2005;294:1799-1809.

6. Danesh J, Collins R, Appleby R, Peto R. Association of fibrinogen, C-reactive protein, albumin, or leukocyte count with coronary heart disease: meta-analyses of prospective studies. JAMA 1998;279:1477-1482.

7. Yarnell JW, Patterson CC, Sweetnam PM, Lowe GD. Haemostatic/inflammatory markers predict 10-year risk of IHD at least as well as lipids: the Caerphilly collaborative studies. Eur Heart J 2004;25:1049-1056.

8. Danesh J, Wheeler JG, Hirschfield GM, Eda S, Eiriksdottir G, Rumley A, Lowe GD, Pepys MB, Gudnason V. C-reactive protein and other circulating markers of inflammation in the prediction of coronary heart disease. N Engl J Med 2004;350:1387-1397.

9. Smith GD, Harbord R, Milton J, Ebrahim S, Sterne JA. Does elevated plasma fibrinogen increase the risk of coronary heart disease? Evidence from a meta-analysis of genetic association studies. Arterioscler Thromb V asc Biol 2005;25:2228-2233.

10. Baldus S, Heeschen C, Meinertz T, Zeiher AM, Eiserich JP, Münzel T, Simoons ML, Hamm CW, CAPTURE investigators. Myeloperoxidase serum levels predict risk in patients with acute coronary syndromes. Circulation 2003; 108:1440-1445.

11. Haim M, Boyko V, Goldbourt U, Battler A, Behar S. Predictive value of elevated white blood cell count in patients with preexisting coronary heart disease: the Bezafibrate Infarction Prevention Study. Arch Intern Med 2004; 164:433-439.

12. Barron HV, Harr SD, Radford MJ, Wang Y, Krumholz HM. The association between white blood cell count and acute myocardial infarction mortality in patients $>$ or $=65$ years of age: findings from the cooperative cardiovascular project. $J$ Am Coll Cardiol 2001;38:1654-1661.

13. Bayes-Genis A, Conover CA, Overgaard MT, Bailey KR, Christiansen M, Holmes DR Jr, Virmani R, Oxvig C, Schwartz RS. Pregnancy-associated plasma protein A as a marker of acute coronary syndromes. N Engl J Med 2001;345:1022-1029.

14. Zhang R, Brennan ML, Fu X, Aviles RJ, Pearce GL, Penn MS, Topol EJ, Sprecher DL, Hazen SL. Association between myeloperoxidase levels and risk of coronary artery disease. JAMA 2001;286:2136-2142.

15. Brennan ML, Penn MS, Van Lente F, Nambi V, Shishehbor MH, Aviles RJ, Goormastic M, Pepoy ML, McErlean ES, Topol EJ, Nissen SE, Hazen SL. Prognostic value of myeloperoxidase in patients with chest pain. N Engl J Med 2003;349:1595-1604.

16. Mocatta TJ, Pilbrow AP, Cameron VA, Senthilmohan R, Frampton CM, Richards AM, Winterbourn CC. Plasma concentrations of myeloperoxidase predict mortality after myocardial infarction. J Am Coll Cardiol 2007;49:1993-2000. 
17. Cavusoglu E, Ruwende C, Eng C, Chopra V, Yanamadala S, Clark LT, Pinksy DJ, Marmur JD. Usefulness of baseline plasma myeloperoxidase levels as an independent predictor of myocardial infarction at two years in patients presenting with acute coronary syndrome. Am J Cardiol 2007;99:1364-1368.

18. Ruef J, März W, Winkelmann BR. Markers for endothelial dysfunction, but not markers for oxidative stress correlate with classical risk factors and the severity of coronary artery disease. (A subgroup analysis from the Ludwigshafen Risk and Cardiovascular Health Study). Scand Cardiovasc J 2006;40:274-279.

19. Lund J, Qin QP, Ilva T, Pettersson K, Voipio-Pulkki LM, Porela P, Pulkki K. Circulating pregnancy-associated plasma protein a predicts outcome in patients with acute coronary syndrome but no troponin I elevation. Circulation 2003;108:1924-1926.

20. Heeschen C, Dimmeler S, Hamm CW, Fichtlscherer S, Simoons ML, Zeiher AM, CAPTURE Study Investigators. Pregnancy-associated plasma protein-A levels in patients with acute coronary syndromes: comparison with markers of systemic inflammation, platelet activation, and myocardial necrosis. J Am Coll Cardiol 2005;45:229-237.

21. Dominguez-Rodriguez A, Abreu-Gonzalez P, Garcia-Gonzalez M, Ferrer J, Vargas M. Circulating pregnancy-associated plasma protein $\mathrm{A}$ is not an early marker of acute myocardial infarction. Clin Biochem 2005;38:180-182.

22. Wittfooth S, Qin QP, Lund J, Tierala I, Pulkki K, Takalo H, Pettersson K. Immunofluorometric point-of-care assays for the detection of acute coronary syndromerelated noncomplexed pregnancy-associated plasma protein A. Clin Chem 2006;52:17941801.

23. Qin QP, Wittfooth S, Pettersson K. Measurement and clinical significance of circulating PAPP-A in ACS patients. Clin Chim Acta 2007;380:59-67.

\section{ACKNOWLEDGEMENTS}

We wish to acknowledge cardiologists and nurses of the Cardiology departments of the Maastricht University Hospital and the Maasland hospital in Sittard for their support in including patients.

S.H. and M.D. participate in the European Vascular Genomics Network (http://www.evgn.org), a Network of Excellence supported by the European Community's Sixth Framework Program for Research Priority 1 (Life Sciences, Genomics, and Biotechnology for Health; contract LSHM-CT2003-503254). This work was supported by the Dutch Heart Foundation, grant number 2002.B033, and by the 'Besluit Subsidies Investeringen Kennisinfrastructuur' (BSIK) program entitled 'Molecular imaging of ischemic heart disease (project number BSIK 03033). 



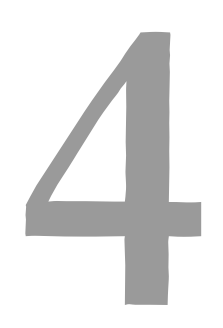

Contrast-enhanced magnetic resonance vessel wall imaging in a rabbit model of atherosclerosis: comparison of gadofosveset and Gd-DTPA

M.B.I. Lobbes, R.J.J.H.M. Miserus, S. Heeneman, V. Lima Passos, P.H.A. Mutsaers, N. Debernardi, B. Misselwitz, M. Post, M.J.A.P. Daemen, J.M.A. van Engelshoven, T. Leiner, M.E. Kooi 


\section{ABSTRACT}

Purpose: (Contrast-enhanced) magnetic resonance imaging has proven to be promising to image atherosclerotic plaques. Our aim was to investigate the potential of gadofosveset for plaque imaging in an atherosclerotic rabbit model.

Materials and methods: All experiments were approved by the animal ethics committee. Thirty-one New Zealand White rabbits were included in four study groups: atherosclerotic animals receiving gadofosveset $(n=10)$ or $\mathrm{Gd}-$ DTPA ( $\mathrm{n}=7$ ), and control animals receiving gadofosveset or Gd-DTPA (both $\mathrm{n}=7$ ). Aortic atherosclerosis was induced through endothelial denudation combined with a cholesterol-enriched diet. Control rabbits were sham operated and received regular diet. After eight weeks, pre- and post-contrast T1 weighted MR images of the aortic vessel wall were acquired. Relative signal enhancement was determined with dedicated software. Statistical analysis was performed using a generalized linear mixed model. CD31 and albumin immunohistochemical staining was used to assess microvessel density and albumin content of the vascular wall. Group differences were analyzed using a $\chi^{2}$-test. Gadofosveset spatial distribution and content within the vessel wall was determined with PIXE analysis.

Results: Post-contrast signal enhancement was significantly larger for atherosclerotic than for control animals using gadofosveset $(p=0.022)$. GdDTPA could not discriminate between normal and atherosclerotic vessel wall $(p=0.428)$. PIXE analysis showed higher amounts of Gd-DTPA in both atherosclerotic and normal rabbit aortas compared to gadofosveset. Immunohistochemical staining showed presence of albumin and increased microvessel density in the vascular wall of atherosclerotic rabbits.

Conclusion: Current results suggest that gadofosveset can be used to differentiate between atherosclerotic and normal rabbit vessel wall. 


\section{INTRODUCTION}

Atherosclerotic plaques develop over decades, leading to advanced lesions characterized by a lipid core separated from the lumen by a fibrous cap [1]. Clinical events (e.g. myocardial infarction) are caused by rupture or erosion of the fibrous cap accompanied by thromboembolization and occlusion of a diseased or distal vessel $[2,3]$. The risk of a clinical event is determined by plaque composition and degree of stenosis [4, 5]. Histologic studies demonstrated that macrophage-rich plaques with a thin fibrous cap, large lipid core, and abundance of (leaky) microvessels tend to be more vulnerable [4]. Intraplaque albumin content is positively associated with plaque size and micro-vessel density, which could be related to leakage of albumin into the lesion through leaky microvessels [6].

DSA and US are important diagnostic modalities to determine degree of stenosis in atherosclerosis, but DSA is unable to visualize plaque composition. In addition, identification of these components with US remains highly operator-dependent and has low reproducibility, despite recent improvements [7, 8]. MRI can non-invasively assess lumen diameter and plaque components [9-11]. However, limited spatial resolution and relatively low SNR of MRI makes it difficult to detect plaque components in small arteries, e.g. coronary arteries. MR contrast agents improve SNR and CNR and therefore might improve plaque imaging. Several gadolinium chelates, mostly Gd-DTPA, have been used for plaque imaging. After Gd-DTPA administration, increased signal enhancement occurs for fibrous tissue, improving contrast between fibrous tissue and the lipid core. Kinetic modeling of GdDTPA enhanced dynamic MRI can be used to quantify neovascularisation density [12-16].

Gadofosveset (MS-325, Vasovist ${ }^{\circledR}$, Bayer Schering Pharma AG, Germany) consists of a diphenylcyclohexyl group attached to a gadolinium chelate by a phosphodiester linkage. Since a large fraction $(>75 \%)$ of circulating gadofosveset is bound to serum albumin, it has a longer elimination half-life than Gd-DTPA. Bound gadofosveset gives a strong signal enhancement due to its high relaxivity $[17,18]$. In an experimental MRA study, Lin et al. observed signal enhancement in an occluded atherosclerotic pig carotid vessel wall after gadofosveset administration [19]. In the present study we aimed to investigate the potential of gadofosveset for plaque imaging in an atherosclerotic rabbit model. 


\section{MATERIALS AND METHODS}

\section{Animal experimental protocols}

Gadofosveset was donated by Bayer Schering Pharma AG, Germany. B.M. is an employee of Bayer Schering Pharma AG and provided us with critical comments on the manuscript. B.M. had no influence on animal experiments or data analysis.

Thirty-one male New Zealand White rabbits (12 weeks old, minimum weight $2.0 \mathrm{~kg}$ ) were randomized into four study groups. Animals were randomly assigned to either a 1.0\% cholesterol enriched diet (atherosclerotic animals, $n=17$ ) or normal rabbit chow (controls, $n=14$ ) for two weeks. Cholesterol-fed rabbits underwent an intervention consisting of a balloon denudation of the abdominal aorta, while control rabbits underwent sham surgery. During the balloon denudation or sham surgery, rabbits were anesthetized with ketamine $(0.5 \mathrm{ml} / \mathrm{kg})$ and xylazine $(0.25 \mathrm{ml} / \mathrm{kg})$. When sedated, animals were intubated with an endotracheal tube and actively ventilated using oxygen with $2 \%$ isoflurane inhalation gas. The left carotid artery was exposed and a $4 \mathrm{~F}$ catheter introducer sheath was inserted (Avanti Plus, Cordis Europe, Belgium) until the aortic arch was reached. Next, a small guidewire (Radiofocus Guidewire M, Terumo Europe, Belgium) was inserted through the sheath until it was situated in the descending aorta. Then, the guidewire was replaced with a small 3F Fogarty embolectomy catheter (Edwards Lifesciences, USA), which was positioned $1 \mathrm{~cm}$ proximally to the aortic bifurcation. From this starting point, the catheter was inflated and withdrawn $5 \mathrm{~cm}$ for three times. After removal of the catheter, the carotid artery was ligated and the wound was sutured. The sham surgery of control rabbits (receiving normal chow) consisted of a ligation of the left carotid artery. No catheterization material was introduced.

After surgery, animals received their assigned diets for eight weeks and underwent both pre- and post-contrast MR examination of the abdominal aorta. Between pre- and post-contrast MRI experiments, ten atherosclerotic and seven controls rabbits received an intravenous injection of gadofosveset $(0.05 \mathrm{mmol} / \mathrm{kg})$, whereas seven atherosclerotic and seven controls rabbits received Gd-DTPA (Magnevist ${ }^{\circledR}, 0.5 \mathrm{mmol} / \mathrm{kg}$ ) (both Bayer Schering Pharma AG, Germany).

Technical outliers were defined as rabbits in which development of atherosclerotic plaque was significantly less compared to other animals, even 
though identical experimental protocols were used. These animals $(\mathrm{n}=2)$ were excluded from analysis. Animals were sacrificed after the final experiment with an overdose of sodium pentobarbital. All experiments were approved by the local animal ethics committee.

\section{MR imaging}

Acquisitions were performed using a 1.5T MRI scanner (Philips Healthcare, the Netherlands) and a five-element synergy receiver cardiac coil. A twodimensional FFE sequence was used to identify the aortic bifurcation. A three-dimensional, DIR (black blood) TSE pulse sequence was performed. In this sequence, a CLEAR technique was used to compensate for signal inhomogeneities. The central imaging slice was placed 3.5 centimetres above the aortic bifurcation.

The doses of both contrast agents were chosen to compensate relaxivity differences between the contrast agents (free gadofosveset $6.0 \mathrm{mM}^{-1} \mathrm{~s}^{-1}$, bound gadofosveset $33.0 \mathrm{mM}^{-1} \mathrm{~s}^{-1}$, Gd-DTPA $4.5 \mathrm{mM}^{-1} \mathrm{~s}^{-1}$ ). We calculated that with these doses, $T_{1}$ values of blood immediately after contrast administration were comparable for both contrast agents. Optimal post-contrast imaging time was determined based on pilot experimental data (see below). At four times the elimination half-life of the administered contrast agent, post-contrast imaging was performed. For gadofosveset and Gd-DTPA these periods were 11 hours and 90 minutes, respectively.

For the two-dimensional FFE sequence, the following scan parameters were used: TR $17 \mathrm{~ms}$, TE $3.8 \mathrm{~ms}$, flip angle $60^{\circ}$. The three-dimensional, DIR (black blood) TSE pulse sequence was performed with the following scan parameters: echo train length 5, echo train spacing $14 \mathrm{~ms}$, TR $570 \mathrm{~ms}$, TE $14 \mathrm{~ms}$, TI $252 \mathrm{~ms}$, NSA 2, 9 slices of $3.0 \mathrm{~mm}$ thickness, FOV 90x90 mm, matrix size $304 \times 304$, resulting in an in-plane resolution of $0.3 \times 0.3 \mathrm{~mm}$. TI was not adjusted during post-contrast imaging, since after four times the elimination half-life, T1 values of blood restored to their original values. A regional saturation slab was placed over the bowels to prevent bowel motion artefacts in the abdominal aorta.

In the MR image analysis, an operator-defined ROI was drawn encompassing the arterial vessel wall. This ROI was copied from pre-contrast to postcontrast images, adjusting only for minor displacements that occurred between the two scans. ROIs were placed independently by $2 \mathrm{MR}$ readers blinded for study group (M.L., R.M.). Mean SI of both pre- and post- 
contrast images were adjusted for scaling factors and gain. Mean precontrast SI of adjacent muscle tissue, again adjusted for scaling factors and gain, was also determined in an operator-defined ROI. Pre- and postcontrast relative vessel wall signal intensities were determined by dividing mean signal intensities (pre- or post-contrast) by the pre-contrast mean SI of adjacent muscle tissue. Signal enhancement was calculated by dividing the post-contrast SI by the pre-contrast SI.

To determine the optimal post-contrast imaging time, a pilot experiment was conducted, in which a single atherosclerotic rabbit was continuously imaged for 13 hours after administration of gadofosveset $(0.05 \mathrm{mmol} / \mathrm{kg})$. In this experiment, the mean signal enhancement of the vessel wall increased to 3.1 after 72 minutes, after which it decreased to 2.4 after 13 hours of imaging, when the rabbit was sacrificed. As a trade off between maximum signal enhancement of the vessel wall versus minimal signal enhancement of the lumen, we opted for a post-contrast imaging delay of 11 hours for the gadofosveset groups (equalling four times the elimination half-life). Post-contrast imaging of Gd-DTPA receiving animals would have to performed at a similar elimination half-life, taken into account the differences in elimination half-life of the contrast agents. Therefore, the post-contrast imaging delay for Gd-DTPA groups was 90 minutes, again four times the elimination half-life of the contrast agent.

\section{MR image analysis}

MR image analysis was performed with VesselMASS (Leiden University Medical Center, the Netherlands). In the images, operator defined ROI were drawn by two independent readers (M.L., R.M., both over three years of experience) [64]. One reader blinded for study groups and MRI results determined overall image quality (M.E.K., over nine years of experience). Images in which contours of the vessel wall could not be distinguished from lumen or surrounding tissue, were excluded from analysis.

\section{Histological analysis}

After euthanasia, the aorta was dissected from bifurcation to approximately $7 \mathrm{~cm}$ proximally. The aortas of seven atherosclerotic and seven control rabbits were formalin fixed for 24 hours, after which they were cut into $3 \mathrm{~mm}$ thick tissue slices (taking into account shrinkage that occurred during the fixation process) and embedded in paraffin. For PIXE analysis and immunohistochemical staining, tissue slices of the remaining nineteen rabbits 
were embedded in Tissue-Tek (Electron Microscopy Sciences, USA) and snap frozen in dry-ice cooled isopentane. Paraffin and cryosections were cut into 4 and $10 \mu \mathrm{m}$ thick slices, respectively. Section of poor quality, i.e. broken or incomplete sections, were excluded from analysis. All sections were Lawson and HE stained and microscopically analyzed. Digital photographs of Lawson stained sections were subsequently analyzed with ImageJ 1.37 (National Institute of Health, USA) to assess plaque size. All sections were immunohistologically stained for endothelial cells with CD31 (clone JC70A, Dako North America Inc., USA) and assessed for microvessel density. Presence and localization of albumin was assessed with immunohistochemical staining using a human serum albumin antibody (AB8940, Abcam Inc., USA,).

The amount of micro-vessels in completely intact samples was assessed and scored on a 5-point scale: none (0), few (1: 1-10 vessels per cross-section), minor (2: 11-20 vessels), average (3: 21-30 vessels) and abundant (4: >30 vessels). Albumin content was measured by dividing the total area that stained positive by total plaque area, and multiplying this by 100 . Thus, the albumin content was expressed as the percentage of total plaque area that stained positive. Analysis of the albumin staining was quantified using computerized morphometry (Leica QWin V3, Cambridge, United Kingdom).

In both immunohistochemical stainings, proper positive and negative controls were used and sections were stained in one batch. For CD31, positive controls consisted of human carotid atherosclerotic plaques, whereas for albumin, positive controls consisted of human breast carcinoma. In both stainings, negative controls consisted of omission of the first antibody.

\section{Proton Induced X-ray Emission analysis}

PIXE analysis was used to investigate contrast agent distribution within the vascular wall [21]. Two cryosections of two individual rabbits of each group were cut into $10 \mu \mathrm{m}$ thick slices, placed on pioloform film and compared with adjacent HE-stained samples. The detection limit of gadolinium and elemental concentrations expressed in $\mu \mathrm{g} / \mathrm{g}$ were calculated. Subsequently, distribution maps were made.

PIXE measurements were performed with a $3 \mathrm{MeV}$ proton beam from a 3.5 MV Singletron ${ }^{\mathrm{TM}}$. To encompass the entire width of the vessel wall, a scanning pattern of $128 \times 128$ points and a proton beam size of approximately $8 \mathrm{x} 8 \mu \mathrm{m}^{2}$ was used. Since elemental concentrations are measured over the 
entire grid, the grid was placed as accurately as possible to encompass the entire vessel wall.

\section{Statistical analysis}

ICC between the two MR readers were calculated to determine interobserver agreement. Pre- and post-contrast rSI measurements were analysed with a generalised linear mixed model using SPSS 15.0 (SPSS Inc., Chicago, USA) [66]. In the generalized linear mixed model, individual rabbits were treated as random factor and contrast agent and intervention as fixed effects. For the analysis of post-contrast rSI, pre-contrast rSI was added to the model as a covariate in order to correct for differences in baseline measurements among groups. Model parameters were considered significant if $\mathrm{p}-$ values of their corresponding Wald tests were $\mathrm{p}<0.05$. For those parameters whose $\mathrm{p}<0.1$, an additional LR test was carried out to determine their final significance. The final model parameter estimates were computed via the REML method. The LR tests were carried out with maximum likelihood estimates.

Mean scores for microvessel density were assessed for both atherosclerotic and normal aortas by dividing total micro-vessel density score by number of slices assessed. A $\chi^{2}$-test was performed to evaluate differences in microvessel densities and percentages of vessel wall areas positively stained for albumin. P-values of $<0.05$ were considered to be statistically significant.

\section{RESULTS}

Although identical experimental protocols were applied, two atherosclerotic rabbits that received gadofosveset showed minimal plaque development (mean plaque size $0.8 \pm 0.7 \mathrm{~mm}^{2}$ versus $3.3 \pm 1.7 \mathrm{~mm}^{2}$ in other atherosclerotic rabbits). We considered these rabbits as technical outliers and excluded them from further analysis.

\section{MRI results}

A total of 558 images were acquired. Due to poor vessel wall delineation in MR images, 52 images ( $9 \%$ ) out of 13 different animals were excluded. Typical examples of MR images per study group are presented in Figure 1. 
Interobserver agreement as expressed by the ICC for pre- and post-contrast rSI of the vessel wall was 0.951 and 0.986 , respectively (all $\mathrm{p}<0.001$ ).

Figure 2a shows box plots of rSI baseline values for each rabbit, clustered by group. Atherosclerotic groups showed a higher pre-contrast rSI than controls. To check whether pre-contrast rSI values were significantly different among rabbits, baseline measurements were analysed via a mixed model with random intercept and contrast and illness as fixed effects. A statistically significant higher pre-contrast rSI was found for atherosclerotic rabbits: mean pre-contrast rSI difference between atherosclerotic and normal animals was $0.264(\mathrm{p}<0.001)$. Mean pre-contrast rSI difference between contrast groups (gadofosveset or Gd-DTPA) was $0.042(\mathrm{p}=0.196$, note that no contrast has been added at this stage).

Box plots for observed post-contrast SE showed that with exception of five animals, mean post-contrast SE increased with at least a factor of 1.5 after contrast administration (Figure 2b). Five animals that showed limited increase in post-contrast SE were control animals that received gadofosveset.

A mixed model with random intercept and random slope on the precontrast rSI was fit to the post-contrast measurements. Final fixed effects parameters are given in Table 1.

Table 1. Final model parameters for fixed effects. P-value for Wald test is presented. For illness and contrast, atherosclerosis and Gd-DTPA served as references, respectively.

\begin{tabular}{llll}
\hline Covariate & Regression coefficient & Standard error & Wald p-value \\
\hline Intercept & 1.397 & 0.243 & $<0.001$ \\
Illness & -0.205 & 0.255 & 0.428 \\
Contrast & -1.326 & 0.244 & $<0.001$ \\
Pre-contrast rSI & 1.477 & 0.178 & $<0.001$ \\
Interaction illness/contrast & 0.801 & 0.352 & 0.032 \\
\hline
\end{tabular}

In Figure 3 observed post-contrast rSI values for each individual rabbit were plotted against their corresponding pre-contrast rSI measurements. Additionally, the regression lines, as predicted by the fixed effects only (yet estimated under the mixed model), were displayed. 

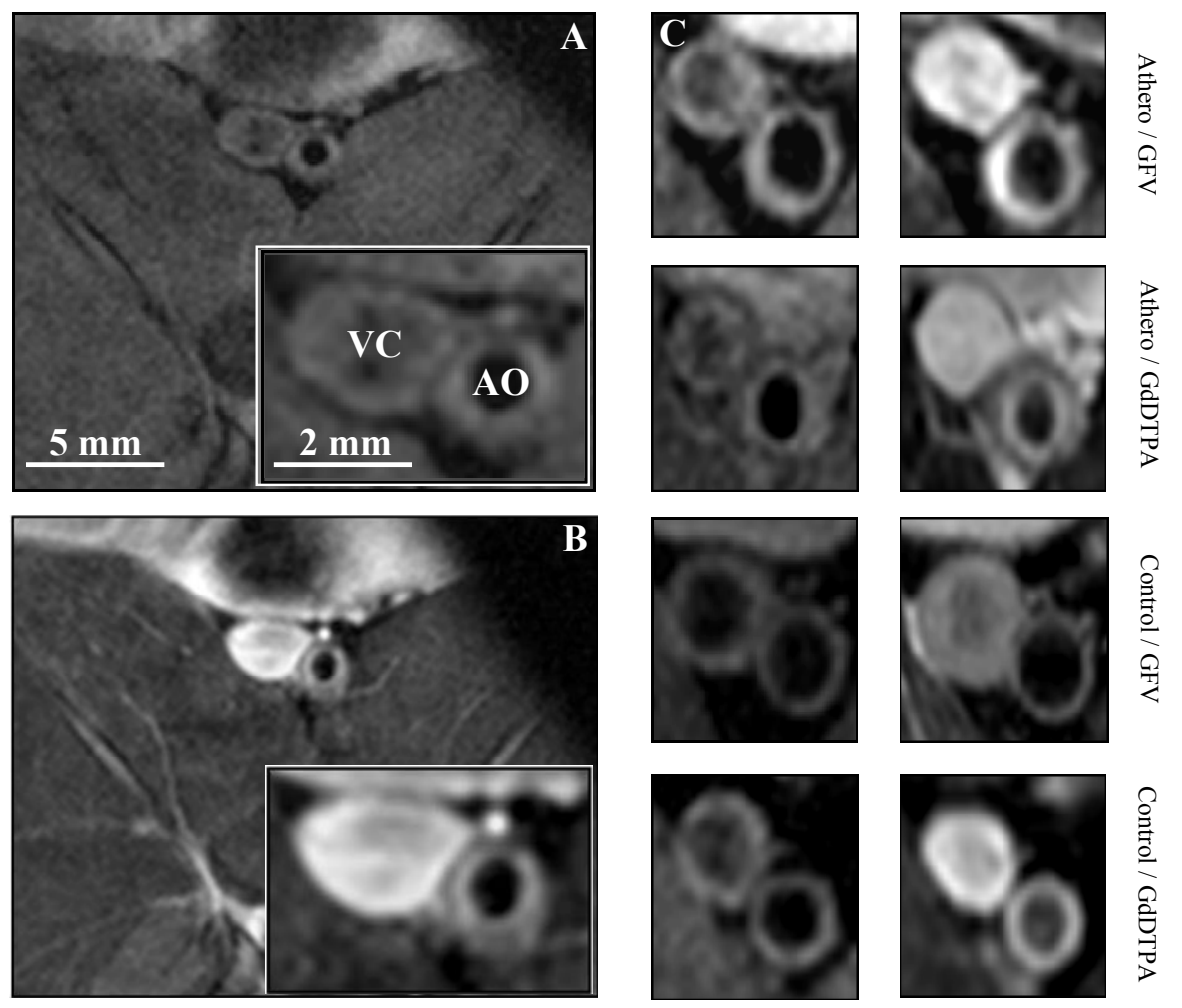

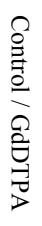

Figure 1. Overview of typical pre- (A) and post-contrast (B) MR images. Detailed MR images of all study groups $(C)$ show signal enhancement after contrast administration in all groups except controls receiving gadofosveset. Abbrevations: aorta (AO), inferior vena cava (VC), gadofosveset (GFV).

The significant interaction between lesion development and contrast suggests that there is a differential effect of the presence of atherosclerosis on post-contrast rSI, depending on the contrast applied. In order to illustrate the interaction effect, pair-wise comparisons based on estimated group means (Table 2) were carried out by holding the pre-contrast rSI value constant at its mean value of 1.176 . A statistically significant difference could be shown between atherosclerotic and control rabbits receiving gadofosveset (mean post-contrast $\mathrm{rSI}$ difference of $0.595, \mathrm{p}=0.022$ ). There was no significance between atherosclerotic rabbits and controls receiving Gd-DTPA (mean post-contrast $\mathrm{rSI}$ difference $0.205, \mathrm{p}=0.428$ ). Moreover, a differential contrast effect could be observed, with higher post-contrast signal difference between gadofosveset and Gd-DTPA for controls (mean post-contrast 

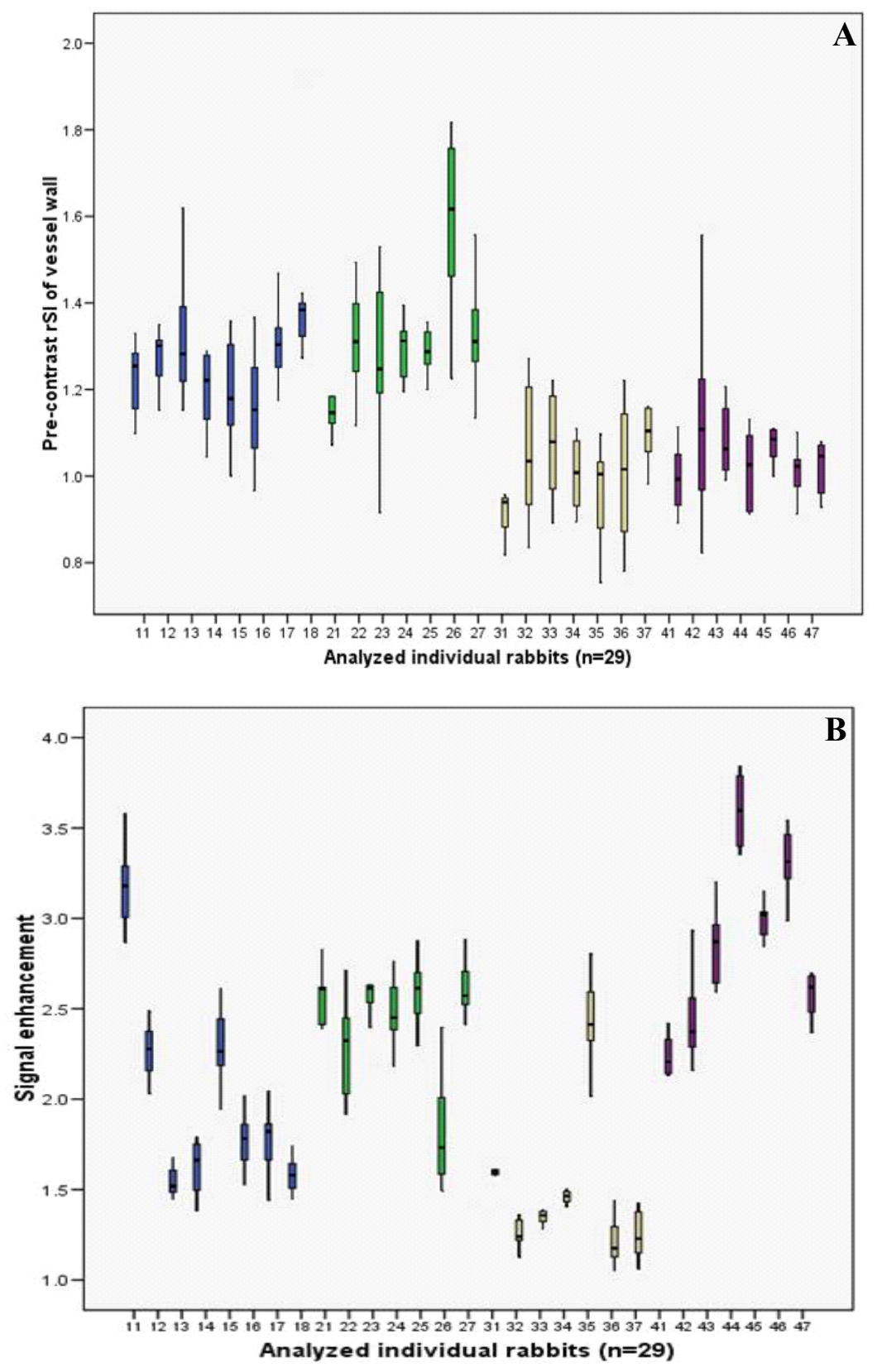

Figure 2. Box plots of pre-contrast rSI values (A) and signal enhancement (B) for each rabbit, clustered by groups: atherosclerosis/gadofosveset (blue), atherosclerosis/GdDTPA (green), controls/gadofosveset (beige), controls/GdDTPA (purple). 
rSI difference of $1.326, \mathrm{p}<0.001$ ) than for atherosclerotic rabbits (mean post-contrast rSI difference $0.526, \mathrm{p}=0.047$ ). These observed differences remained statistically significant even when the two technical outliers were included in the analysis (data not shown).

One control rabbit receiving gadofosveset showed increased signal enhancement after 11 hours. A similar experimental protocol was followed and no evident plaques, albumin content or increased microvessel density was observed in histological sections. Therefore, we have no explanation for this outlier. Despite this outlier, a significant difference in signal enhancement after gadofosveset administration was observed between atherosclerotic and control rabbits.

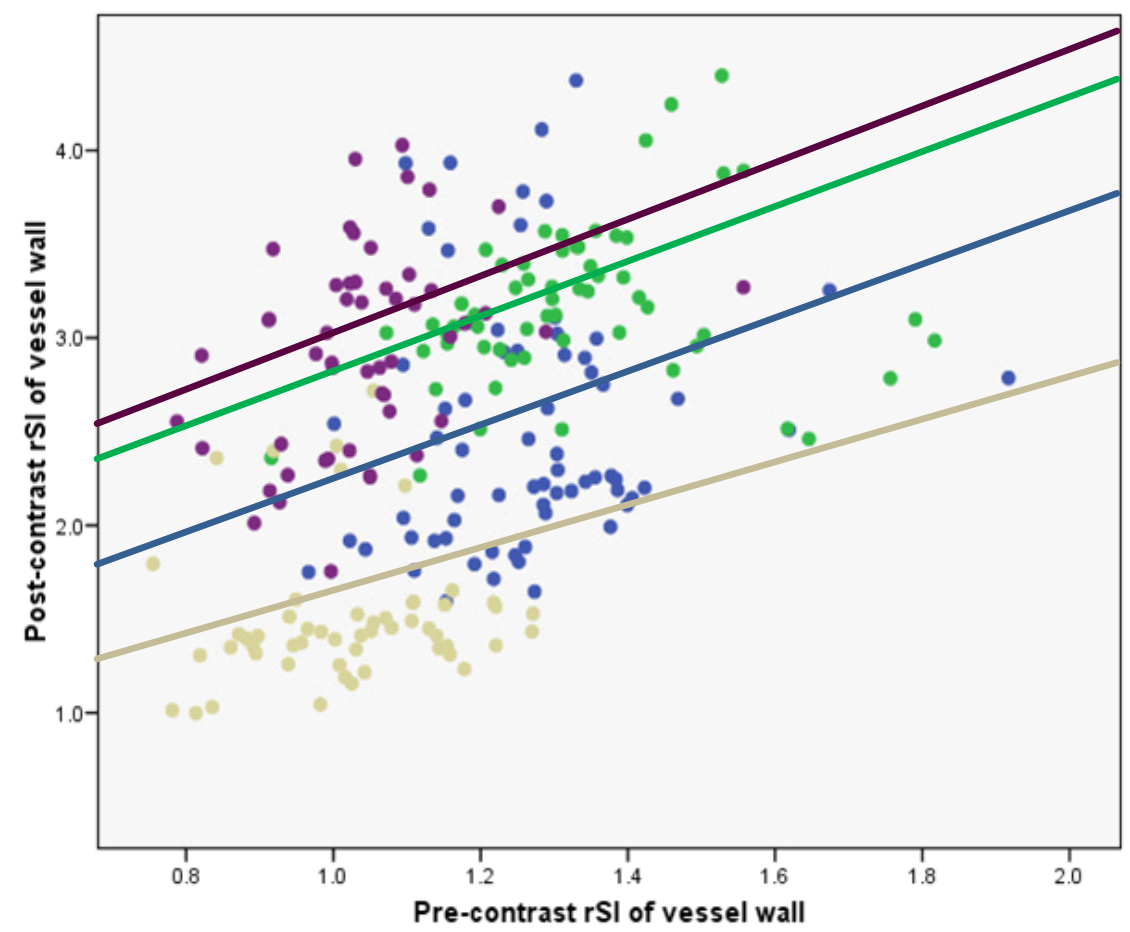

Figure 3. Observed post-contrast rSI as a function of pre-contrast rSI for individual rabbits per group. The regression lines are based on the predicted post-contrast rSI values for fixed effects only. Colours are indicative of study groups: atherosclerosis/gadofosveset (blue), atherosclerosis/GdDTPA (green), controls/gadofosveset (beige), and controls/GdDTPA (purple). 
In summary, post-contrast mean signal enhancement was significantly larger for atherosclerotic than for control animals using gadofosveset. For nondiseased control rabbits, Gd-DTPA yielded a larger post-contrast MR signal enhancement than gadofosveset. These results show that gadofosveset, in contrast to Gd-DTPA, is able to distinguish between atherosclerotic and normal vessel wall.

Table 2. Estimated means with corresponding standard errors upon which pairwise comparison is based at pre-contrast rSI of 1.176.

\begin{tabular}{lll}
\hline Study group & Estimated means & Standard error \\
\hline Atherosclerosis - gadofosveset & 2.402 & 0.173 \\
Atherosclerosis - Gd-DTPA & 2.928 & 0.188 \\
Controls - gadofosveset & 1.807 & 0.177 \\
Controls - Gd-DTPA & 3.133 & 0.178 \\
\hline
\end{tabular}

\section{Histology}

In atherosclerotic rabbits, macrophage-rich and collagen-poor lesions were formed (Figure $4 \mathrm{a}$, mean plaque size $3.3 \pm 1.7 \mathrm{~mm}^{2}$ ). All non-denudated control rabbits were free of atherosclerotic disease.

In the analysis of microvascular density and albumin content, $15 \%$ of the histological samples were not intact and excluded. An increase in microvascular density was observed, mainly in the adventitia and in five sections of two separate atherosclerotic animals also in the neointima (Figure 4c and 5c). In control animals, occasional adventitial microvessels were seen (Figure 4c). There was a significant difference in mean microvessel density scores ( \pm standard error) between atherosclerotic and normal vessels: $2.6 \pm 1.1$ and $0.7 \pm 0.4(\mathrm{p}<0.001)$, respectively.

The percentage of area ( \pm standard error) that stained red in sections that were immunohistochemically stained for albumin was significantly higher in atherosclerotic rabbits compared with controls: $43.9 \pm 4.2 \%$ versus $9.4 \pm 2.3 \%$, respectively ( $<<0.001$, Figure 5). 
ATHEROSCLEROTIC GROUP
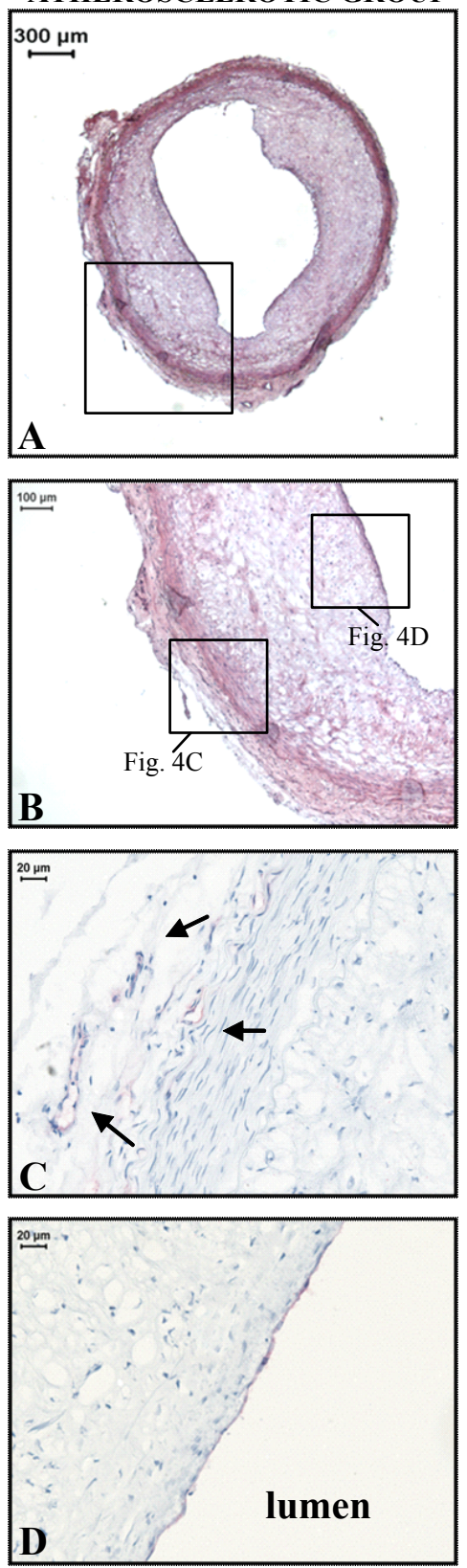

CONTROL GROUP
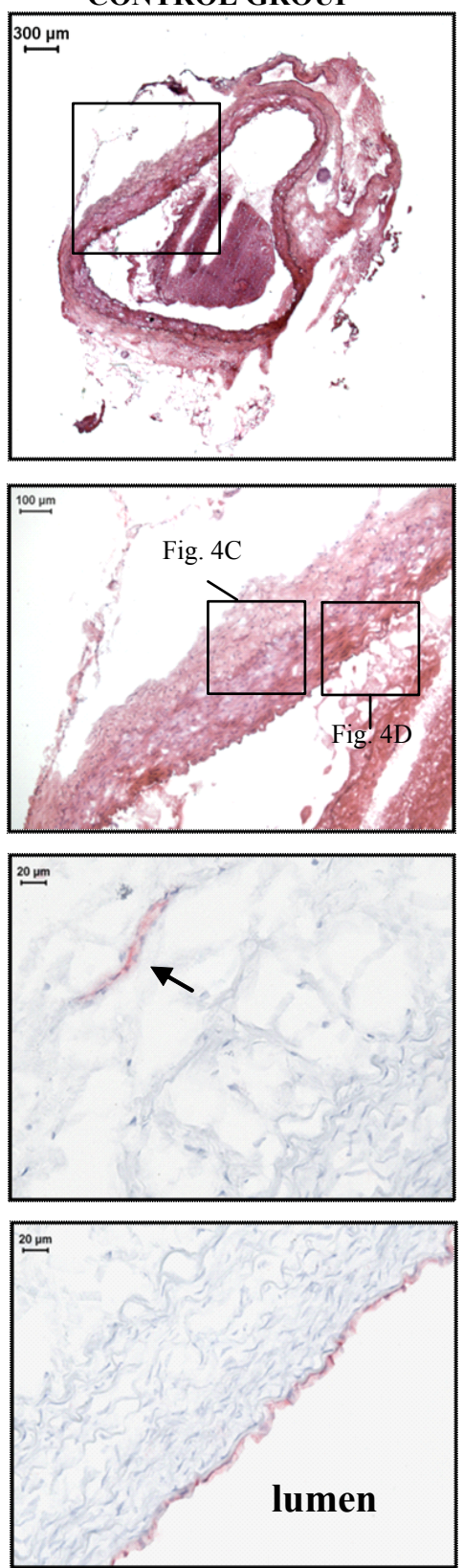

Figure 4. Histological examples of the aortic vessel wall of atherosclerotic and control rabbits. (A) HE stained anatomical overview. (B) HE stained detail of vessel wall. (C) CD31 immunoreactivity of adventitial microvessels visualized with Vector Red. (D) Positive CD31 red staining of endothelium. 

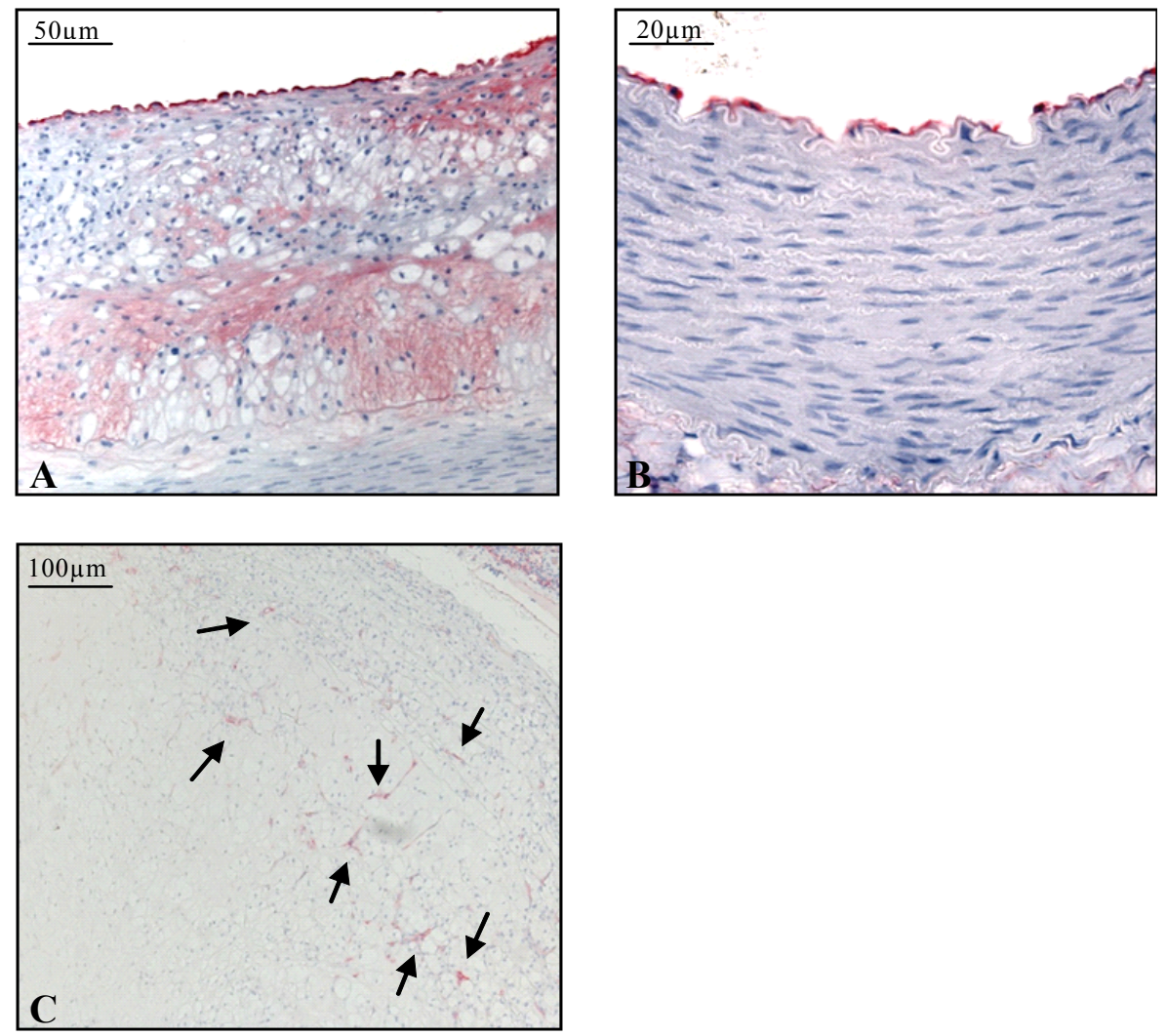

Figure 5. Immunohistochemical staining for albumin in vessel walls. Albumin is present in the atherosclerotic vessel wall lesions (A) and not in control animals (B). CD31 immunoreactive neointimal microvessels (arrows) might serve as port of entry (C). Intraluminal staining is caused by albumin-containing blood remnants and demonstrates positive staining for albumin in both cases.

\section{PIXE results}

PIXE analysis showed diffuse presence of gadolinium across the entire vessel wall (Figure 6). In atherosclerotic rabbits that received gadofosveset, the mean amount of gadolinium was $79 \mu \mathrm{g} / \mathrm{gram}$ dry tissue (range 16-248 $\mu \mathrm{g} / \mathrm{g}$ ). In control rabbits that received gadofosveset, the mean gadolinium content was $64 \mu \mathrm{g} / \mathrm{g}$ (range $6-602 \mu \mathrm{g} / \mathrm{g}$ ). In contrast, mean gadolinium content in groups that received Gd-DTPA was higher: in atherosclerotic rabbits $408 \mu \mathrm{g} / \mathrm{g}$ (range $97-2967 \mu \mathrm{g} / \mathrm{g}$ ) and in control rabbits $776 \mu \mathrm{g} / \mathrm{g}$ (range $91-1875 \mu \mathrm{g} / \mathrm{g}$ ). When corrected for clearance and dose, the amount of gadolinium in the study groups was similar. 

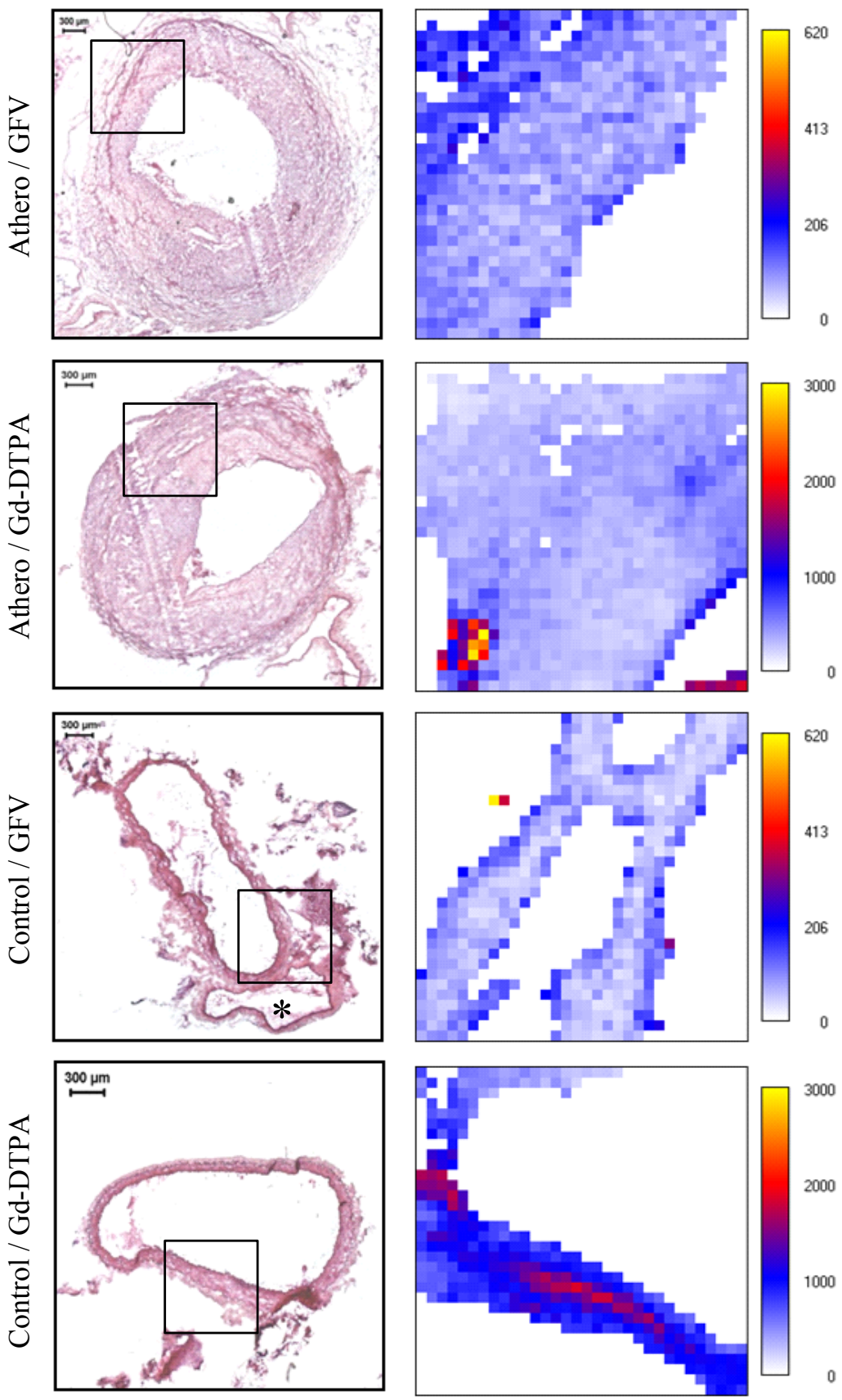

Figure 6. PIXE analysis. HE stained sections served as orientation point (square) for the analysis of the distribution of gadolinium. Colours are indicative of the amount of gadolinium present. Scaling of bars is in micrograms per gram dry tissue. $\left(^{*}\right)$ Inferior vena cava. 


\section{DISCUSSION}

Our study demonstrated that gadofosveset showed specific MR signal enhancement in the atherosclerotic vessel wall, whereas Gd-DTPA could not discriminate between normal and atherosclerotic rabbit vessel wall. Therefore, use of gadofosveset for differentiation between atherosclerotic and normal vessel walls is promising.

In an experimental MRA study, Lin et al. described an increased signal intensity in an occluded atherosclerotic pig carotid after gadofosveset administration. Increased neovascularisation was suggested as a possible explanation [19]. In our study, we confirmed and quantified the signal enhancement after gadofosveset administration in a large group of animals (rabbit atherosclerotic vessel walls) and demonstrated limited enhancement in nondiseased vessel walls. The presence of albumin might play an important role in this phenomenon.

After administration, gadofosveset $(>75 \%)$ is bound to serum albumin, resulting in an increased elimination half-life and prolonged time window for uptake in atherosclerotic plaques. Binding of gadofosveset to albumin increases $\mathrm{r}_{1}$ relaxivity from 6.0 (free) to $33.0 \mathrm{mM}^{-1} \mathrm{~s}^{-1}$ (bound), resulting in significant MR signal enhancement even at low doses [17, 23]. Albumin is the most abundant protein present in human circulation, accounting for approximately $60 \%$ of the measured serum proteins [24]. Normal arterial vessel walls do not contain albumin, since albumin leaves the circulation only through the fenestrated endothelium of capillaries. Albumin can enter the atherosclerotic vessel wall as demonstrated by Zhang et al., who showed a positive correlation between plaque size, micro-vessel density, and plaque albumin content [6]. The albumin content present in atherosclerotic lesions was significantly higher compared to plasma albumin concentration [25]. Gadofosveset binds to albumin and might enter the plaque in a similar way as native albumin. Alternatively, the relatively small free gadofosveset might be able to diffuse through the microvasculature or cross the endothelium into the plaque. Here it encounters albumin, resulting in binding of gadofosveset to albumin and increased relaxivity, creating a measurable amount of MR signal enhancement, even when low concentrations of free gadofosveset have entered the atherosclerotic vessel wall. Since no endothelial dysfunction is present in normal arterial vessel walls, bound gadofosveset is expected to be unable to enter the arterial vessel wall. Free gadofosveset could be able to diffuse through the endothelium into the normal vessel wall 
but does not encounter albumin to increase its relaxivity. In both cases, no apparent post-contrast signal enhancement will be detected in normal vessel wall, especially when low doses of gadofosveset are used as in the present study.

Post-contrast signal intensity was increased in the vessel wall of atherosclerotic and normal animals receiving Gd-DTPA. These results are in agreement with results of a study published by Barkhausen et al., who could not distinguish between Watanabe rabbit wall segments with or without plaque burden directly after administration of Gd-DTPA [26]. In their study, signal intensities restored 60 minutes after contrast administration, whereas signal intensities were still increased in our New Zealand White rabbits after 90 minutes, even though similar doses were applied. Differences in pharmacokinetic behaviour of the contrast agent in Watanabe and New Zealand White rabbits might explain this discrepancy.

Studies by Barkhausen et al. and Zheng et al. demonstrated the specific enhancement of atherosclerotic plaques in Watanabe rabbits after administering gadofluorine [27]. No signal enhancement was observed in the vessel wall of control rabbits. Gadofluorine has a prolonged elimination half-life compared to Gd-DTPA. In combination with the increased permeability of atherosclerotic vessel walls this could explain the specific signal enhancement of plaques observed in these studies. Although gadofosveset and gadofluorine could thus be used for similar applications, gadofosveset is currently commercially available and allows for more rapid human applications and studies. In addition, novel contrast agents are being developed and evaluated for their use in MR plaque imaging. For example, Brushett et al. demonstrated the potential use of motexafin gadolinium in MR plaque imaging of deep-seated arteries [28].

PIXE analysis showed comparable concentrations of gadolinium in both atherosclerotic and normal vessel walls from rabbits that received gadofosveset. The albumin content was significantly higher in atherosclerotic rabbits compared to controls. This supports our hypothesis that small amounts of gadofosveset are taken up in normal as well as atherosclerotic vessel walls, but that binding to albumin and the subsequent increase in relaxivity causes the higher signal enhancement observed in atherosclerotic animals. In atherosclerotic rabbits, increased numbers of microvessels were present in the adventitia and occasionally in the neointima. These microvessels could function as a port of entry for albumin and gadofosveset. The small 
amount of gadofosveset in normal vessel wall increased the signal only slightly. The increased post-contrast signal intensities of all rabbits that received Gd-DTPA was confirmed by the large amount of gadolinium present in both atherosclerotic and normal vessel wall.

\section{Study limitations}

Measurement of the vessel wall signal intensities can be affected by partial volume effects due to the limited in-plane resolution in relation to the small vessel wall thickness. However, the error that can occur by this effect is comparable for all four study groups and therefore, we assume that it has no influence on final study outcomes. In addition, the longitudinal inhomogeneity of the plaque thickness is much smaller than in the axial plane. Therefore, we have chosen a higher resolution in the axial direction that in the longitudinal direction.

The violation of the linear model assumption regarding groups' homogeneity of variances could be regarded as a statistical limitation of the conducted analysis. Accordingly, p-values of the statistical tests should be interpreted cautiously. Log transformation of post- and pre-contrast rSI values, intended to make group's variances more uniform, was carried out and ensuing analysis (under the same mixed model) resulted in fully concordant results (data not shown). As such, they substantiated the statistical significance of the groups' comparisons and the conclusions drawn.

\section{Practical applications}

It has been shown by a large number of studies that MRI is a diagnostic modality to non-invasively image carotid plaque components, such as the lipid core, the fibrous cap, calcifications, and intraplaque haemorrhage [811]. MR plaque imaging of the small, deeper situated coronary arteries, remains extremely challenging due to the limited spatial resolution and low SNR of MRI. The use of gadofosveset in contrast-enhanced MR plaque imaging could enable specific visualization of atherosclerosis in these arteries. Future studies are warranted to determine whether gadofosveset is a valuable tool for human plaque visualization. 
Chapter 4

\section{CONCLUSION}

Gadofosveset showed specific MR signal enhancement in the atherosclerotic rabbit vessel wall, whereas Gd-DTPA could not discriminate between the normal and atherosclerotic vessel wall. Therefore, gadofosvesetenhanced MR plaque imaging might be used to differentiate between atherosclerotic and normal vessel walls. 


\section{REFERENCES}

1. Thom T, Haase N, Rosamond W, Howard VJ, Rumsfeld J, Manolio T, Zheng ZJ, Flegal K, O'Donnell C, Kittner S, Lloyd-Jones D, Goff DC Jr, Hong Y, Adams R, Friday G, Furie K, Gorelick P, Kissela B, Marler J, Meigs J, Roger V, Sidney S, Sorlie P, Steinberger J, Wasserthiel-Smoller S, Wilson M, Wolf P; American Heart Association Statistics Committee and Stroke Statistics Subcommittee. Heart disease and stroke statistics: 2006 update: a report from the American Heart Association Statistics Committee and Stroke Statistics Subcommittee. Circulation. 2006; 113: e85-151.

2. Lusis AJ. Atherosclerosis. Nature. 2000; 407: 233-41.

3. Hansson GK. Inflammation, atherosclerosis, and coronary artery disease. N Engl J Med. 2005; 352: 1685-95.

4. Virmani R, Kolodgie FD, Burke AP, Farb A, Schwartz SM. Lessons from sudden coronary death: a comprehensive morphological classification scheme for atherosclerotic lesions. Arterioscler Thromb Vasc Biol. 2000; 20: 1262-75.

5. Virmani R, Kolodgie FD, Burke AP, Finn AV, Gold HK, Tulenko TN, Wrenn SP, Narula J. Atherosclerotic plaque progression and vulnerability to rupture: angiogenesis as a source of intraplaque hemorrhage. Arterioscler Thromb Vasc Biol. 2005; 25: 2054 61.

6. Zhang Y, Cliff WJ, Schoefl GI, Higgins G. Immunohistochemical study of intimal microvessels in coronary atherosclerosis. Am J Pathol. 1993; 143: 164-72.

7. Kern R, Szabo K, Hennerici M, Meairs S. Characterization of carotid artery plaques using real-time compound B-mode ultrasound. Stroke. 2004; 35: 870-5.

8. Fayad ZA, Fuster V. Clinical imaging of the high-risk or vulnerable atherosclerotic plaque. Circ Res. 2001; 89: 305-16.

9. Fayad ZA, Fuster V. Characterization of atherosclerotic plaques by magnetic resonance imaging. Ann N Y Acad Sci. 2000; 902: 173-86.

10. Yuan C, Kerwin WS. MRI of atherosclerosis. J Magn Reson Imaging. 2004; 19: 710-9.

11. Leiner T, Gerretsen S, Botnar R, Lutgens E, Cappendijk V, Kooi E, van Engelshoven J. Magnetic resonance imaging of atherosclerosis. Eur Radiol. 2005; 15: 1087-99.

12. Wasserman BA, Casal SG, Astor BC, Aletras AH, Arai AE. Wash-in kinetics for gadolinium-enhanced magnetic resonance imaging of carotid atheroma. J Magn Reson Imaging. $2005 ; 21: 91-5$.

13. Aoki S, Aoki K, Ohsawa S, Nakajima H, Kumagai H, Araki T. Dynamic MR imaging of the carotid wall. J Magn Reson Imaging. 1999; 9: 420-7.

14. Yuan C, Kerwin WS, Ferguson MS, Polissar N, Zhang S, Cai J, Hatsukami TS. Contrast-enhanced high resolution MRI for atherosclerotic carotid artery tissue characterization. J Magn Reson Imaging. 2002; 15: 62-7.

15. Cai J, Hatsukami TS, Ferguson MS, Kerwin WS, Saam T, Chu B, Takaya N, Polissar NL, Yuan C. In vivo quantitative measurement of intact fibrous cap and lipid-rich necrotic core size in atherosclerotic carotid plaque: comparison of high-resolution, contrast-enhanced magnetic resonance imaging and histology. Circulation. 2005; 112: 3437 44.

16. Kerwin WS, O'Brien KD, Ferguson MS, Polissar N, Hatsukami TS, Yuan C. Inflammation in carotid atherosclerotic plaque: a dynamic contrast-enhanced MR imaging study. Radiology. 2006; 241: 459-68. 
17. Corot C, Violas X, Robert P, Gagneur G, Port M. Comparison of different types of blood pool agents (P792, MS325, USPIO) in a rabbit MR angiography-like protocol. Invest Radiol. 2003; 38: 311-9.

18. Goyen M, Shamsi K, Schoenberg SO. Vasovist-enhanced MR angiography. Eur Radiol. 2006; 16 Suppl 2: B9-14.

19. Lin W, Abendschein DR, Haacke EM. Contrast-enhanced magnetic resonance angiography of carotid arterial wall in pigs. J Magn Reson Imaging. 1997; 7: 183-90.

20. Adame IM, Van der Geest RJ, Wasserman BA, Mohamed MA, Reiber JHC, Lelieveldt BPF. Automatic segmentation and plaque characterization in atherosclerotic carotid artery MR images. Magma. 2004; 16: 227-34.

21. Roijers RB, Dutta RK, Cleutjens JP, Mutsaers PH, de Goeij JJ, van der Vusse GJ. Early Calcifications in Human Coronary Arteries As Determined with a Proton Microprobe. Anal Chem. 2008; 80: 55-61.

22. McCullagh P, Nelder J. Generalized linear models. 1989; London: Chapman and Hall.

23. Caravan P, Cloutier NJ, Greenfield MT, McDermid SA, Dunham SU, Bulte JW, Amedio JC Jr, Looby RJ, Supkowski RM, Horrocks WD Jr, McMurry TJ, Lauffer RB. The interaction of MS-325 with human serum albumin and its effect on proton relaxation rates. J Am Chem Soc. 2002; 124: 3152-62.

24. Nicholson JP, Wolmarans MR, Park GR. The role of albumin in critical illness. Br J Anaesth. 2000; 85: 599-610.

25. Smith EB, Staples EM. Distribution of plasma proteins across the human aortic wall: barrier functions of endothelium and internal elastic lamina. Atherosclerosis. 1980; 37: 579-90.

26. Barkhausen J, Ebert WJ, Heyer C, Debatin JF, Weinmann H. Detection of atherosclerotic plaque with Gadofluorine-enhanced magnetic resonance imaging. Circulation. 2003; 108: 605-9.

27. Zheng J, Ochoa E, Misselwitz B, Yang D, El Naqa I, Woodard PK, Abendschein D. Targeted contrast agent helps to monitor advanced plaque during progression: a magnetic resonance imaging study in rabbits. Invest Radiol. 2008; 43: 49-55.

28. Brushett C, Qiu B, Atalar E, Yang X. High-resolution MRI of deep-seated atherosclerotic arteries using motexafin gadolinium. J Magn Reson Imaging. 2008; 27: 246-50.

\section{ACKNOWLEDGEMENTS}

S.H. and M.D. participate in the European Vascular Genomics Network (http://www.evgn.org), a Network of Excellence supported by the European Community's Sixth Framework Program for Research Priority 1 (Life Sciences, Genomics, and Biotechnology for Health; contract LSHM-CT2003-503254). This workd was supported by a grant of the Dutch Heart Foundation (grant number 2002.B033) and the 'Besluit Subsidies Investeringen Kennisinfrastructuur (BSIK), entitled 'Molecular imaging of ischemic heart disease', project number BSIK 03033. 


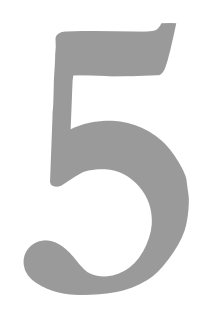

\section{Gadofosveset-enhanced magnetic resonance imaging of human carotid atherosclerotic plaques: initial experience}

M.B.I. Lobbes*, S. Heeneman*, V. Lima Passos, R. Welten, R.M. Kwee, R.J. van der Geest, A.J. Wiethoff, P. Caravan, B. Misselwitz, M.J.A.P. Daemen, J.M.A. van Engelshoven, T. Leiner**, M.E. Kooi** 


\section{ABSTRACT}

Background: Previously, we found that administration of gadofosveset resulted in a significantly higher signal enhancement on MR images of rabbit atherosclerotic vessel compared to healthy vessel walls. In the present study, the potential of MR imaging of human atherosclerotic plaques using gadofosveset was investigated.

Materials and methods: Fourteen (nine symptomatic, five asymptomatic) patients with carotid stenosis (70-99\%, according to NASCET criteria) were included. All subjects underwent baseline pre-contrast MR imaging. Immediately after completion of the baseline examination, $0.03 \mathrm{mmol} / \mathrm{kg}$ gadofosveset was administered. At 24 hours post-injection the acquisition was repeated. Twelve patients were scheduled for carotid endarterectomy. Carotid endarterectomy specimens were CD31-, CD68- and albumin-stained to correlate MR signal enhancement with intraplaque microvessel density, and macrophage and albumin content. In addition, the correlation between MR signal enhancement and size of various plaque components was assessed.

Results: We found variable signal enhancement amongst subjects. Subanalysis revealed that MR signal enhancement was significantly higher in symptomatic patients compared to asymptomatic patients $(1.72 \pm 0.07$ versus $0.88 \pm 0.03, \mathrm{p}<0.001)$. A significant correlation was found between MR signal enhancement and intraplaque albumin content $(p=0.01)$. There was no correlation between signal enhancement and microvessel density, macrophage content, area percentage of lipid-rich necrotic core, intraplaque hemorrhage, and fibrous tissue.

Conclusion: Post-gadofosveset signal enhancement was observed in carotid lesions of symptomatic patients. No enhancement was observed in asymptomatic patients. Enhancement was associated with differences in intraplaque albumin content. These findings warrant follow-up studies to evaluate the predictive value of post-gadofosveset MR signal enhancement for clinical events like stroke. 


\section{INTRODUCTION}

Patients with moderate to severe carotid atherosclerotic plaque are at risk for stroke, which increases with the degree of stenosis. Clinical trials showed that carotid endarterectomy in symptomatic patients with carotid artery stenosis of $70-99 \%$ is beneficial, whereas the beneficial effect of surgery in patients with a $30-69 \%$ stenosis is not clear [1]. A clear beneficial effect of surgery in the symptomatic patients with a $30-69 \%$ stenosis of the carotid artery might be found in a sub-group of patients who are at greater risk for subsequent stroke. It has been widely recognized that atherosclerotic plaque composition is important for the development of acute cerebrovascular events as certain plaques are prone to rupture leading to thromboembolic events. Characteristics of the high risk plaque are considered to be a large lipid-rich necrotic core, a thin fibrous cap, and a high inflammatory cell content [2]. Detection of these vulnerable plaques might be achieved with contrast-enhanced MR imaging [3,4]. Thus, there is a clear need for novel techniques that enable us to find a sub-group of patients with an increased risk for (recurrent) stroke.

MRI has the advantage of imaging both lumen diameter and vessel wall composition [3, 4]. Wasserman et al. demonstrated an improved contrast-tonoise ratio between fibrous tissue and lipid-rich necrotic core after administration of Gd-DTPA [5]. In other studies, contrast-enhanced MRI using Gd-DTPA enabled assessment of neovascularisation burden [6-9]. Until now, most studies used a non-specific gadolinium-containing contrast agent, such as Gd-DTPA. Recently, a new albumin-binding contrast agent, gadofosveset, became commercially available.

Gadofosveset (Vasovist $^{\circledR}$, MS-325, Bayer Schering Pharma AG, Berlin, Germany) is a blood pool agent that binds to serum albumin. It is currently used in magnetic resonance angiography (MRA) [10]. Albumin-binding results in a dramatic increase in both relaxivity and elimination half-life, providing a prolonged, significant MR signal enhancement even at low dose $[11,12]$.

Previously, we demonstrated that gadofosveset, but not Gd-DTPA, was able to discriminate between rabbit atherosclerotic and healthy control vessel wall [13]. A possible explanation for these results was the increased microvessel density in the atherosclerotic rabbit aorta vessel wall, enhancing the albumin content in the atherosclerotic vessel wall. In humans, the pres- 
ence of ('leaky') microvessels within the plaque is associated with plaque vulnerability [14]. Since albumin leaks into the plaque through microvessels, intraplaque albumin content might be associated with plaque vulnerability [15]. Therefore, gadofosveset-enhanced MR imaging of carotid artery stenosis might aid in the improved stratification of patients at risk of developing near-future stroke that may benefit most from surgical intervention or carotid stenting.

In the present study, we aimed to investigate the potential of gadofosvesetenhanced MR imaging for the assessment of human carotid atherosclerotic plaques. In addition (by correlating MR signal enhancement with area percentage of various plaque components, intraplaque microvessel density, macrophage and albumin content of the plaque) we sought more insight into post-gadofosveset MR signal enhancement in human carotid atherosclerotic plaques and evaluated whether there are differences in postgadofosveset signal enhancement between symptomatic and asymptomatic patients.

\section{MATERIALS AND METHODS}

\section{Subjects}

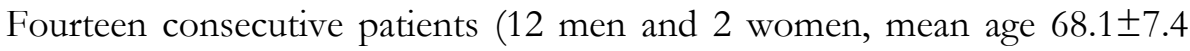
years, age range 54 to 76 years) with carotid atherosclerotic stenosis of 70 $99 \%$ (as demonstrated by duplex ultrasonography and assessed according to NASCET criteria) were recruited. In nine cases, carotid endarterectomy was indicated because of symptomatic carotid atherosclerosis, presenting as recent (within 3 months of MR imaging) TIA or minor stroke. In five cases, asymptomatic carotid atherosclerosis without prior neurological events was present. These patients were referred to the vascular surgeon because of a murmur over the carotid artery. Three of the five asymptomatic patients were diagnosed with progressive stenosis of the carotid arteries and therefore opted for surgery. The remaining two asymptomatic patients had stable carotid artery stenosis over time and did not choose for surgical intervention. Cerebral imaging (computed tomography or MRI) performed within two weeks before the pre-contrast MRI showed no signs of ischemic changes in all asymptomatic patients, confirming their asymptomatic status.

Prior to contrast administration, venous blood samples were drawn to assess renal function (expressed as the renal clearance in $\mathrm{mL} / \mathrm{min}$ according 
to Cockroft's formula). In twelve of the fourteen patients that were scheduled for carotid endarterectomy, surgery was performed within 5.9 \pm 3.2 days (range 3 to 12 days) after the post-contrast MRI scan. The study was approved by the institutional medical ethics committee. All participants gave written informed consent.

\section{MR imaging}

Immediately after baseline pre-contrast MR acquisition, patients received gadofosveset intravenously $(0.03 \mathrm{mmol} / \mathrm{kg}$ body weight). Based on the elimination rate of the compound and relaxivity data reported in human plasma, we chose a post-contrast imaging time of 24 hours [16].

Patient's heads were placed in a head holder to stabilize the head position. MR imaging was performed on a clinical 1.5T whole body MR scanner (Philips Healthcare, Best, The Netherlands) using a dedicated small diameter (47 $\mathrm{mm}$ ) radiofrequency surface coil that was attached to the neck at the location of the carotid bifurcation. A balanced, three-dimensional TFE sequence was performed to identify the carotid bifurcation using the following parameters: FOV $200 \times 200 \mathrm{~mm}$, matrix scan $192 \times 192$ (resulting in an inplane resolution of $1.04 \times 1.04 \mathrm{~mm}$ ), TR $4.4 \mathrm{~ms}$, TE $2.1 \mathrm{~ms}$, flip angle 60 degrees, truly acquired slice thickness $1.4 \mathrm{~mm}$, interpolated to $0.7 \mathrm{~mm}$, NSA 4. If necessary, the coil was repositioned to the position of the carotid bifurcation to optimize signal-to-noise ratio if necessary.

Subsequently, an ECG-triggered, T1-weighted, 2D DIR spin echo sequence was performed with the following scan parameters: 9 slices (partition thickness $3.0 \mathrm{~mm}$ with a gap of $0.5 \mathrm{~mm}$ ) positioned at the location of the atherosclerotic lesion, FOV $100 \times 80 \mathrm{~mm}$, matrix scan 256 x 192 (resulting in an in-plane resolution of $0.39 \times 0.39 \mathrm{~mm}$ ), NSA 2, flip angle 90 degrees, TR 1 heart beat, TE $18 \mathrm{~ms}$, and TSE factor of 9. TI was adjusted for cardiac frequency and $T_{1}$ relaxation time of blood. Before contrast administration, the $\mathrm{T}_{1}$ relaxation time of blood was assumed to be $1200 \mathrm{~ms}$, while the postcontrast $T_{1}$ relaxation time 24 hours after gadofosveset administration was assumed to be $515 \mathrm{~ms}$, based on the elimination rate of the compound and the relaxivity data reported in human plasma [16]. A CLEAR algorithm was used for image reconstruction to compensate for signal inhomogeneity. Total investigation time for a single MRI session was approximately $25 \mathrm{mi}-$ nutes. 


\section{MRI data analysis}

MR images were analyzed blinded for clinical status with VesselMASS software (Leiden University Medical Center, Leiden, the Netherlands). In the MR images of the T1-weighted DIR spin echo sequence, a ROI was drawn independently by two readers (M.L., R.K., 5 and 2 years of experience, respectively) encompassing the entire vessel wall [17]. ROI's were initially drawn in the post-contrast images. These ROI's were subsequently copied onto their corresponding pre-contrast images, allowing only minor modifications to adjust for positional changes. The mean signal intensity of this ROI was calculated and adjusted for gain and scaling factors. SE was defined as the quotient of post- and pre-contrast signal intensities. Assessment of MR image quality, pre- and post-contrast matching of MR images and matching with histological slices was performed by consensus reading of three readers (M.L., S.H., M.E.K.). To be included in the MRI analysis, both pre- and corresponding post-contrast images had to be of sufficient quality to enable delineation of the vessel wall contour. For analysis of the association between MR signal enhancement and intraplaque microvessel density, and macrophage/albumin content, an additional criterion was applied: the matching histological segment needed to be of sufficient quality to be analyzed. In the two patients not undergoing surgery, all MR images in which carotid plaque was observed were analyzed.

\section{Histology}

Immediately after removal by the vascular surgeon, the carotid endarterectomy specimen was cut into $3 \mathrm{~mm}$ thick slices, after which it was decalcified and formalin-fixed for 24 hours. Then, samples were processed and embedded in paraffin and $4 \mu \mathrm{m}$ thick (transverse) slices were cut and subsequently HE-stained. In addition, sections were immunohistochemically stained to assess intraplaque microvessel density, presence of macrophages, and intraplaque albumin content with anti-CD31 and anti-CD68 antibodies (clone JC70A and KP-1, respectively, Dako North America Inc., USA) and human serum anti-albumin antibody (AB8940, Abcam Inc., USA). Blinded for MR results, one reader (M.L.) assessed histological quality and sections of poor quality (i.e. sections which remained torn and/or distorted, or had a poorly recognizable tissue morphology, even after attempts of reprocessing the section) were excluded from analysis. 


\section{Histological analysis}

Digital photographs at a fixed magnifying field (125x) were acquired of all sections. Identification of the various plaque components (intraplaque hemorrhage, fibrous tissue, and lipid-rich necrotic core) was performed by consensus reading of two readers (M.L., S.H., 2 and 9 years of experience, respectively). Mixed tissue was defined as the collection of fibrous tissue, lipid-rich necrotic core and microvessels together, in case these plaque features could not be identified as separate areas. Plaque area and plaque components were measured by assessing the number of pixels within regions of interest drawn on the HE-stained sections using Image 1.40 (National Institute of Health, Bethesda, MD, USA). Sizes of different plaque components were expressed as percentages of the plaque area.

To assess intraplaque microvessel density within the histological sections, microvessels were counted in the hotspots of each section using 200x magnifying fields [18]. Intraplaque microvessels were defined as luminal structures surrounded by CD31-positive endothelial cells and were calculated as total CD31-positive microvessel count per magnifying field.

Macrophage and albumin content in the plaque were measured by quantifying the positive stained area on anti-CD68- and albumin-stained sections, respectively. The total area stained was divided by plaque area.

All immunohistochemical analyses were quantified using computerized morphometry (Leica QWin V3, United Kingdom) by one reader (M.L.), who was blinded for patient identification and MRI results.

\section{Statistical analysis}

All statistical analyses were performed with SPSS 15.0 (SPSS Inc, USA). Interobserver agreement of MR signal enhancement was calculated by determining the ICC. A non-parametric Mann Whitney $\mathrm{U}$ test was used to analyze differences between study groups concerning age, renal clearance, mean signal enhancement, and intraplaque albumin content. Significance of MR signal enhancement was tested with a one-sample t-test with the test value set at 1 (i.e. no signal enhancement). The relationship between MR signal enhancement and histological data (total plaque and plaque components area percentages, intraplaque microvessel density, and macrophage/albumin content) were evaluated by computing Pearson's correlation coefficient (r). Renal clearance, age, MR signal enhancement, total plaque 
area, and area percentages of various plaque components were presented as mean \pm standard error. All p-values $<0.05$ were considered statistically significant.

\section{RESULTS}

A total of $21 \mathrm{MR}$ image sets (i.e. matching pre- and post-contrast MR images) out of a total number of 112 image sets (19\%) from thirteen patients were excluded because of insufficient quality of the pre- or post-contrast image. Agreement between observers with regard to MR signal enhancement (as expressed by the ICC) was excellent: 0.956 ( $p<0.001)$.

There was a considerable variance in the magnitude of the signal enhancement observed in the different patients, varying from no apparent signal enhancement to strong signal enhancement (Figure 1). Mean signal enhancement was $1.37 \pm 0.07$ for all patients combined (range 0.46-3.80). Figure 2a shows box plots of both symptomatic and asymptomatic patients. Mean post-contrast signal intensity in symptomatic patients was increased significantly compared to the pre-contrast scan: $1.72 \pm 0.07$ (range 0.46-3.80, $\mathrm{p}<0.001)$. Mean post-contrast signal intensity in asymptomatic patients did not differ significantly pre-contrast signal intensity: $0.88 \pm 0.03$ (range 0.60 $1.43, \mathrm{p}=0.06)$. The difference in mean signal enhancement between symptomatic and asymptomatic patients was highly significant $(\mathrm{p}<0.001)$.

There was no significant difference in mean renal clearance or mean age between symptomatic and asymptomatic patients (Table 1). As a result of these findings, adjustment for possible confounding factors caused by differences in age or renal clearance of gadofosveset was not necessary. Figure $2 \mathrm{~b}$ shows typical MR images of a symptomatic and an asymptomatic patient.

Due to insufficient histological quality, $21 \%$ of the sections of 11 patients were excluded from analysis.

Histological analysis showed that there was no significant difference in plaque area between symptomatic and asymptomatic patients (21.6 versus 19.3 $\mathrm{mm}^{2}, \mathrm{p}=0.35$, Table 1). Symptomatic patients had a significantly higher amount of microvessels and macrophages (both $\mathrm{p}<0.001$, Table 1 ) when compared with asymptomatic patients. In addition, symptomatic patients had a significantly larger lipid-rich necrotic core when compared with asymptomatic patients $(p<0.001$, Table 1$)$, but there was no significant corre- 
lation between MR signal enhancement and total plaque area, area percentages of intraplaque hemorrhage, fibrous tissue, lipid-rich necrotic core, mixed tissue, intraplaque microvessel density, and macrophage content $(\mathrm{Ta}-$ ble 2). There was a weak, but significant, correlation between MR signal enhancement and percentage of plaque area positively stained for albumin $(\mathrm{r}=0.30, \mathrm{p}=0.01$, Table 2$)$.

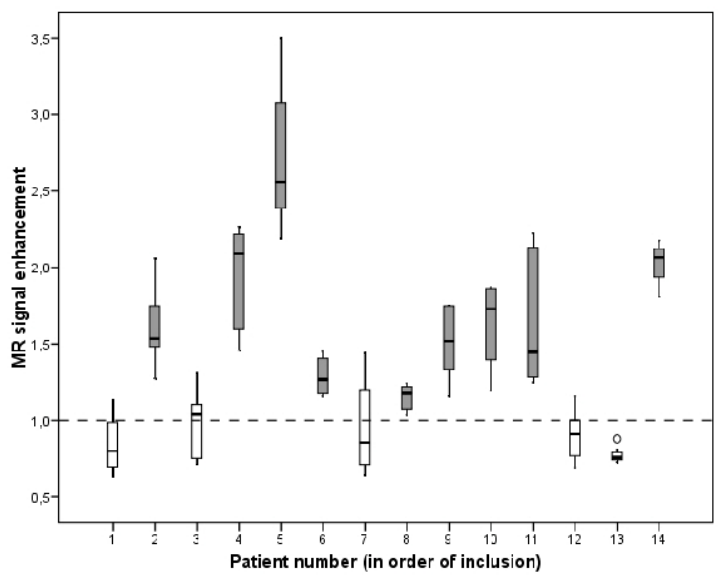

Figure 1. Box plots of observed signal enhancement of all individual patients in order of inclusion, clustering both symptomatic (grey) and asymptomatic (white) patients.

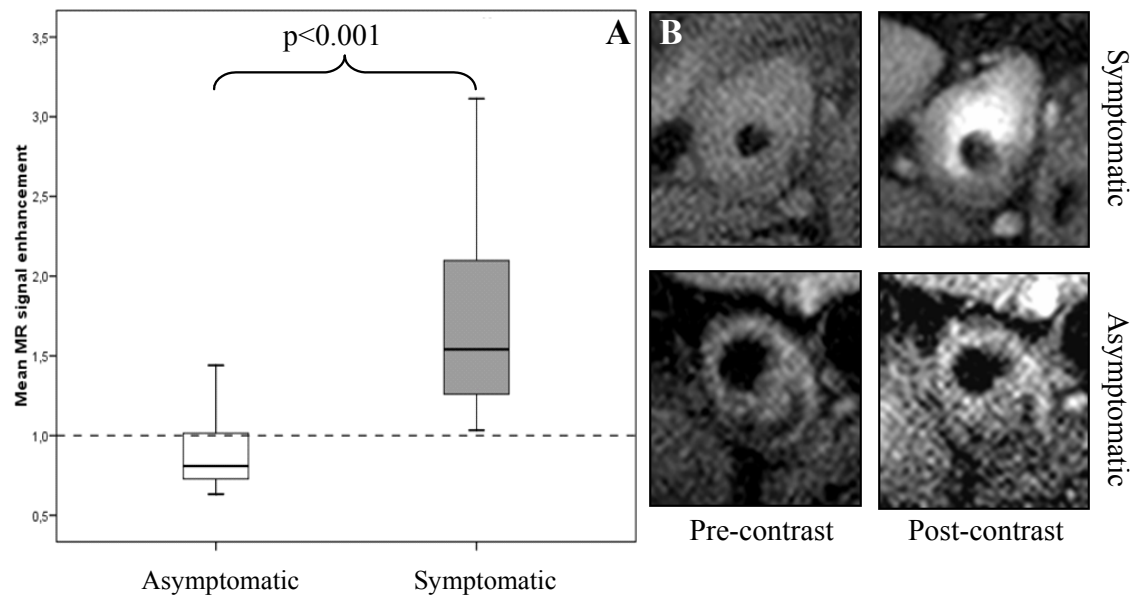

Figure 2. (A) Box plots of differences in mean MR signal enhancement between asymptomatic (white, $n=5$ ) and symptomatic (grey, $n=9$ ) patients. (B) Typical MR examples of pre- and post-contrast images of a symptomatic and asymptomatic patient. 


\section{DISCUSSION}

In the present study, we demonstrated a significantly higher MR signal enhancement 24 hours after administration of gadofosveset in symptomatic versus asymptomatic patients with carotid artery stenosis. However, there was no correlation between this MR signal enhancement and plaque size, area percentages of various plaque components, intraplaque microvessel density, or macrophage content. On the other hand, we demonstrated a weak but significant correlation between MR signal enhancement and intraplaque albumin content.

Table 1. Patient and plaque characteristics of both the symptomatic and asymptomatic study group. Renal clearance, age, and mean signal enhancement are presented as mean \pm standard error. ${ }^{*}$ Statistically significant.

\begin{tabular}{llll}
\hline Patient/plaque characteristics & Symptomatic & Asymptomatic & p-value \\
\hline Total number (male:female) & $9(7: 2)$ & $5(5: 0)$ & - \\
Renal clearance (mL/min) & $75 \pm 7$ & $90 \pm 9$ & 0.37 \\
Age (years) & $66 \pm 3$ & $65 \pm 2$ & 0.86 \\
Mean signal enhancement & $1.72 \pm 0.07$ & $0.88 \pm 0.03$ & $<0.001^{*}$ \\
Plaque area $\left(\mathrm{mm}^{2}\right)$ & $21.6 \pm 1.5$ & $19.3 \pm 3.6$ & 0.35 \\
Microvessel density (number) & $32 \pm 3$ & $12 \pm 3$ & $<0.001^{*}$ \\
Macrophage content $(\%)$ & $8.9 \pm 1.1$ & $2.7 \pm 0.4$ & $<0.001^{*}$ \\
Lipid-rich necrotic core $(\%)$ & $33.4 \pm 3.1$ & $10.0 \pm 4.8$ & $<0.001^{*}$ \\
Fibrous tissue $(\%)$ & $16.7 \pm 2.8$ & $24.5 \pm 4.8$ & 0.10 \\
Albumin content $(\%)$ & $44.4 \pm 3.2$ & $30.7 \pm 4.8$ & $0.05^{*}$ \\
\hline
\end{tabular}

Gadofosveset consists of a diphenylcyclohexyl group attached to a gadolinium chelate by a phosphodiester linkage. After intravenous administration, more than $75 \%$ of gadofosveset is (reversibly) bound to serum albumin. This results in an increased elimination half-life and an $r_{1}$ relaxivity in plasma that is approximately ten times higher at $1.5 \mathrm{~T}$ compared to other clinically approved gadolinium complexes [19, 20]. Its main clinical advantages are a prolonged window of opportunity for vascular imaging and higher post-contrast signal intensities at lower doses when compared with other, more commonly used Gd-chelates. Currently, gadofosveset is the only blood pool agent approved for MR angiography in the European Union and as such is mainly used for MR angiography studies [10].

In the current study, we observed a significant positive correlation between MR signal enhancement and intraplaque albumin content. Albumin is the 
most abundant protein present in the human circulation and leaves the circulation only through the fenestrated endothelium of capillaries [21]. Previously, we demonstrated a significant increase in post-gadofosveset MR signal enhancement after 11 hours in atherosclerotic rabbit vessel walls when compared with healthy vessel walls. In addition, there was a higher amount of albumin present within the atherosclerotic rabbit vessel walls compared to healthy vessel walls, whereas equal amounts of gadolinium atoms were present within vessel walls of atherosclerotic and healthy rabbits receiving gadofosveset [13]. In the same study we demonstrated an abundance of adventitial microvessels, suggesting that the vasa vasorum in the adventitia might be the main port of entry for albumin and gadofosveset [13]. The binding of albumin and gadofosveset within the plaque will result in a dramatic increase in $r_{1}$ relaxivity, leading to an intraplaque MR signal enhancement. In healthy rabbit vessels, considerably less albumin diffuses into the plaque, resulting in less increase in $r_{1}$ relaxivity of gadofosveset, leading to no apparent signal enhancement in the vessel wall.

Table 2. Association between MR signal enhancement and plaque components. *Data concern area percentages of plaque area. ${ }^{* *}$ Statistically significant.

\begin{tabular}{lll}
\hline Plaque components & r-value & p-value \\
\hline Plaque area & 0.180 & 0.145 \\
Fibrous tissue* & 0.160 & 0.185 \\
Lipid-rich necrotic core* & 0.004 & 0.970 \\
Mixed tissue* & 0.070 & 0.553 \\
Microvessel density* & 0.100 & 0.420 \\
Macrophage content* & 0.090 & 0.440 \\
Albumin content* & 0.300 & $0.010^{* *}$ \\
\hline
\end{tabular}

As suggested by our previous work, adventitial microvessels might be the port of entry of intraplaque albumin. Londono et al. studied the location of albumin within healthy human aortic tissue and demonstrated an uneven distribution of albumin over the vessel wall. Increasing levels of albumin were observed from lumen towards adventitia, with very high levels being observed around microvessels in the adventitia, the so-called vasa vasorum [22]. In addition, Fleiner et al. demonstrated in a post-mortem study that atherosclerotic plaques of iliac, renal, and carotid arteries in symptomatic patients contained a denser network of vasa vasorum compared to asymptomatic patients [23]. In the current study, microvessel density in the adventitia could unfortunately not be assessed as only the plaque and part of the media, that were removed during the carotid endarterectomy, were available 
for histological analyses. Thus, although differences in vasa vasorum density could explain the observed signal enhancement in the lesions of symptomatic patients, we were not able to investigate this further.

Intraplaque microvessel density was indeed higher in lesions of symptomatic patients compared to lesions of asymptomatic patients. This finding is in agreement with Mofidi et al., who demonstrated higher intraplaque microvessel counts in carotid endarterectomy specimens of symptomatic patients when compared with asymptomatic patients [24]. However, we did not observe a significant correlation between MR signal enhancement and intraplaque microvessel density, suggesting that the presence and amount of these intraplaque microvessels was no causative factor in the difference in signal enhancement between symptomatic and asymptomatic patients. An alternative explanation is the function and integrity of these microvessels. It has been suggested by Virmani et al., that rapid in-growth of microvessels from the adventitia into the plaque leads to abnormal vascular development of these vessels with disorganized branching, immature endothelial tubes, and 'leaky' imperfect linings [14]. Dunmore et al. demonstrated that within carotid artery plaques of symptomatic patients, irregular dysmorphic microvessels were found. These vessels lacked smooth muscle cells and were considered highly immature [25]. In addition, we recently studied intraplaque microvessel structure in normal and atherosclerotic coronary arteries. In contrast to our expectations, mural cell coverage was infrequent even in normal coronary arteries, and similar in all plaque types and vessel wall regions, including the adventitia. Thus, incomplete mural cell coverage cannot explain microvascular leakage in the coronary arteries. However, using electron microscopy, it was shown that endothelial junctions were aberrant or missing in intraplaque microvessels. Also, endothelial cell morphology frequently represented an activated, dysfunctional status and endothelial cells were frequently detached from the basal membrane of the microvessels. This compromised structural integrity of microvascular endothelium may thus explain microvascular leakage of compounds such as gadofosveset in atherosclerotic lesions [26]. In conclusion, we hypothesize that the increased MR signal enhancement observed in symptomatic patients might be caused by the increased amount of albumin within the plaques of symptomatic patients. Our working hypothesis is that an increased density of the vasa vasorum and/or compromised structural integrity of the endothelium of intraplaque microvessels, facilitated enrichment of albumin in symptomatic plaques, and consequently enabled intraplaque gadofosveset to increase its relaxivity, causing a detectable increase in signal intensity. In asymptomatic 
patients, less albumin entered the plaque via these routes, resulting in less increase in signal intensity.

Recently, Cornily et al. found a significant correlation between MR signal enhancement after administration of B22956/1 and intraplaque microvessel density in a rabbit model of atherosclerosis [27]. B22956/1 is a novel intravascular contrast agent which (like gadofosveset) has a high affinity for serum albumin. Their results were in line with the result of our previous rabbit study [13]. In addition, they also demonstrated a significant correlation between signal enhancement and intraplaque macrophage content. In the current study, we did not find a significant correlation between signal enhancement and macrophage content when using gadofosveset (Table 1). This might be explained by differences between human and rabbit atherosclerotic plaques or differences in uptake between these two contrast agents. Unlike gadofosveset, which has been registered for human MR angiography studies in Europe, B22956/1 has not been registered for human applications.

Identification of 'true' asymptomatic patients remains a topic of discussion. We defined the asymptomatic patient as a patient without prior neurologic event. This was based on documented patient history and confirmed during patient interview prior to MRI acquisitions. Absence of previous cerebrovascular events was additionally assessed with additional dedicated cerebral CT or MRI to determine the presence of prior (asymptomatic) stroke. With this approach, we minimalized the chance of missing a recent ischemic event in the asymptomatic study group.

Our preliminary findings presented in this study can have important clinical consequences. According to pooled analysese of several large trials (including NASCET and ECST), patients with symptomatic carotid artery disease and a stenosis of more than $70 \%$ (as demonstrated on Duplex ultrasonography) benefit from carotid surgery $[1,28]$. However, for the group of patients with stenosis of $30-69 \%$ it is still not clear whether they benefit from surgical intervention or carotid stenting [1]. Gadofosveset-enhanced MR imaging of human carotid artery atherosclerosis might aid in the improved identification of people who are at risk of developing near-future stroke, but who have a carotid artery stenosis of less than $70 \%$. In addition, postgadofosveset signal enhancement might be associated with adverse outcome in this population. Based on our initial results, future studies are warranted that investigate the potential of gadofosveset-enhanced MR imaging of ca- 
rotid artery atherosclerosis and whether MR signal enhancement after gadofosveset administration might have a prognostic value in predicting future cerebrovascular events.

In conclusion, we demonstrated the potential of gadofosveset-enhanced carotid plaque MR imaging as mean MR signal enhancement was significantly higher in symptomatic patients with carotid artery atherosclerosis when compared with asymptomatic patients. Based on our histological analyses, we hypothesized that increased presence of intraplaque albumin in symptomatic patients, which might enter the plaque through adventitial and intraplaque microvessels, played a major role in this phenomenon. 


\section{REFERENCES}

1. Rothwell PM, Eliasziw M, Gutnikov SA, Fox AJ, Taylor DW, Mayberg MR, Warlow CP, Barnett HJ, Carotid Endarterectomy Trialist's Collaboration. Analyses of pooled data from the randomised controlled trials of endarterectomy for symptomatic carotid stenosis. Lancet. 2003;361:107-16.

2. Lusis AJ. Atherosclerosis. Nature. 2000;407:233-41.

3. Sanz J, Fayad ZA. Imaging of atherosclerotic cardiovascular disease. Nature. 2008;451:953-7.

4. Yuan C, Kerwin WS. MRI of atherosclerosis. J Magn Reson Imaging. 2004;19:710-9.

5. Wasserman BA, Smith WI, Trout HH 3rd, Cannon RO 3rd, Balaban RS, Arai AE. Carotid artery atherosclerosis: in vivo morphologic characterization with gadoliniumenhanced double-oblique MR imaging initial results. Radiology. 2002;223:566-73.

6. Kerwin W, Hooker A, Spilker M, Vicini P, Ferguson M, Hatsukami T, Yuan C. Quantitative magnetic resonance imaging analysis of neovasculature volume in carotid atherosclerotic plaque. Circulation. 2003;107:851-6.

7. Kerwin WS, O'Brien KD, Ferguson MS, Polissar N, Hatsukami TS, Yuan C. Inflammation in carotid atherosclerotic plaque: a dynamic contrast-enhanced MR imaging study. Radiology. 2006;241:459-68.

8. Kerwin WS, Oikawa M, Yuan C, Jarvik GP, Hatsukami TS. MR imaging of adventitial vasa vasorum in carotid atherosclerosis. Magn Reson Med. 2008;59:507-14.

9. Lin W, Abendschein DR, Haacke EM. Contrast-enhanced magnetic resonance angiography of carotid arterial wall in pigs. J Magn Reson Imaging. 1997;7:183-90.

10. Goyen M, Shamsi K, Schoenberg SO. Vasovist-enhanced MR angiography. Eur Radiol. 2006; 16 Suppl 2:B9-14.

11. Corot C, Violas X, Robert P, Gagneur G, Port M. Comparison of different types of blood pool agents (P792, MS325, USPIO) in a rabbit MR angiography-like protocol. Invest Radiol. 2003;38:311-9.

12. Caravan P, Cloutier NJ, Greenfield MT, McDermid SA, Dunham SU, Bulte JW, Amedio JC Jr, Looby RJ, Supkowski RM, Horrocks WD Jr, McMurry TJ, Lauffer RB. The interaction of MS-325 with human serum albumin and its effect on proton relaxation rates. J Am Chem Soc. 2002;124:3152-62.

13. Lobbes MBI, Miserus RJJHM, Heeneman S, Lima Passos V, Mutsaers PHA, Debernardi N, Misselwitz B, Post M, Daemen MJAP, Van Engelshoven JMA, Leiner T, Kooi ME. Contrast-enhanced magnetic resonance vessel wall imaging in a rabbit model of atherosclerosis: comparison of gadofosveset and Gd-DTPA. Radiology. 2009; In press.

14. Virmani R, Kolodgie FD, Burke AP, Finn AV, Gold HK, Tulenko TN, Wrenn SP, Narula J. Atherosclerotic plaque progression and vulnerability to rupture: angiogenesis as a source of intraplaque hemorrhage. Arterioscler Thromb Vasc Biol. 2005;25:54-61.

15. Zhang Y, Cliff WJ, Schoefl GI, Higgins G. Immunohistochemical study of intimal microvessels in coronary atherosclerosis. Am J Pathol. 1993;143:164-72.

16. Eldredge HB, Spiller M, Chasse JM, Greenwood MT, Caravan P. Species dependence of plasma protein binding and relaxivity of the gadolinium-based MRI contrast agent MS325. Invest Radiol. 2006;41:229-43.

17. Adame IM, Van der Geest RJ, Wasserman BA, Mohamed MA, Reiber JH, Lelieveldt BP. Automatic segmentation and plaque characterization in atherosclerotic carotid artery MR images. MAGMA. 2004;16:227-34.

18. Weidner N. Current pathologic methods for measuring intratumoral microvessel density within breast carcinoma and other solid tumors. Breast Cancer Res Treat. 1995;36:169-80. 
19. Parmelee DJ, Walovitch RC, Ouellet HS, Lauffer RB. Preclinical evaluation of the pharmacokinetics, biodistribution, and elimination of MS-325, a blood pool agent for magnetic resonance imaging. Invest Radiol. 1997;32:741-7.

20. Lauffer RB, Parmelee DJ, Dunham SU, Ouellet HS, Dolan RP, Witte S, McMurry TJ, Walovitch RC. MS-325: albumin-targeted contrast agent for MR angiography. Radiology. 1998;207:529-38.

21. Nicholson JP, Wolmarans MR, Park GR. The role of albumin in critical illness. Br J Anaesth. 2000;85:599-610.

22. Londono I, Leclerc Y, Bendayan M. Ultrastructural localization of endogenous albumin in human aortic tissue by protein A-gold immunocytochemistry. Am J Pathol. 1992;140:179-91.

23. Fleiner M, Kummer M, Mirlacher M, Sauter G, Cathomas G, Krapf R, Biedermann BC. Arterial neovascularization and inflammation in vulnerable patients: early and late signs of symptomatic atherosclerosis. Circulation. 2004;110:2843-50.

24. Mofidi R, Powell TI, Crotty T, Mehigan D, Macerlaine D, Keaveny TV. Angiogenesis in carotid atherosclerotic lesions is associated with timing of ischemic neurological events and presence of computed tomographic cerebral infarction in the ipsilateral cerebral hemisphere. Ann Vasc Surg. 2008;22:266-72.

25. Dunmore BJ, McCarthy MJ, Naylor AR, Brindle NP. Carotid plaque instability and ischemic symptoms are linked to immaturity of microvessels within plaques. J Vasc Surg. 2007;45:155-9.

26. Sluimer JC, Kolodgie F, Bijnens AP, Pacheco E, Duimel H, Frederik PM, Van Hinsbergh VWM, Virmani R, Daemen M. Infrequent pericyte coverage and clear structural abnormalities in intraplaque microvessels in human coronary atherosclerotic plaques. JACC. 2009; In press.

27. Cornily JC, Hyafil F, Calcagno C, Briley-Saebo KC, Tunstead J, Aguinaldo JG, Mani V, Lorusso V, Cavagna FM, Fayad ZA. Evaluation of neovessels in atherosclerotic plaques of rabbits using an albumin-binding intravascular contrast agent and MRI. J Magn Reson Imaging. 2008;27:1406-11.

28. Cina CS, Clase CM, Haynes RB. Carotid endarterectomy for symptomatic carotid stenosis. Cochrane Database Syst Rev. 2000(2):CD001081.

\section{ACKNOWLEDGEMENTS}

We wish to acknowledge Anique Jansen-Vrehen for her assistance in optimizing and performing the immunohistochemical stainings presented in this study.

S.H. and M.D. participate in the European Vascular Genomics Network (http://www.evgn.org), a Network of Excellence supported by the European Community's Sixth Framework Program for Research Priority 1 (Life Sciences, Genomics, and Biotechnology for Health; contract LSHM-CT2003-503254). This work was supported by a grant of the Dutch Heart Foundation (grant number 2002.B033) and the 'Besluit Subsidies Investeringen Kennisinfrastructuur (BSIK), entitled 'Molecular imaging of ischemic heart disease', project number BSIK 03033. 


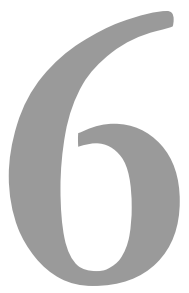

General discussion 
Atherosclerosis is a chronic inflammatory disease of large arteries that is characterized by the accumulation of lipids and inflammatory components in the vessel wall, resulting in the development of atherosclerotic plaques [13]. These plaques can eventually rupture, leading to clinical events such as myocardial infarction and stroke [4].

Several risk assessment models for atherosclerosis have been developed over the past decades $[5,6]$. Common determinants of atherosclerotic disease, such as age, hypertension, smoking, diabetes, and hyperlipidemia are included in these models. Approximately $20 \%$ of the cases of incidental myocardial infarction or ischemic stroke occur in individuals with few to none of these risk factors. In addition, $50 \%$ of incidental myocardial infarction cases occur in patients without hyperlipidemia [7]. Therefore, there is a large interest in other ways to identify patients who are at high risk of developing near-future cardiovascular disease. A roadmap to identify patients at high risk of developing near-future cardiovascular complications was suggested [8-10]. In this roadmap, individuals of the general population who have an increased risk of near-future cardiovascular events are identified with the use of questionnaires and biomarker profiling. Next, these high-risk individuals can be further evaluated using non-invasive (molecular) imaging. In the present thesis, we focused on two aspects of this roadmap, namely non-invasive risk assessment, i.e. validation of (potential) biomarkers, and on magnetic resonance imaging to visualize and characterize atherosclerotic plaques.

With respect to the validation of potential biomarkers, we performed a systematic review that showed that the prognostic value of SCD40L, IL-6, and oxLDL in predicting cardiovascular disease was comparable with the prognostic value of well-established biomarkers, such as CRP and fibrinogen (chapter 2). In a second study, we demonstrated that leukocyte counts and levels of fibrinogen, CRP, MPO, and PAPP-A were increased in patients with acute coronary syndromes. When combined, leukocyte counts, MPO, and PAPP-A predicted study group (acute versus non-acute coronary syndromes) correctly in $83 \%$ of the cases (chapter 3 ).

With respect to MRI of atherosclerotic plaques, we investigated the potential of contrast-enhanced plaque imaging in both an atherosclerotic rabbit model and human carotid artery atherosclerosis (chapters 4 and 5, respectively). For this purpose, we used the blood pool contrast agent gadofosveset, which is able to reversibly bind to serum albumin after intravenous ad- 
ministration, leading to increased $\mathrm{r}_{1}$ relaxivity and elimination half-life. In the rabbit study, we demonstrated that gadofosveset showed specific MR signal enhancement in the atherosclerotic vessel wall, whereas Gd-DTPA could not discriminate between normal and atherosclerotic rabbit vessel wall. In a subsequent human study, we demonstrated that gadofosveset administration resulted in significant post-contrast signal enhancement of carotid plaques of symptomatic patients when compared with carotid plaques of asymptomatic patients (chapter 5).

\section{BIOMARKERS OF CARDIOVASCULAR DISEASE}

Biomarkers are biochemical features that can be used to measure the disease course or the effect of treatment [11]. Genomic and proteomic research on the characteristics of atherogenesis and plaque rupture with thrombus formation resulted in the identification of several circulating biomarkers that may predict future cardiovascular events or outcome [12]. These biomarkers are mainly parameters of lipid burden (oxLDL), hemostasis and/or thrombosis (fibrin, plasminogen-activator inhibitor-1, and D-dimers), neurohormone activation (renin and BNP), insulin resistance (insulin and haemoglobin A1C), endothelial dysfunction (homocysteine and urinary albumin) and inflammation (CRP, sCD40L, MPO, and IL-6) [13].

\section{The five phases of biomarker development}

The process of biomarker development can be summarized in roughly five phases (Figure 1) [14]. The phases of research are ordered according to the strength of evidence that each provides in favor of the biomarker (from weakest to strongest) and results of earlier phases are required to design later phases.

The first phase aims to identify and prioritize potential new biomarkers. The primary aim of the second phase is to evaluate the biomarker assay's ability to distinguish between diseased and healthy subjects. The third phase focuses on the capacity of the biomarker to detect the disease in a preclinical stage. Longitudinal retrospective studies, for example NCC, can be used for this purpose. In addition, this phase should define criteria for a 'positive screening'. Phase four uses prospective studies in which definitive diagnostic procedures or treatments are applied in subjects that fulfill the criteria of being 'positive'. Therefore, phase four gives an impression on the number and nature of the subjects that screened positive, but it additionally gives 
information on false-positives. Finally, phase five estimates reductions in disease mortality and/or morbidity as a result of the screening test.

PHASE 1

PHASE 2

PHASE 3

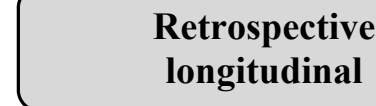

PHASE 4

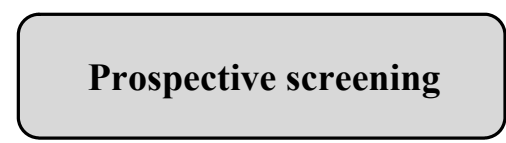

PHASE 5

Clinical assay and validation
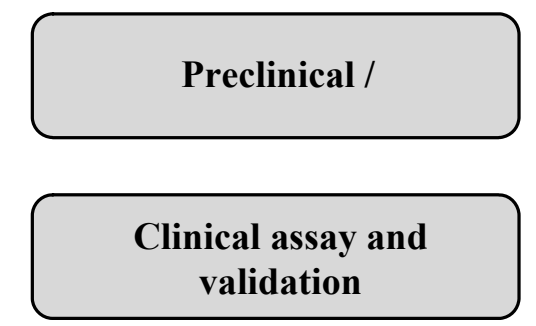

the assay's ability to distinguish between diseased and healthy subjects

Capacity of the biomarker to detect disease in preclinical stage / define criteria for positive screening

Definitive diagnostic procedures and treatments are applied in 'positive' subjects

Estimates reduction is disease mortality and/or morbidity as a result of the screening test

Figure 1. The five phases of biomarker development.

\section{$V$ alidation of established biomarkers of cardiovascular disease}

At the start of this thesis, only two serum biomarkers were validated extensively and had shown a potential for future clinical use: CRP and fibrinogen.

Ridker et al. showed that in apparently healthy men and women, increased plasma levels of CRP were a strong independent predictor of the development of future cardiovascular disease [15]. In a prospective nested casecontrol study, Tracy et al. demonstrated that CRP was associated with cardiovascular events (such as myocardial infarction, angina pectoris, and death) in healthy elderly men and women, especially in cases with subclinical cardiovascular disease at baseline [16]. Morrow et al. demonstrated a predictive value of CRP for short-term mortality in a cohort of patients with unstable angina pectoris and non-Q-wave myocardial infarction in combination with negative cardiac specific troponin $\mathrm{T}$ (mortality rate of $5.80 \%$ with increased levels of CRP versus $0.36 \%$ for low levels of CRP) [17]. Recently, Arima et al. showed in a general Japanese population that increased levels of 
CRP resulted in a threefold increase in risk of developing coronary heart disease within fourteen years [18]. Over the years, the potential predictive value of CRP for cardiovascular events have been confirmed in men and women, smokers and non-smokers, and diabetic and non-diabetic patients [19].

Elevated concentrations of fibrinogen have been associated with atherosclerosis and cardiovascular disease in patients with carotid stenosis and coronary artery disease, and its predictive value for future cardiovascular events has been proven in many studies [20]. For example, Maresca et al. showed in a meta-analysis of twenty-two studies that the overall risk estimate of cardiovascular events in patients with elevated concentrations of fibrinogen were twice as high as in patients with low concentrations of fibrinogen (OR 1.99) [21]. Stec et al. showed that in 2,632 subjects of the Framingham Offspring Study fibrinogen levels of those with cardiovascular disease were significantly higher than those without cardiovascular disease [22]. Baker et al. demonstrated in a population of 4,860 men and a follow-up period of 15 to 19 years that men who developed ischemic heart disease had significantly higher concentrations of fibrinogen than disease-free individuals. However, no significant difference in fibrinogen levels could be observed between disease-free men and men that developed ischemic stroke [23].

These positive results on CRP and fibrinogen do not imply that they are the 'Holy Grail' of cardiovascular biomarkers. For instance, all of these studies present a relative risk estimate based on the analysis of a large population. They do not represent the increased risk of an individual patient. It also remains unclear whether an elevated concentration of CRP is a risk factor on its own, or if it is a marker of other risk factors that are already present within the patient. In addition, there is no evidence that reducing elevated levels of CRP reduces the risk of developing cardiovascular events, although studies showed that statin use decreases both CRP levels and the occurrence of cardiovascular events [24]. Furthermore, it was recently shown in a metaanalysis of 31 studies covering the period 1963-2003 that fibrinogen levels increase with age and that it shows approximately linear associations with other cardiovascular risk markers. One third of the variation could be explained by cohort, age and sex. Additional variation (7\%) could be explained by established risk factors such as smoking and body mass index. A further $10 \%$ of the variation was explained by other inflammatory markers, mostly CRP. Finally, the association with body mass index was twice as strong in women compared to men, while the association with smoking was stronger 
in men [25]. These factors could all influence concentrations of CRP and/or fibrinogen. Thus, these findings raise doubts about the predictive value of CRP and fibrinogen for an individual patient and the causality of these biomarkers in cardiovascular complications. Study results on the predictive value of these biomarkers in cardiovascular disease should be interpreted with these limitations in mind.

\section{There is more than CRP and fibrinogen!}

In the current thesis we therefore aimed to assess the relative risk estimates of biomarkers other than CRP and fibrinogen, to evaluate their potential to become a future biomarker of cardiovascular disease. With this respect, we decided to search the available literature for the prognostic value of cardiovascular disease for soluble sCD40L, IL-6, and oxLDL. All of these markers had proven to be involved in atherosclerosis in in vitro, and in vivo animal and human studies [26-31].

In a systematic review we investigated the predictive value of sCD40L, IL-6, and oxLDL for cardiovascular disease (chapter 2). Our main conclusion was that elevated concentrations of these three biomarkers were associated with increased risk of developing cardiovascular disease. However, the number of included studies was only limited (4 for sCD40L, 10 for IL-6, and 2 for oxLDL), despite the large number of hits we acquired during our initial literature search. This was explained by the fact that a large part of the studies were cross-sectional, determining serum concentrations of the respective biomarkers at baseline and investigating their association with the presence or absence of cardiovascular disease. Only few prospective studies were performed for each of the biomarkers. These studies presented the predictive value of these biomarkers in a certain population of interest, but the increased relative risk estimation for a single patient cannot be given by this study design. Another major drawback that we encountered during this systematic review was the fact that study designs of these prospective studies differed tremendously, hampering the pooling of data into a metaanalysis. In this review, we emphasized the need for more and larger prospective studies for each of the biomarkers investigated. But even more important, we stressed the need for similar study designs, enabling pooling of available data for determining the true potential prognostic value of a single biomarker. In our opinion, NCC studies are ideal cohorts for the initial validation of potential new biomarkers. NCC studies allow for rapid analysis of large numbers of both patients and disease free controls. In many cases, these studies are running for several years, usually withdrawing 
sufficient amounts of blood for additional laboratory analyses, ensuring that blood samples are (often) already available. However, the inclusion of large populations is labor intensive and costly. In this setting, the association and prognostic value of biomarkers can be retrospectively assessed for a large population during a long period of follow-up. Future studies on biomarker validation should use NCC studies and comparable designs to allow rapid assessment and pooling of data.

Laboratory assays to determine concentrations of soluble CD40L, IL-6 and oxLDL are commercially available and user-friendly, allowing assessment of these biomarkers outside of experimental settings. Their relative risk estimates are comparable to CRP and fibrinogen, making them equally potential in predicting future adverse cardiovascular events. They should be considered if a so-called 'multi-marker approach' is to be used in future studies to predict future cardiovascular risk. In a multi-marker approach, the prognostic value for cardiovascular disease of a panel of markers combined is assessed [32]. The advantages and disadvantages of this approach will be discussed in a following paragraph.

\section{Identification of new and emerging biomarkers}

The predictive value of current known established markers has proven to be moderate at best. Therefore, there is a continuing search for new biomarkers of atherosclerosis, which might have more clinical impact than the established biomarkers.

Technological improvements, such as high-throughput genomics and proteomics and phage display, are expected to enable the identification of potential new biomarkers for cardiovascular disease in the first phase of biomarker development. With these promising techniques, large quantities of potential new biomarkers for a certain disease can be identified. However, this is also a disadvantage, since all of these biomarkers should be further evaluated for their prognostic value and many of these biomarkers will prove to be unsuitable for clinical use. Another drawback of these techniques is that they are very time-consuming.

High-throughput genomic and proteomic approaches and sensitive assays for determining marker concentration have lead to an expansive growth of possible biomarkers for cardiovascular disease. For example, Tabibiazar et al. were able to identify upregulation of multiple inflammatory markers in atherosclerotic mice using microarray analysis. Several chemokines (e.g. 
CCL2, CCL9, CCL11), cytokines (e.g. IL-2, IL-5, IL-6), and other inflammatory proteins (e.g. Csf1, Csf2, Ifng) were upregulated in these mice compared to control mice [33]. Choi et al. evaluated the serum levels of the adipokine lipocalin-2 (as determined by custom made immunoassays) with conventional cardiovascular risk factors and demonstrated increased serum levels and an independent association in patients with coronary heart disease [34]. In patients with coronary artery disease, Shlipak et al. demonstrated that increased serum levels of N-terminal prohormone BNP (as determined by a specific N-terminal prohormone BNP assay) were associated with increased risk (hazard ratio of 2.1) of developing the composite outcome stroke/myocardial infarction/death due to coronary artery disease within 3.5 years [35]. Kraaijeveld et al. demonstrated increased plasma levels of CCL5 (32.7 versus $23.1 \mathrm{ng} / \mathrm{ml}$ in stabilized patients) and CCL18 (104.4 versus $53.7 \mathrm{ng} / \mathrm{ml}$ ) in patients with UAP. Additionally, it was demonstrated that increased levels of CCL5 and CCL18 predicted future cardiovascular events. Elevated levels of CCL18 were slightly more predictive than those of CCL5, as indicated by the receiver operating characteristic curve (area under the curve 0.71 versus 0.69 ) [36]. Tuomainen et al. analyzed the association between cardiovascular disease or subclinical atherosclerosis during a follow-up period of ten years and showed an increased risk for cardiovascular disease in healthy men with increased serum MMP8 levels (relative risk 1.2) [37]. In all of the above studies, phase one of the biomarker development process was completed. In addition, all studies (except for the study by Tabibiazar) performed a phase two study, in which the biomarker assay's ability to distinguish between diseased and healthy individuals was evaluated. For three studies (by Shlipak et al., Kraaijeveld et al., and Tuomainen et al.) phase three of the biomarker development process was completed, in which they tested the capacity of the biomarkers to detect preclinical disease. However, they did not discuss the criteria of the specific biomarker for positive screening (i.e. the criteria needed for a patient to be 'positive') and no additional phases were completed until now. The potential of novel biomarkers for predicting future cardiovascular disease needs to be validated not only in small (phase one and two) studies, but also in large retrospective (phase three) and prospective (phase four) longitudinal studies and in different patient populations. Due to the availability of large cohorts, biomarker testing can be performed relatively easily up to phase three, in which biomarkers are tested in a NCC study design. The next step, phase four, requires prospective study designs and it will take time and resources to complete these studies. The development of adequate biomarkers is therefore delayed in phase four, making it less desirable for research departments to 
invest time and money in these final phases of biomarker development. Performing meta-analyses of individual participant data from multiple small cohorts might circumvent this problem [13]. However, for a biomarker to become clinically applicable, all phases have to be successfully completed.

In conclusion, current 'new' biomarkers have completed the biomarker development process up to a maximum of phase three. It is essential for these biomarkers to take the next step and complete phases four and five. Nonetheless, clinical application of these biomarkers will still take some years to become reality, since completion of the additional phases takes time.

Potential new biomarkers can also be identified by analyzing the culture medium in which the specimen of interest, e.g. an atherosclerotic plaque, is placed: the secretome. The secretome is the population of genes and/or proteins that is secreted from the tissue of interest, e.g. the atherosclerotic plaque. By combining two-dimension gel electrophoresis and mass spectrometry, Duran et al. demonstrated that two isoforms of HSP27 were upregulated in human atherosclerotic plaque secretomes, even though the exact role of HSP27 in atherogenesis is still largely unknown [38]. In another study, Duran et al. assessed the secretome of cultured atherosclerotic plaques in the presence of atorvastatin. This secretome analysis showed upregulation of 24 proteins and downregulation of 20 proteins compared with controls. Some of these proteins, such as (the upregulated) cathepsin $\mathrm{D}$, might have a potential role in plaque progression, making it a potential biomarker for assessing cardiovascular risk [39]. These findings proved that with analysis of the secretome, it was possible to identify potential new biomarkers for the disease of interest. However, these studies did not report an assay capable of measuring biomarker concentrations and therefore were not able to demonstrate that these biomarkers were able to distinguish between diseased and healthy individuals.

Phage display techniques can also aid in the identification of potential new biomarkers. Phage display is defined as 'a technique in which bacteriophages are engineered to produce and expose or display a foreign peptide fused to one of their capsid proteins at the phage's external surface. The phage can then be used to screen which ligand, antibody or antigen binds to the expressed fusion protein' [40]. By using serological antigen screening, Cleutjens et al. were able to identify two (as yet unidentified) antibodies against cDNA products that were subsequently evaluated on serum samples of a 
cohort of patients with peripheral atherosclerotic lesions $(n=75)$, different stages of coronary artery disease $(n=99)$, and healthy controls $(n=40)$ [40]. In serum, the identified set antibodies showed a specificity and sensitivity of $100 \%$ and $76 \%$, respectively, for discriminating patients with PAD. In addition, $93 \%$ of the patients with AMI tested positive for these biomarkers, while all patients with SAP tested negative. Although these findings are promising, the next step in validating these biomarkers will be their evaluation in a retrospective study to assess their ability to detect preclinical disease and additionally, criteria for positive screening should be formulated.

\section{$V$ alidation of new and emerging biomarkers}

The ability of potential new biomarkers' assays to distinguish between diseased and healthy individuals should be evaluated in phase two of biomarker development. Examples of assays that can be used for this purpose are Western blotting, ELISA, and immunohistochemistry. If these assays show favorable results, the biomarker can be tested in phase three studies, in which the potential of the biomarkers can be further validated and analyzed in retrospective longitudinal studies of well-defined populations [41]. This approach was used by Wilson et al., who used proteomic profiling of plasma samples to search for novel biomarkers of PAD. They demonstrated that B2M was upregulated in PAD patients compared to patients with no $\mathrm{PAD}$ and therefore, they proposed B2M as a biomarker for PAD. Subsequently, validation of these findings was performed in a clinical study with PAD versus non-PAD patients by determining B2M with ELISA. B2M was elevated in patients with PAD and correlated with the severity of disease, independent of other risk factors for PAD [42]. Nonetheless, retrospective or prospective (phase three or four of the biomarker development process) studies with $\mathrm{B} 2 \mathrm{M}$ in $\mathrm{PAD}$ are yet to be reported.

With respect to this thesis, we investigated the potential of new, emerging biomarkers, such as MPO. This study was a phase two study in the process of biomarker development, showing the ability of the (commercially available) assay kits to distinguish between diseased and healthy participants. The next step would be to perform a retrospective longitudinal study to assess whether these biomarkers would be able to detect disease in a preclinical stage. With these results, one could define criteria for positive screening, after which a prospective study could be performed in which diagnostic procedures are applied in subjects that screened positive on our biomarkers. Finally, if results are favorable, estimates on mortality/morbidity reduction 
when these biomarkers are applied could be made to complete the process development of myeloperoxidase as a biomarker.

However, the presented study constituted of only a small population size $(\mathrm{n}=120)$ in which only a limited number of emerging biomarkers was investigated (MPO and PAPP-A). Nonetheless, this study directly compared established and emerging markers in a population consisting not of one, but three cardiovascular diseases and compared them with healthy controls (all groups $n=30)$. In a discriminant analysis, we demonstrated that leukocyte counts, MPO, and PAPP-A had the most potential as biomarkers for cardiovascular disease. This suggestion could not be further evaluated, since our small population size and limited follow-up (of 6 months) resulted in too few adverse events to accurately assess the predictive value of the (combination of) biomarkers as expressed as a relative risk estimate.

It is currently possible to determine extremely low serum or plasma concentrations (up to nanograms per liter) of biomarkers. However, dedicated or custom made laboratory assays, mainly ELISA, are needed to determine these concentrations. Since many biomarkers are still under investigation, laboratory assays of these markers are usually custom made (especially when biomarkers are 'new'), which hampers the assessment of (initial) study results and application of the biomarker in a clinical setting. The use of custom made assays might result in favorable results, but differences in assays used can lead to conflicting results.

For example, positive immunohistochemical staining for PAPP-A in atherosclerotic plaques and an elevation of serum PAPP-A levels in acute coronary syndromes was demonstrated by Bayes-Genis et al. [43]. However, these results could not be reproduced by Dominguez-Rodriguez et al. when they used a custom available kit for the assessment of PAPP-A [44]. In line with the results from the latter publication, we also did not find any differences in PAPP-A concentrations in patients with stable and unstable angina pectoris, myocardial infarction, and healthy controls when we used the similar commercially available kit from DRG Laboratories (chapter 3). The discrepancy is thought to be due to the differences in the characteristics of serum PAPP-A. In healthy controls, PAPP-A was present in both a complexed and an uncomplexed form with proMBP. It was the uncomplexed PAPP-A that originated from atherosclerotic lesions and which was most likely determined by the custom made kits previously reported by Qin et al. [45]. However, commercial kits determine the total amount of PAPP-A, since 
they are originally designed to demonstrate the presence of Down's syndrome during pregnancy. With an additional ultrasensitive research kit (PAPP-A US) we demonstrated that in the same study population, levels of PAPP-A were significantly increased in patients with AMI when compared to the other study groups (chapter 3). This example emphasizes the potential pitfalls when determining biomarker levels with different laboratory assays.

\section{The 'multi-marker approach' for assessing cardiovascular risk with biomarkers}

Large clinical trials are needed to complete phase three to five in the biomarker development process. Since atherogenesis and plaque disruption are multifactorial processes [4], it is highly unlikely that one single biomarker will be discovered for predicting cardiovascular events. Therefore, the current general opinion is shifting towards a multi-marker approach, in which a certain panel of biomarkers is assessed to determine an individual risk profile of a patient for cardiovascular disease [11]. Ideally, these biomarkers should not be correlated, as correlated biomarkers do not increase the predictive value. For example, by assuming an AUC (an objective manner to test the biomarker's ability to distinguish between diseased and healthy individuals) of 0.80 , the AUC increases to 0.88 if a second, weakly correlated biomarker was inserted into the analysis. In contrast, if a moderately correlated biomarker was inserted, the AUC only increases to 0.83 [46]. Another method of determining the additional value of multimarker versus single marker assessment is the calculation of the C-statistic (where ' $\mathrm{C}$ ' stands for concordance). The C-statistic is able to express the ability to discriminate better than the relative risk estimate [32, 47]. Higher values of the Cstatistics correspond with improved ability of discrimination.

However, the C-statistic cannot be used in our study design, as the Cstatistic can only be used to discriminate between two groups. Our serum marker study consisted of a total of four groups. Therefore, we performed a discriminant analysis of the biomarkers in order to evaluate which combination was able to discriminate between the study groups. In addition, we analyzed the correlation between patient characteristics and biomarkers by assessing their multicollinearity. There was no significant correlation between the biomarkers that were evaluated (chapter 3).

Nonetheless, the present consensus is that the multimarker approach is the way to go. For example, Varo et al. demonstrated that patients with elevated levels of both soluble CD40 ligand and troponin showed a marked increase 
in adjusted HR for adverse outcome (HR 4.3) when compared with soluble CD40 ligand alone (HR 1.9) [48]. However, the additional value of assessing multiple markers was expressed in this study as an increase in HR. As already mentioned, other statistical methods, such as the C-statistic, is preferred. Varo et al. failed to perform this kind of analysis. Ardigo et al. demonstrated that a combination of serum levels of multiple chemokines, such as CCL2, CCL7, CCL8, CCL13, and CXCL10, resulted in a high degree of accuracy in predicting clinically significant atherosclerotic heart disease in patients with and without clinically manifest coronary artery disease [49]. This observation is remarkable, since it is to be expected that the analyzed chemokines have a large correlation, which would result in only moderate improvement in the multimarker analysis. In contrast, Wang et al. measured 10 different biomarkers (CRP, B-type natriuretic peptide, $\mathrm{N}$-terminal proatrial natriuretic peptide, aldosterone, rennin, fibrinogen, D-dimer, plasminogen-activator inhibitor-1, homocysteine, and urinary albumin-tocreatinine ratio) in 3,209 participants of the Framingham Heart Study and monitored the participants for a follow-up period of 7.4 years [32]. Participants with high multimarker levels had a four times increased risk of death and a twofold increase in risk of major cardiovascular events when compared with participants with low multimarker levels. However, the use of multiple biomarkers added only moderately (as expressed by the C-statistic) to the predictive value of conventional cardiovascular risk factors (such as age, smoking, blood pressure, total cholesterol, and diabetes).

In the present thesis, a stepwise discriminant analysis of the markers MPO, leukocyte counts, CRP, fibrinogen, and PAPP-A was performed. It showed that MPO, PAPP-A (as determined with the ultrasensitive research kit) and leukocyte counts emerged as best markers to distinguish between different forms of coronary disease and healthy controls. These biomarkers might be promising biomarkers for predicting future cardiovascular events (chapter 3). Nevertheless, the combination of both leukocyte counts and MPO is expected to result in only moderate improvement in risk prediction as they are correlated. MPO is abundantly expressed by polymorphonuclear neutrophils and secreted during activation and thus is expected to be highly correlated with leukocytes [50]. Nonetheless, we did not find any significant correlation between MPO and leukocytes as expressed by their multicollinearity. Determining the predictive value of these biomarkers (as expressed as a relative risk estimate) was not possible with our current population size and follow-up. 
Future studies will need to analyze which combination of the available biomarkers is most promising, taken into account the characteristics of the 'ideal biomarker' as described above. In order to accurately described the additional value of this combined approach, statistical applications such as the AUC or C-statistic should be provided within the study. The panel of (preferably non-correlating) markers should then be used to assess the individual risk for developing cardiac events in the near future. According to the previously described roadmap for the identification of high-risk individuals, these people could be referred to specialized care and be analyzed further with non-invasive imaging methods, such as MRI.

\section{IMAGING OF ATHEROSCLEROSIS}

In the past years, research suggested that the vulnerability of an atherosclerotic plaque is determined by its composition rather than its degree of stenosis. Vulnerable plaques tend to have a large lipid rich necrotic core with a thin protective fibrous cap. In addition, intraplaque hemorrhage, plaque inflammation, and possibly also the density of the vasa vasorum are key characteristics of a vulnerable plaque [51-53]. According to the previously described roadmap high-risk individuals should be referred to specialized care facilities after primary screening (for example with the aid of biomarkers). Here, their atherosclerotic burden can be further analyzed with diagnostic imaging modalities. A number of these modalities have been tested for their ability to determine the composition of an atherosclerotic plaque in vivo $[54,55]$.

\section{Imaging atherosclerosis}

Many of these image modalities, like intravascular ultrasound, optical coherence tomography, angioscopy, and thermography, are invasive procedures and therefore less suitable as a screening modality for follow-up of patients. Non-invasive approaches are (surface) US, CT, PET-CT, SPECT, and MRI.

US is a rapid, low-cost, high resolution technique to assess plaque composition. Recent research reported on various sonographic markers, such as plaque echogenicity and irregularity, to identify high-risk patients [56]. Due to their large content of lipid rich necrotic core and/or hemorrhage, vulnerable plaques are thought to be more echolucent. The echogenicity of a plaque can be assessed visually or computer-aided with the GSM. Visual assessment of plaque echogenicity has poor reproducibility [57], whereas 
GSM proved to be more reliable and less operator dependent [58]. However, GSM assesses the median echogenicity of the entire plaque and does not demonstrate focal variations in echogenicity. Therefore, there is still no consensus on which cut off value for GSM discriminates best between vulnerable and stable atherosclerotic plaques. In addition, several studies have shown that plaque surface irregularity as assessed by B-mode US is a predictor of ischemic stroke $[59,60]$. However, more research is needed to see whether these initial findings can be reproduced. Nonetheless, the exact mechanisms between plaque surface irregularity and the occurrence of ipsilateral ischemic stroke is still unclear.

The main advantage of CT is its patient friendly use, its operator independency and finally, its relatively short image acquisition time, allowing for high-throughput screening of many patients. Technological improvements in CT scanning have been introduced, especially the use of multiple detectors that have an increased rotational speed. This drastically decreases the image acquisition time and limits the occurrence of motion artifacts [61]. Currently, the first 256-slice (or even higher) CT scanners and CT scanners with two x-ray tubes are being installed [62, 63]. Although it was demonstrated that CT can assess total plaque area, calcifications, and fibrous tissue, it was unable to accurately assess lipid rich necrotic cores in calcified plaques [64]. CT also does not have the excellent soft tissue contrast that can be acquired with MRI. Furthermore, it has the disadvantage of using ionizing radiation and usually requires additional administration of iodine contrast agents, which are potentially nephrotoxic $[65,66]$. The relatively large amount of ionizing radiation used in cardiac CT (approximately 8 to 10 $\mathrm{mSv}$, comparable to 4 to 5 years of normal background radiation dose in The Netherlands) is a serious drawback for its use in screening and followup of large populations. A recent paper by Brenner et al. emphasized this increase in radiation exposure of the general population due to CT applications and its negative consequences for future public health care [67]. In general, in applications that use ionizing radiation the patient's benefit should outweigh radiation exposure and the ALARA principle (As Low As Reasonably Achievable) should be applied.

PET-CT can be used to assess inflammation in plaques by measuring metabolic activity and glucose uptake (which is increased in inflammation) using ${ }^{18}$ Fluorodeoxyglucose (18 FDG). Tawakol et al. demonstrated in atherosclerotic rabbits that ${ }^{18} \mathrm{FDG}$ accumulates in macrophage-rich atherosclerotic plaques. In this way, vascular macrophage activity can be quantified non- 
invasively by using PET-CT [68]. Measurements in iliac, femoral, and carotid arteries of individuals with vascular disease proved highly reproducible [69]. Additional CT imaging enables co-localization of the 'hot spots' to anatomy. Therefore, PET-CT has similar disadvantages as CT. In addition, the prognostic value of atherosclerotic plaques imaging using ${ }^{18} \mathrm{FDG}$ uptake remains to be investigated [70].

SPECT imaging is performed by measuring the gamma ray emission of an administered radiopharmaceutical in multiple two-dimensional images with a gamma camera. With this dataset, a tomographic reconstruction can be constructed. In the past, atherosclerotic lesions of ApoE-/- mice were imaged with Tc ${ }^{99}$-Annexin- $\mathrm{V}$. Annexin- $\mathrm{V}$ is able to bind to phosphatidylserine, which is overexpressed in cell membranes of apoptotic cells and is additionally present in activated macrophages and stressed cells [71]. It was hypothesized that $\mathrm{Tc}^{99}$-labeled Annexin could identify apoptotic cells, activated macrophages and/or stressed cells in the lesions of symptomatic patients. Like PET, SPECT enables detection of very low concentrations of $\mathrm{Tc}^{99}$ Annexin- $\mathrm{V}$ in atherosclerotic plaques. However, due to its low resolution, SPECT needs additional CT for co-localization. Kietselaer et al. demonstrated human applicability of $\mathrm{Tc}^{99}$-Annexin-V SPECT imaging in a pilot study of four patients. These patients had a history of recent or remote transient ischemic attack and were scheduled for carotid endarterectomy. Prior to surgery, $\mathrm{Tc}^{99}$-Annexin-V SPECT imaging was performed, showing that in two patients who had suffered from TIA more than three months ago and who had since been treated with statins and antiplatelet therapy, no uptake of contrast agent was observed. In contrast, two patients who had suffered from TIA some days before imaging showed marked uptake of $\mathrm{Tc}^{99}$ Annexin-V in the culprit carotid artery [72]. These promising (proof-ofconcept) results showed that $\mathrm{Tc}^{99}$-Annexin- $\mathrm{V}$ imaging might have important consequences for patient stratification and treatment. However, larger studies and follow-up clinical trials have yet to be reported.

\section{Magnetic resonance imaging of (carotid) atherosclerotic plaques}

Over the past years, MRI has emerged as an accurate, non-invasive imaging modality which can assess carotid artery plaque components based on their biochemical properties. In addition, the contrast agents used are generally far less harmful than iodine contrast agents used in CT studies, even despite recent reports on the development of nephrogenic systemic fibrosis after administration of gadolinium-containing contrast agents [73, 74]. 
In 1996, Toussaint et al. were the first to demonstrate the assessment of plaque components of carotid artery plaques in vivo using MRI. It was suggested that by measuring signal intensities in $T_{2}$ weighted images, different plaque components like lipid core, fibrous tissue, calcification, and normal vessel wall tissue could be differentiated in a non-invasive manner [75].

In later publications by Yuan et al. and Shinnar et al. it was demonstrated that for accurate carotid plaque assessment, a high-resolution, multisequence approach had to be applied, using for instance $\mathrm{T}_{1^{-}}, \mathrm{T}_{2^{-}}$, proton density weighted (turbo) spin echo sequences, and TOF sequences [76, 77].

For optimal image quality, sequences need to be acquired with dedicated radiofrequency coils (which optimize SNR). To optimize contrast between lumen, vessel wall, and plaque, 'black blood' imaging sequences, such as DIR or MSDE sequences, are essential. DIR sequences utilize an inversion pulse to null the arterial blood signal [78]. Recently, Wang et al. showed an improved blood suppression in the carotid artery by using an MSDE sequence, resulting in a more accurate depiction of lumen boundaries and elimination of plaque-mimicking artifacts [79]. In addition, bright blood imaging techniques such as TOF sequences are applied to distinguish between areas of calcification and lumen boundaries, and can be applied to assess fibrous cap status (intact or ruptured) [80,81]. A $\mathrm{T}_{1}$ weighted TFE sequence can be used to identify areas of intraplaque hemorrhage [82, 83].

Despite the general opinion that the multi-sequence approach for carotid plaque imaging is essential, Cappendijk et al. recently demonstrated that the identification and quantification of lipid rich necrotic core areas could be accurately performed by the use of a single, $\mathrm{T}_{1}$ weighted TFE sequence instead of the multi-sequence approach [84]. This would enable more rapid scanning of a patient, increasing patient comfort and decreasing the risk of motion artifacts. With this approach, it was also demonstrated that patients with a carotid stenosis of greater than $70 \%$ and a recent transient ischemic attack or stroke displayed a larger lipid-rich necrotic core as assessed using the T1w TFE images compared to asymptomatic patients [85].

By administering commonly available gadolinium-based contrast agents Yuan et al. and Wasserman et al. demonstrated that differentiation between lipid core and fibrous cap could be improved [86, 87]. Contrast-enhanced MRI is essential for imaging neovascularisation in an atherosclerotic plaque. Kerwin et al. demonstrated that kinetic modeling of gadolinium-based con- 
trast agent wash-in and wash-out during dynamic MRI of carotid artery plaques enabled an assessment of neovascularization and the inflammatory burden of the plaque [88-90].

Macrophage-rich areas in atherosclerotic plaques can be visualized with the aid of USPIO's. These particles consist of a small iron oxide core (diameter 4 to $10 \mathrm{~nm}$ ) stabilized with a biodegradable coating to prevent particle aggregation and optimize pharmacokinetic behavior [91, 92]. Research demonstrated that USPIO with a long elimination half-life are most suitable for human atherosclerotic plaque imaging (e.g. ferumoxtran-10, Sinerem ${ }^{\circledR}$, Guerbet, France) [91]. $\mathrm{T}_{1}$ and $\mathrm{T}_{2} / \mathrm{T}_{2}{ }^{*}$ relaxation times are shortened by USPIO (as expressed by the $r_{1}$ and $r_{2}$ relaxivities of $100 \mathrm{mmol}^{-1} \mathrm{~s}^{-1}$ and 20 $\mathrm{mmol}^{-1} \mathrm{~s}^{-1}$ at $39^{\circ} \mathrm{C}$ and $0.47 \mathrm{~T}$, respectively). USPIO are taken up by macrophages, which modifies their magnetic properties. In general, $\mathrm{T}_{1}$ relaxivity is reduced, whereas $\mathrm{T}_{2} / \mathrm{T}_{2} *$ relaxivity is increased. Therefore, in USPIOenhanced MR imaging, it is preferred to use $T_{2} / T_{2}{ }^{*}$ weighted sequences (especially gradient echo sequences), in which the uptake of USPIO in postcontrast images is presented as signal loss. The uptake of USPIO in atherosclerotic plaque macrophages was demonstrated by Kooi et al., resulting in a decrease in post-contrast signal intensity in in vivo MRI of carotid arteries [93]. These findings were subsequently confirmed by Trivedi et al. [94, 95]. However, negative contrast enhancement associated with USPIO use is a drawback for its application in a clinical setting. In addition, both pre- and post-contrast imaging with an (optimal) 24 hour interval is required in these studies. New pulse sequences were developed to generate positive contrast enhancement. Alternatively, positive contrast enhancement can be generated by calculating the susceptibility gradient from the three-dimensional anatomical gradient echo images. The availability of both negative and positive contrast enhanced images might take away the need for pre-contrast imaging of these patients [91]. Nonetheless, in both negative and positive contrast-enhanced USPIO studies only a limited number of patients were evaluated. Larger future studies should focus on the predictive value of USPIO-enhanced MR plaque imaging for future ischemic events. In addition, future studies might investigate the relation between uptake of USPIO and drug treatment (e.g. statins).

Other contrast agents for plaque imaging are currently being developed, but their application is still limited to animals studies. Barkhausen et al. demonstrated the potential of gadofluorine in imaging of atherosclerosis in rabbits [96]. Sirol et al. confirmed these initial findings and additionally demon- 
strated a positive correlation between signal enhancement and lipid rich necrotic core areas [97]. Recently, Yasuda et al. demonstrated in ApoE-/mice that Evans-Blue Gd-DTPA is a useful contrast agent for atherosclerotic plaque imaging. Evans-Blue dye interacts with the extracellular matrix and vascular SMC when vascular endothelial cells are injured. Therefore, the Evans-Blue Gd-DTPA complex was able to accumulate at atherosclerotic lesions, which are characterized by endothelial injury and dysfunction [98].

The main disadvantages of the contrast agents mentioned above are the lack of FDA approval and thus clinical availability (especially gadofluorine and USPIO), the relatively poor relaxivity (with the exception of USPIO), their ability to accumulate within the body (in the case of USPIO), and their rapid excretion out of the human body (Gd-DTPA). Therefore, new contrast agents are being designed to bind to disease specific molecules ('molecular imaging') and are currently translated to human studies.

Another limitation is that the studies in which optimal scan settings for plaque imaging are evaluated have been rather small. Until now, no large clinical trials using MR plaque imaging to determine patient stratification have been reported. Several large trials are currently ongoing, such as the High Risk Plaque Initiative and the Multi-Ethnic Study of Atherosclerosis [54]. Saam et al. performed bilateral MR imaging of the carotid in 192 patients and demonstrated that complex lesions (American Heart Association type VI with luminal surface defects, hemorrhage and/or calcified nodules) were present in a substantial number of patients with a stenosis $\leq 50 \%$, but the prognostic value of plaque composition (as assessed with MRI) for cerebrovascular events was not analyzed [99]. Therefore, many questions on the clinical application of MR plaque imaging to determine carotid artery plaque composition still need answering. Future studies could focus on the ability of MR plaque imaging to predict cardiovascular complications based on plaque features. Also, studies might investigate whether MR plaque imaging is a better modality for stratifying patients scheduled for CEA, when compared with conventional screening methods such as duplex ultrasound. For example, Altaf et al. recently demonstrated that intraplaque hemorrhage (as assessed by MR plaque imaging of symptomatic patients with mild to moderate $(30-69 \%)$ carotid stenosis) was a good predictor of ipsilateral stroke or TIA $[100,101]$.

It is essential that (parallel to optimizing hardware and scanning protocols) larger clinical trials are undertaken to assess the true power of MRI of athe- 
rosclerosis in a clinical setting. The next step is to evaluate the value of MRI for coronary artery plaque composition.

\section{Contrast-enhanced magnetic resonance imaging of carotid artery atherosclerosis with the albumin-binding blood pool agent gadofosveset}

Gadofosveset (MS-325, Vasovist ${ }^{\circledR}$, Bayer Schering Pharma, Germany) was the first blood pool agent to become commercially available. It consists of a diphenylcyclohexyl group attached to a gadolinium chelate by a phosphodiester linkage, enabling it to bind reversibly to albumin. After administration, a large fraction is immediately bound to albumin, whereas a small fraction remains unbound. By binding to albumin the elimination half-life increases dramatically and $\mathrm{r}_{1}$ relaxivity is increased approximately ten times compared with other gadolinium complexes [102, 103]. Its main clinical advantages are higher post-contrast signal intensities at lower doses when compared with other, more commonly used Gd-chelates. Currently, gadofosveset is mainly used in MR angiography studies [104]. To the best of our knowledge, we were amongst the first to investigate the potential use of gadofosveset for improvement of atherosclerotic plaque imaging.

Albumin is the most abundant protein present in the circulation [105]. It leaves the circulation only through the fenestrated endothelium of capillaries and the dysfunctional endothelium of atherosclerotic arteries. Analysis of the distribution of albumin across the normal human aortic wall showed low to moderate amounts of albumin in the intima, with lower amounts in the media and higher amounts in the adventitia. Very large amounts were observed surrounding the vasa vasorum. In aortas with intimal thickening, the relative distribution remained unaltered, but in general, higher amounts of albumin were observed than in healthy aortic walls [106, 107]. A positive correlation between plaque size, micro-vessel density, and plaque albumin content was demonstrated by Zhang et al. [108]. Based on these observations, we hypothesized that gadofosveset might have added value for contrast-enhanced MR imaging of (carotid) atherosclerotic lesions. First, this hypothesis was evaluated in an atherosclerotic rabbit model, where a significant difference in signal enhancement was shown between atherosclerotic and normal rabbit aortas. In contrast, Gd-DTPA showed signal enhancement in both atherosclerotic and normal rabbit aortas and was therefore unable to distinguish between diseased and healthy vessel walls (chapter 4). In a study in humans, we showed that gadofosveset administration resulted in a significantly higher signal enhancement in atherosclerotic plaques of symptomatic patients, whereas no apparent signal enhancement was ob- 
served in plaques of asymptomatic patients with carotid stenosis (chapter 5). This suggests that gadofosveset might be used to distinguish between the carotid lesions of symptomatic and asymptomatic patients.

Recently, Cornily et al. used another albumin-binding contrast agent (B22956/1) in MR plaque imaging of New Zealand White rabbits [109]. In this study, atherosclerotic $(n=7)$ and control $(n=3)$ rabbits both received Gd-DTPA and B22956/1 with a one week interval. MR acquisition was performed up to 2 hours after contrast administration. Similar observations in the B22956/1 group were made as in our gadofosveset animal experiments: a significantly higher signal enhancement was measured in atherosclerotic rabbits receiving B22956/1 when compared with controls (39.8\% versus $9.5 \%, p<0.0001)$. Cornily et al. also demonstrated that early plaque enhancement after administration of B22956/1 correlated moderately with neovessel density within the plaque $(\mathrm{r}=0.42, p<0.01)$ and that signal increase after 2 hours correlated well with macrophage density as expressed by positive CD68 immunohistochemical staining ( $\mathrm{r}=0.71, p<0.01)$. However, in contrast to our study, no correction for the much higher $\mathrm{r}_{1}$ relaxivity of B22956/1 in comparison with Gd-DTPA was made $\left(27 \mathrm{mM}^{-1} \mathrm{~s}^{-1}\right.$ and 3.6 $\mathrm{mM}^{-1} \mathrm{~s}^{-1}$ in these study conditions, respectively) and identical post-contrast imaging intervals were used for both contrast agents, despite their differences in elimination half-life. This could partly explain the higher signal enhancement observed after administration of B22956/1. In addition, B22956/1 is still an experimental contrast agent, limiting its use in human applications (in contrast to gadofosveset used in our studies, which is FDA approved for human applications in MR angiography and which might be used 'off label' for atherosclerotic plaque imaging).

Although initial results using gadofosveset are promising, our current studies consisted of an animal study and one human feasibility study with a small sample size. Although several mechanisms were suggested, we have not yet been able to determine the exact mechanism of gadofosveset uptake in atherosclerotic plaques. In addition, no comparison of the potential of contrast-enhanced plaque imaging in human applications was made between gadofosveset and Gd-DTPA. Additional studies are warranted to validate and reproduce these initial findings in large studies. More knowledge on the optimal gadofosveset dose and post-contrast imaging delay to be used in imaging protocols needs to be obtained. Future studies should investigate the association between post-contrast signal enhancement of gadofosveset in carotid plaques and the occurrence of near-future neurologic events. Al- 
so, the potential use of gadofosveset in atherosclerotic imaging of other vascular beds, such as coronaries, needs to be investigated. Since gadofosveset is currently used in MR angiography studies, delayed post-contrast imaging might be used additionally to analyze culprit stenoses in more detail. Finally, besides the advantages of gadofosveset over Gd-DTPA already mentioned (i.e. increased relaxivity, increased circulating half-life), we demonstrated in this thesis that gadofosveset is able to differentiate between atherosclerotic and normal vessel walls, whereas Gd-DTPA increased signal intensity of all arterial vessel walls. These initial findings warrant future comparison of the potential of gadofosveset and Gd-DTPA in atherosclerotic plaque imaging.

\section{Future developments in atherosclerotic plaque imaging with MRI}

Future developments in atherosclerotic plaque imaging will include developments in MRI field strength, dedicated radiofrequency coils, and contrast agents.

In this thesis we used a small, $47 \mathrm{~mm}$ diameter microscopy coil (Philips Healthcare, Best, The Netherlands) and a $\mathrm{T}_{1}$-weighted, 2D DIR spin echo sequence for carotid plaque imaging. It was placed directly on the carotid bifurcation in order to obtain a high SNR, and thus better image quality. Recently, commercial multichannel phased array surface coils that have a high SNR over a long distance in the longitudinal direction, have become available for carotid plaque imaging. In addition, these coils are able to analyze both carotids simultaneously, enabling direct comparison of both carotids in one session [110]. In the case of symptomatic carotid artery atherosclerosis, this could lead to more information about the differences in plaque composition between the carotid lesions. Future developments of these radiofrequency coils will result in better SNR (especially in a larger area of interest), leading to better image quality and/or faster imaging protocols.

Nowadays, many hospitals are changing to clinical MRI scanners with higher field strengths. SNR is roughly linearly related to field strength, thus an increase in field strength usually results in an increase in SNR. This can be used to acquire images with a higher spatial resolution, and thus with less partial volume effects, or, alternatively, it can be used to reduce scan time and thus the occurrence of motion artifacts. However, there is a trade-off. First, tissues differ in their magnetic susceptibility and there is an increase in susceptibility artifacts at higher field strengths. Second, the energy that is 
deposited into the patient's body is increased at higher field strengths. As a result, the limit of the SAR is reached faster. Imaging protocols will need to be redesigned to meet $S A R$ limitations. Finally, $T_{1}$ values of different tissues at higher field strength are longer and differences in $T_{1}$ values become smaller, making it possibly more difficult to differentiate between various kinds of tissue. With respect to the future use of higher field strengths in MRI, protocols for atherosclerotic plaque imaging need to be redesigned with these specific (dis)advantages of higher field strengths in mind. Recent publications have already demonstrated the increased SNR and CNR of plaque imaging on 3.0 Tesla systems compared with 1.5 Tesla scanners, resulting in higher quality images at higher resolutions [111-115].

\section{MOLECULAR IMAGING OF ATHEROSCLEROSIS}

Targeted contrast agents could aid in increasing our knowledge of pathogenesis of diseases and might also enable target-delivered drug therapy. Imaging with the use of specific targeted contrast agents is called molecular imaging. In a joint workshop of experts from the Society of Nuclear Medicine and the Radiological Society of North America in April 2005 molecular imaging was defined as follows: 'Molecular imaging techniques directly or indirectly monitor and record the spatio-temporal distribution of molecular or cellular processes for biochemical, biological, diagnostic, or therapeutic applications' [116].

\section{The building blocks of a molecular contrast agent}

Typically, a molecular imaging contrast agent consists of three essential parts. The first part consists of a (preferably disease specific) ligand. Second, this ligand is bound to a certain carrier, enabling the contrast agent to be transported throughout the human body without causing immunological or toxic reactions. Third, the ligand-carrier-complex needs to be attached to labels that can be detected by the image modality of interest. In the case of MRI, these are usually gadolinium or iron oxide particles [117].

Molecular imaging ligands need to be selected carefully. Preferably, they should have a high specificity for the disease of interest. The number of available binding sites should be as large as possible, in order to obtain a high local concentration of bound contrast agent (and thus, high signal intensity). Ideally, the target should be easily accessible for the contrast agent. Finally, the binding affinity to the target to the ligand should be high, in 
order to prevent non-specific binding of contrast agent to the target molecule.

In the case of molecular MR imaging, several carriers have been proposed for applications in molecular imaging. Since MR imaging requires relatively large local concentrations of gadolinium to acquire measurable signal enhancements after contrast administration, many of these carriers are manufactured in a way that they amplify MR signal intensity. Nowadays, the most extensively validated carriers used are perfluorocarbon, lipid based nanoparticles, and iron oxide particles.

Perfluorocarbon nanoparticles are carriers that are capable delivering 50,000 or more gadolinium molecules to a certain target [118]. These nanoparticles can increase relaxivity with a factor of 100,000 or more when compared with clinically used paramagnetic contrast agents [118]. In addition, their core can be filled with drugs, enabling target-delivered drug treatment [119].

Lipid-based nanoparticles, such as liposomes and micelles, consist of a monolayer or bilayer membrane of fatty acids with an aqueous core. Gadolinium-chelates can be incorporated into this complex to increase its relaxivity [120]. Potential targets for imaging can be incorporated into the membrane $[121,122]$. However, a potential drawback for liposomes is their limited penetration in tissue.

Iron oxide particles are commercially available in two diameters (although various sizes are possible): $60-150 \mathrm{~nm}$ (small particles of iron oxide ) and 15$30 \mathrm{~nm}$ (USPIO). These particles result in decrease of signal on $\mathrm{T}_{2}{ }^{*}$ weighted images due to their $\mathrm{T}_{2}{ }^{*}$ shortening effects. The advantages and disadvantages of iron oxide particles were already discussed in a previous paragraph.

A new method of increasing signal amplification for molecular imaging purposes is applying a wedge to which multiple gadolinium molecules can be attached. By using this approach, relaxivity of a contrast agent can be dramatically increased, because the total load of gadolinium molecules is increased [123].

\section{Molecular imaging of atherosclerosis}

With respect to this thesis and cardiovascular imaging, multiple targets for molecular imaging have been proposed, such as adhesion molecules $(\mathrm{V}$ - 
CAM, E-selectin), protease activity (MMP), lipids (oxLDL), and the macrophage scavenger receptor $[66,122]$.

In a study by Winter et al., molecular MR imaging of the alpha-v-beta-3integrin (a marker for angiogenesis) attached to paramagnetic nanoparticles was demonstrated on atherosclerotic New Zealand White rabbits [124]. Winter et al. also used molecular MR imaging to show inhibition of angiogenesis in these cholesterol-fed rabbit aortas when treated with alpha-vbeta-3-integrin targeted nanoparticles filled with fumagillin (an angiogenesis inhibiting drug) [125]. By using paramagnetic liposomes targeted to alpha-vbeta-3-integrin, Mulder et al. were able to quantitate the presence of tumor angiogenesis in a melanoma mouse tumor model by using MRI. In addition, the effect of two administered angiogenesis-inhibiting drugs (anginex and endostatin) could be assessed with this approach [126].

The potential of molecular imaging of fibrin in thrombi was demonstrated by Flacke et al., who used an anti-fibrin monoclonal antibody attached to paramagnetic nanoparticles to visualize thrombi with MRI. After cloth formation in the jugular vein of dogs, the thrombi were entrapped between snare closures and the contrast agent was allowed to locally incubate for one hour. After contrast incubation, the thrombi were reexposed to the general circulation and imaged with MRI, showing significant enhancement of the cloth after exposure to the fibrin-targeted nanoparticle when compared with control (non-targeted) nanoparticles [127]. Molecular imaging of fibrin was also demonstrated by Botnar et al., who used a fibrin-binding, gadoliniumcontaining peptide (EP-1873) to image thrombi that were pharmaceutically induced in atherosclerotic rabbits [128]. In subsequent studies, Spuentrup et al. have made impressive progress in molecular imaging of fibrin, showing increased signal intensity on blood cloths after administration of EP-2104R (which is similar to EP-1873, but which is specific for buman fibrin) in animals models of atherosclerosis, deep venous thrombosis and pulmonary embolism. In these animal models, human thrombi were introduced in the animals (mostly swine), after which contrast agent was administered [128130]. Recently, the initial results of human application of EP-2104R were published. This study showed that on enhanced images, thrombi demonstrated high signal enhancement, typically on its surface. In eleven patients with thrombi in the left ventricle, left or right atrium, carotid artery, or thoracic aorta it was demonstrated that the thrombus could be delineated well from surrounding blood, as expressed by the significantly increased contrast-to-noise ratio for both thrombus versus blood and thrombus versus 
soft tissue. Therefore, EP-2104R allows for in vivo molecular MR imaging of thrombi [131].

Recently, Lancelot et al. demonstrated the possibility of molecular imaging of matrix metalloproteinases by using the contrast agent P947 ex vivo on atherosclerotic rabbit plaques and in vivo in ApoE-/- mice [132].

\section{Novel targets for molecular imaging of atherosclerosis}

Multiple studies proposed novel potential targets for molecular imaging. Kelly et al. identified 30 peptides of which some had homology to known plaque proteins by analyzing early atherosclerotic lesions in ApoE-/- mice using in vivo phage display. Amongst these were leukemia inhibitory factor and transferrin, which were upregulated in early stages of murine atherosclerosis, and VLA-4, which is able to bind to VCAM-1 expressed on endothelial cells. These peptides might be potential targets for molecular imaging of atherogenesis, but it still needs to be investigated if these animal study results can be substantiated in patients [133]. Megens et al. evaluated the use of CNA35 as a marker for collagen in both ex vivo and in vivo tissue samples of both ApoE-/- and control mice. They demonstrated that strong CNA35 labeling was found in murine plaques, making CNA35 a potential target for molecular imaging of collagen within atherosclerotic plaques. CNA35 imaging in mice organs revealed that the endothelium might be a limiting factor in in vivo uptake [134]. In elastic arteries, the CNA35 probe was unable to enter the vessel wall. In addition, CNA35 was present in organs with fenestrated or discontinuous endothelium (such as spleen, kidney, and liver), whereas no CNA35 was present in organs with continuous endothelium. For cardiovascular imaging purposes, this 'limitation' has important consequences. In healthy arteries, the endothelium is intact and forms a continuous inner lining of the vascular lumen, making it impossible for this contrast agent to penetrate into the vessel wall. However, in atherosclerotic plaques this endothelium is damaged and more permeable [135], enabling the possible in vivo uptake of this contrast agent into plaques. Hong et al. synthesized a novel (fluorescein- or radio-isotope) peptide able to home onto IL-4 receptor on murine atherosclerotic plaques and thus demonstrated its potential as a future target for molecular imaging [136]. In order to become clinically applicable however, this peptide needs to be attached with another imaging label, since fluorescent techniques are difficult to apply in human studies. As already mentioned, Cleutjens et al. were able to identify two (as yet unidentified) antibody responses to cDNA products. Antibodies were subsequently evaluated on serum samples of a cohort of patients with pe- 
ripheral atherosclerotic lesions, different stages of coronary artery disease, and healthy controls [40]. The great sensitivity and specificity of the antibodies promise great potential for molecular imaging applications. However, as yet it is not known whether the antigens for these antibodies are located within the plaque itself. If they are, these antigens might be potential targets for a molecular imaging contrast agent with the antibodies as ligands.

Phage display could aid in the rapid development of potential ligands for molecular imaging, as demonstrated by Kelly et al. and Cleutjens et al. in the previous paragraph [40,133]. For example, with this approach Hong et al. identified a protein for the interleukin- 4 receptor expressed on atherosclerotic plaques and suggested it as a potential target for molecular imaging applications [133].

\section{Most promising molecular imaging applications in atherosclerosis}

Molecular imaging, especially in cardiovascular disease, is still in its formative years and it is difficult to predict which technological improvements will be developed over the next decades. Nonetheless, it is obvious that molecular imaging has great potential for future clinical and research applications. Molecular imaging has the power to develop specific target-delivered drug therapy, as demonstrated by Winter et al. and Tran et al. [125, 137]. Furthermore, by imaging specific molecular targets and follow these targets in time and/or space, our knowledge of molecular processes of a disease in vivo can increase. Molecular imaging could also aid in the (more rapid and efficient) development of drugs and therapeutic strategies, for molecular imaging enables the use of fewer animals on which a new drug is tested and additionally, drug effect on the disease of interest can be assessed more efficiently. In our opinion however, nanoparticles, liposomes and fibrintargeted contrast agents have the greatest potential for clinical applications. The liposome particles used by Mulder et al. and the nanoparticles used by Winter et al. could also be used as drug-carriers, enabling specific targetdelivered drug therapy next to the imaging opportunities already demonstrated. This would allow the delivery of high concentrations of drugs to a site of interest and reduce the number of side effects associated with the systemic administration of these drugs. The potential of the EP-1873 contrast agent was already proven by publications by Spuentrup et al., who further developed the EP-1873 probe for use in human applications (under the name EP-2104R). As already discussed, the initial results of the application of this contrast agent have recently been published and the initiation of subsequent phase II and III trials is expected. 


\section{Potential pitfalls in molecular imaging of atherosclerosis}

Many of the molecular imaging studies published consist of 'proof-ofconcept' or 'feasibility' studies. In addition, the costs of preparing these kinds of custom made contrast agents are high and experiments are usually labor-intensive. For this reason, the number of observations in these studies is usually scant and results should be interpreted with these limitations in mind. In order to confirm the initial findings and to lift molecular imaging to a clinical level, the experiments need to be reproduced in larger study groups.

Since MRI has relatively low sensitivity, it remains to be seen if concentrations of target molecules are high enough to detect contrast after administration. In line with this insensitivity, it remains to be seen if the amplification techniques developed (such as the liposomes and nanoparticles discussed earlier) are adequate enough to overcome these thresholds. Also, a large number of studies performed with molecular imaging are often feasibility studies in small numbers of animals. This is largely caused by the high costs associated with contrast agent design and production. With this respect, we recommend that with regard to possible clinical applications, ligands for molecular imaging should preferably consist of peptides instead of antibodies, because of the high costs and sometimes limited availability (if the peptide or antibody is not commercially available and has to be custommade) of the latter. In addition, the larger size of antibodies in comparison to peptides might additionally limited their use as molecular imaging contrast agent due to their limited penetrative ability. However, if molecular imaging applications are limited to in vitro and small animal studies, antibodies might be suitable targets, since their binding affinity and specificity are usually excellent.

The use of novel contrast agents for human applications is associated with new challenging issues, such as pharmacokinetics and toxicity. These will have to be investigated and are potential obstacles for several of the currently known contrast agents. Nonetheless, it is expected that the progression of molecular imaging studies will continue. Multiple research groups from the pharmaceutical industry have already installed dedicated imaging laboratories to allow high-through put validation of potential candidates for molecular imaging [138]. In addition, large corporate research programmes, in which molecular imaging was incorporated, were initiated, such as the Center for Translational and Molecular Medicine, which is 'dedicated to the development of medical technologies that enable the design of new and 
personalized treatments for the main causes of mortality and diminished quality of life, and the rapid translation of these treatments to the patient' [139]. This proves that the faith in the potential of molecular imaging applications is enormous.

\section{CONCLUSION AND OUTLOOK}

Atherosclerosis is one of the leading causes of death in industrialized countries [140]. In order to reduce atherosclerosis-related mortality and morbidity, and health care costs associated herewith, we need to shift from a curative to a more preventive treatment strategy.

With this respect, a roadmap was suggested to identify the patient at risk for near-future cardiovascular complications such as myocardial infarction or stroke [8-10]. In this thesis, two aspects of this roadmap were evaluated: the use of biomarkers for cardiovascular disease and MRI imaging of atherosclerotic lesions. Sophisticated techologies, such as genomics and proteomics, will have the potential to lead to an increase in the number of biomarkers for cardiovascular disease. However, many studies on biomarkers for cardiovascular disease are limited to phase three of the biomarker development process, in which retrospective studies assess the ability of a biomarkers to distinguish between diseased and healthy individuals. Further development of biomarkers require large prospective studies and costeffectiveness studies, which are costly and labor-intensive. However, such studies are vital for clinical validation of biomarkers for cardiovascular disease. In addition, it is unlikely that one single biomarker for cardiovascular disease will be used in a clinical setting. In this thesis we demonstrated that the predictive value of current well-validated biomarkers (such as CRP and fibrinogen) is moderate and comparable with other, less-validated biomarkers (such as sCD40L, IL-6, and oxLDL). In addition, we demonstrated the ability of the new biomarkers MPO and PAPP-A to distinguish between cardiovascular patients and healthy controls. However, due to our limited population size and follow-up period, we were not able to assess the predictive value of myeloperoxidase for future cardiovascular complications.

This thesis further investigated the potential of non-invasive MRI for atherosclerotic plaque imaging, especially with the aid of a novel albuminbinding blood pool agent, gadofosveset. We showed that gadofosveset has a large potential for future clinical purposes, since initial results showed that it was able to distinguish between atherosclerotic and normal vessel wall of 
New Zealand White rabbits, and between atherosclerotic lesions of symptomatic and asymptomatic patients. Because gadofosveset is FDA approved and has recently become commercially available, we expect that plaque imaging applications of gadofosveset will have high potential. Although the progression made in MR imaging of atherosclerosis in the past twelve years is spectacular, almost all studies, including our own two studies within this thesis, focus on optimalization of technological hardware, sequences protocols, and contrast agent applications. The time is right for MR plaque imaging to take the next step, as for it to become clinically applicable, large (multicenter) trials focusing on the quantification of the predictive value for cardiovascular events of plaque components should be undertaken.

With respect to molecular imaging, near-future clinical applications are not to be expected. Molecular imaging applications are still under investigation and in the near future, its application will primarily be in an experimental setting. However, it is to be expected that selected targeted contrast agents will find their way to the clinic in the coming decades. 


\section{REFERENCES}

1. Ross R. Atherosclerosis: an inflammatory disease. N Engl J Med. 1999; 340: 115-26.

2. Lusis AJ. Atherosclerosis. Nature. 2000; 407: 233-41.

3. Willeit J, Kiechl S. Biology of arterial atheroma. Cerebrovasc Dis. 2000; Suppl 5:1-8.

4. Hansson GK. Inflammation, atherosclerosis, and coronary artery disease. N Engl J Med. 2005; 1685-95.

5. Anderson KM, Odell PM, Wilson PW, Kannel WB. Cardiovascular disease risk profiles. Am Heart J. 1991; 121: 293-8.

6. Manhem K, Dotevall A, Wilhelmsen L, Rosengren A. Social gradients in cardiovascular risk factors and symptoms of Swedish men and women: the Goteborg MONICA Study 1995. J Cardiovasc Risk. 2000; 7:359-68.

7. Emmerich J, Ridker PM. Can fishing for new genes catch patients at risk for coronary artery disease? Clin Chem. 2008; 54: 453-5.

8. Fuster V. Epidemic of cardiovascular disease and stroke: the three main challenges. Presented at the 71th scientific sessions of the American Heart Association. Dallas, Texas. Circulation. 1999; 99: 1132-7.

9. Naghavi M, Libby P, Falk E, Casscells SW, Litovsky S, Rumberger J, Badimon JJ, Stefanadis C, Moreno P, Pasterkamp G, Fayad Z, Stone PH, Waxman S, Raggi P, Madjid M, Zarrabi A, Burke A, Yuan C, Fitzgerald PJ, Siscovick DS, de Korte CL, Aikawa M, Juhani Airaksinen KE, Assmann G, Becker CR, Chesebro JH, Farb A, Galis ZS, Jackson C, Jang IK, Koenig W, Lodder RA, March K, Demirovic J, Navab M, Priori SG, Rekhter MD, Bahr R, Grundy SM, Mehran R, Colombo A, Boerwinkle E, Ballantyne C, Insull W Jr, Schwartz RS, Vogel R, Serruys PW, Hansson GK, Faxon DP, Kaul S, Drexler H, Greenland P, Muller JE, Virmani R, Ridker PM, Zipes DP, Shah PK, Willerson JT. From vulnerable plaque to vulnerable patient: a call for new definitions and risk assessment strategies, part I. Circulation. 2003; 108: 1664-72.

10. Naghavi M, Libby P, Falk E, Casscells SW, Litovsky S, Rumberger J, Badimon JJ, Stefanadis C, Moreno P, Pasterkamp G, Fayad Z, Stone PH, Waxman S, Raggi P, Madjid M, Zarrabi A, Burke A, Yuan C, Fitzgerald PJ, Siscovick DS, de Korte CL, Aikawa M, Airaksinen KE, Assmann G, Becker CR, Chesebro JH, Farb A, Galis ZS, Jackson C, Jang IK, Koenig W, Lodder RA, March K, Demirovic J, Navab M, Priori SG, Rekhter MD, Bahr R, Grundy SM, Mehran R, Colombo A, Boerwinkle E, Ballantyne C, Insull W Jr, Schwartz RS, Vogel R, Serruys PW, Hansson GK, Faxon DP, Kaul S, Drexler H, Greenland P, Muller JE, Virmani R, Ridker PM, Zipes DP, Shah PK, Willerson JT. From vulnerable plaque to vulnerable patient: a call for new definitions and risk assessment strategies, part II. Circulation. 2003; 108: 1772-8.

11. Koenig W, Khuseyinova N. Biomarkers of atherosclerotic plaque instability and rupture. Arterioscler Thromb Vasc Biol. 2007; 27: 15-26.

12. Khuseyinova N. Koenig W. Biomarkers of outcome from cardiovascular disease. Curr Opin Crit Care. 2006; 12: 412-9.

13. Gerszten RE, Wang TJ. The search for new cardiovascular biomarkers. Nature. 2008; 451: 949-52.

14. Pepe MS, Etzioni R, Feng Z, Potter JD, Thompson ML, Thornquist M, Winget M, Yasui Y. Phases of biomarker development for early detection of cancer. J Natl Cancer Inst. 2001; 93: 1054-61. 
15. Ridker PM, Buring JE, Shih J, Matias M, Hennekens CH. Prospective study of Creactive protein and the risk of future cardiovascular events among apparently healthy women. Circulation. 1998; 98: 731-3.

16. Tracy RP, Lemaitre RN, Psaty BM, Ives DG, Evans RW, Cushman M, Meilahn EN, Kuller LH. Relationship of C-reactive protein to risk of cardiovascular disease in the elderly. Results from the Cardiovascular Health Study and the Rural Health Promotion Project. Arterioscler Thromb Vasc Biol. 1997; 17: 1121-7.

17. Morrow DA, Rifai N, Antman EM, Weiner DL, McCabe CH, Cannon CP, Braunwald E. C-reactive protein is a potent predictor of mortality independently of and in combination with troponin $\mathrm{T}$ in acute coronary syndromes: a TIMI 11A substudy. Thrombolysis in Myocardial Infarction. J Am Coll Cardiol. 1998; 31: 1460-5.

18. Arima H, Kubo M, Yonemoto K, Doi Y, Ninomiya T, Tanizaki Y, Hata J, Matsumura K, Iida M, Kiyohara Y. High-sensitivity C-reactive protein and coronary heart disease in a general population of Japanese: the Hisayama study. Arterioscler Thromb Vasc Biol. 2008; 28: 1385-91.

19. Ridker PM, Buring JE, Cook NR, Rifai N. C-reactive protein, the metabolic syndrome, and risk of incident cardiovascular events: an 8-year follow-up of 14719 initially healthy American women. Circulation. 2003; 107: 391-7.

20. Ernst E. Fibrinogen as a cardiovascular risk factor--interrelationship with infections and inflammation. Eur Heart J. 1993; 14 Suppl K: 82-7.

21. Maresca G, Di Blasio A, Marchioli R, Di Minno G. Measuring plasma fibrinogen to predict stroke and myocardial infarction: an update. Arterioscler Thromb Vasc Biol. 1999; 19: 1386-77.

22. Stec JJ, Silbershatz H, Tofler GH, Matheney TH, Sutherland P, Lipinska I, Massaro JM, Wilson PF, Muller JE, D'Agostino RB Sr. Association of fibrinogen with cardiovascular risk factors and cardiovascular disease in the Framingham Offspring Population. Circulation. 2000; 102: 1634-8.

23. Baker IA, Pickering J, Elwood PC, Bayer A, Ebrahim S. Fibrinogen, viscosity and white blood cell count predict myocardial, but not cerebral infarction: evidence from the Caerphilly and Speedwell cohort. Thromb Haemost. 2002; 87: 421-5.

24. Luo Y, Jiang D, Wen D, Yang J, Li L. Changes in serum interleukin-6 and highsensitivity C-reactive protein levels in patients with acute coronary syndrome and their responses to simvastatin. Heart Vessels. 2004; 19: 257-62.

25. Fibrinogen Studies Collaboration, Kaptoge S, White IR, Thompson SG, Wood AM, Lewington S, Lowe GD, Danesh J. Associations of plasma fibrinogen levels with established cardiovascular disease risk factors, inflammatory markers, and other characteristics: individual participant meta-analysis of 154,211 adults in 31 prospective studies: the fibrinogen studies collaboration. Am J Epidemiol. 2007; 867-79.

26. Mach F, Schönbeck U, Sukhova GK, Bourcier T, Bonnefoy JY, Pober JS, Libby P. Functional CD40 ligand is expressed on human vascular endothelial cells, smooth muscle cells, and macrophages: implications for CD40-CD40 ligand signaling in atherosclerosis. Proc Natl Acad Sci USA. 1997; 94: 1931-6.

27. Lutgens E, Cleutjens KB, Heeneman S, Koteliansky VE, Burkly LC, Daemen MJ. Both early and delayed anti-CD40L antibody treatment induces a stable plaque phenotype. Proc Natl Acad Sci USA. 2000; 97: 7464-9.

28. Biasucci LM, Liuzzo G, Fantuzzi G, Caligiuri G, Rebuzzi AG, Ginnetti F, Dinarello CA, Maseri A. Increasing levels of interleukin (IL)-1Ra and IL-6 during the first 2 days of hospitalization in unstable angina are associated with increased risk of in-hospital coronary events. Circulation. 1999; 99: 2079-84. 
29. Maier W, Altwegg LA, Corti R, Gay S, Hersberger M, Maly FE, Sütsch G, Roffi M, Neidhart M, Eberli FR, Tanner FC, Gobbi S, von Eckardstein A, Lüscher TF. Inflammatory markers at the site of ruptured plaque in acute myocardial infarction: locally increased interleukin- 6 and serum amyloid A but decreased C-reactive protein. Circulation. 2005; 111: 1355-61.

30. Ehara S, Ueda M, Naruko T, Haze K, Itoh A, Otsuka M, Komatsu R, Matsuo T, Itabe H, Takano T, Tsukamoto Y, Yoshiyama M, Takeuchi K, Yoshikawa J, Becker AE. Elevated levels of oxidized low density lipoprotein show a positive relationship with the severity of acute coronary syndromes. Circulation. 2001; 103: 1955-60.

31. Toshima S, Hasegawa A, Kurabayashi M, Itabe H, Takano T, Sugano J, Shimamura K, Kimura J, Michishita I, Suzuki T, Nagai R. Circulating oxidized low density lipoprotein levels. A biochemical risk marker for coronary heart disease. Arterioscler Thromb Vasc Biol. 2000; 20: 2243-7.

32. Wang TJ, Gona P, Larson MG, Tofler GH, Levy D, Newton-Cheh C, Jacques PF, Rifai N, Selhub J, Robins SJ, Benjamin EJ, D'Agostino RB, Vasan RS. Multiple biomarkers for prediction of first major cardiovascular events and death. N Engl J Med. 2006; 355 : 2631-9.

33. Tabibiazar R, Wagner RA, Deng A, Tsao PS, Quertermous T. Proteomic profiles of serum inflammatory markers accurately predict atherosclerosis in mice. Physiol Genomics. 2006; 25: 194-202.

34. Choi KM, Lee JS, Kim EJ, Baik SH, Seo HS, Choi DS, Oh DJ, Park CG. Implication of lipocalin-2 and visfatin levels in patients with coronary heart disease. Eur J Endocrinol. 2008; 158: 203-7.

35. Shlipak MG, Ix JH, Bibbins-Domingo K, Lin F, Whooley MA. Biomarkers to predict recurrent cardiovascular disease: the Heart and Soul Study. Am J Med. 2008; 121: 50-7.

36. Kraaijeveld AO, de Jager SC, de Jager WJ, Prakken BJ, McColl SR, Haspels I, Putter H, van Berkel TJ, Nagelkerken L, Jukema JW, Biessen EA. CC chemokine ligand-5 (CCL5/RANTES) and CC chemokine ligand-18 (CCL18/PARC) are specific markers of refractory unstable angina pectoris and are transiently raised during severe ischemic symptoms. Circulation. 2007; 116: 1931-41.

37. Tuomainen AM, Nyyssönen K, Laukkanen JA, Tervahartiala T, Tuomainen TP, Salonen JT, Sorsa T, Pussinen PJ. Serum matrix metalloproteinase- 8 concentrations are associated with cardiovascular outcome in men. Arterioscler Thromb Vasc Biol. 2007; 27: 2722-8.

38. Durán MC, Boeri-Erba E, Mohammed S, Martín-Ventura JL, Egido J, Vivanco F, Jensen ON. Characterization of HSP27 phosphorylation sites in human atherosclerotic plaque secretome. Methods Mol Biol. 2007; 357: 151-63.

39. Durán MC, Martín-Ventura JL, Mohammed S, Barderas MG, Blanco-Colio LM, Mas S, Moral V, Ortega L, Tuñón J, Jensen ON, Vivanco F, Egido J. Atorvastatin modulates the profile of proteins released by human atherosclerotic plaques. Eur J Pharmacol. 2007; 562: 119-29.

40. Cleutjens KB, Faber BC, Rousch M, van Doorn R, Hackeng TM, Vink C, Geusens P, ten Cate H, Waltenberger J, Tchaikovski V, Lobbes M, Somers V, Sijbers A, Black D, Kitslaar PJ, Daemen MJ. Noninvasive diagnosis of ruptured peripheral atherosclerotic lesions and myocardial infarction by antibody profiling. J Clin Invest. 2008; 118: 297985.

41. Blanco-Colio LM, Martín-Ventura JL, Vivanco F, Michel JB, Meilhac O, Egido J. Biology of atherosclerotic plaques: what we are learning from proteomic analysis. Cardiovasc Res. 2006; 72: 18-29. 
42. Wilson AM, Kimura E, Harada RK, Nair N, Narasimhan B, Meng XY, Zhang F, Beck KR, Olin JW, Fung ET, Cooke JP. Beta2-microglobulin as a biomarker in peripheral arterial disease: proteomic profiling and clinical studies. Circulation. 2007; 116: 1396-403.

43. Bayes-Genis A, Conover CA, Overgaard MT, Bailey KR, Christiansen M, Holmes DR Jr, Virmani R, Oxvig C, Schwartz RS. Pregnancy-associated plasma protein A as a marker of acute coronary syndromes. N Engl J Med. 2001; 345:1022-9.

44. Dominguez-Rodriguez A, Abreu-Gonzalez P, Garcia-Gonzalez M, Ferrer J, Vargas M. Circulating pregnancy-associated plasma protein $\mathrm{A}$ is not an early marker of acute myocardial infarction. Clin Biochem. 2005; 38: 180-2.

45. Qin QP, Wittfooth S, Pettersson K. Measurement and clinical significance of circulating PAPP-A in ACS patients. Clin Chim Acta. 2007; 380: 59-67.

46. Pepe MS, Thompson ML. Combining diagnostic test results to increase accuracy. Biostatistics. 2000; 1: 123-40.

47. Pencina MJ, D'Agostino RB. Overall $\mathrm{C}$ as a measurement of discrimination in survival analysis: model specific population value and confidence interval estimation. Stat Med. 2004; 23: 2109-23.

48. Varo N, de Lemos JA, Libby P, Morrow DA, Murphy SA, Nuzzo R, Gibson CM, Cannon CP, Braunwald E, Schönbeck U. Soluble CD40L: risk prediction after acute coronary syndromes. Circulation. 2003; 108: 1049-52.

49. Ardigo D, Assimes TL, Fortmann SP, Go AS, Hlatky M, Hytopoulos E, Iribarren C, Tsao PS, Tabibiazar R, Quertermous T; ADVANCE Investigators. Circulating chemokines accurately identify individuals with clinically significant atherosclerotic heart disease. Physiol Genomics. 2007; 31: 402-9.

50. Baldus S, Heitzer T, Eiserich JP, Lau D, Mollnau H, Ortak M, Petri S, Goldmann B, Duchstein HJ, Berger J, Helmchen U, Freeman BA, Meinertz T, Münzel T. Myeloperoxidase enhances nitric oxide catabolism during myocardial ischemia and reperfusion. Free Radic Biol Med. 2004; 37: 902-11.

51. Falk E, Fernández-Ortiz A. Role of thrombosis in atherosclerosis and its complications. Am J Cardiol. 1995; 75: 3B-11B.

52. Virmani R, Kolodgie FD, Burke AP, Farb A, Schwartz SM. Lessons from sudden coronary death: a comprehensive morphological classification scheme for atherosclerotic lesions. Arterioscler Thromb Vasc Biol. 2000; 20: 1262-75.

53. Moreno PR, Purushothaman KR, Zias E, Sanz J, Fuster V. Neovascularization in human atherosclerosis. Curr Mol Med. 2006; 6: 457-77.

54. Sanz J, Fayad ZA. Imaging of atherosclerotic cardiovascular disease. Nature. 2008; 451: 953-7.

55. Tan KT, Lip GY. Imaging of the unstable plaque. Int J Cardiol. 2008; 127: 157-65.

56. Kwee RM, van Oostenbrugge RJ, Hofstra L, Teule GJ, van Engelshoven JM, Mess WH, Kooi ME. Identifying vulnerable carotid plaques by noninvasive imaging. Neurology. 2008; 70: 2401-9.

57. de Bray JM, Baud JM, Delanoy P, Camuzat JP, Dehans V, Descamp-Le Chevoir J, Launay JR, Luizy F, Sentou Y, Cales P. Reproducibility in ultrasonic characterization of carotid plaques. Cerebrovasc Dis. 1998; 8: 273-7.

58. Sabetai MM, Tegos TJ, Nicolaides AN, Dhanjil S, Pare GJ, Stevens JM. Reproducibility of computer-quantified carotid plaque echogenicity: can we overcome the subjectivity? Stroke. 2000; 31: 2189-96.

59. Prabhakaran S, Rundek T, Ramas R, Elkind MS, Paik MC, Boden-Albala B, Sacco RL. Carotid plaque surface irregularity predicts ischemic stroke: the northern Manhattan study. Stroke. 2006; 37: 2696-701. 
60. Kitamura A, Iso H, Imano H, Ohira T, Okada T, Sato S, Kiyama M, Tanigawa T, Yamagishi K, Shimamoto T. Carotid intima-media thickness and plaque characteristics as a risk factor for stroke in Japanese elderly men. Stroke. 2004; 35: 2788-94.

61. Mori S, Endo M, Kohno R, Minohara S. Improves motion compensation in 3D-CT using respiratory-correlated segment reconstruction: diagnostic and radiotherapy implications. Br J Radiol. 2006; 79: 745-55.

62. Mori S, Endo M, Nishizawa K, Murase K, Fujiwara H, Tanada S. Comparison of patient doses in 256-slice CT and 16-slice CT scanners. Br J Radiol. 2006; 79: 56-61.

63. Funabashi N, Mizuno N, Yoshida K, Tsunoo T, Mori S, Tanada S, Endo M, Komuro I. Superiority of synchrony of 256-slice cone beam computed tomography for acquiring pulsating objects. Comparison with conventional multislice computed tomography. Int J Cardiol. 2007; 118: 400-5.

64. de Weert TT, Ouhlous M, Meijering E, Zondervan PE, Hendriks JM, van Sambeek MR, Dippel DW, van der Lugt A. In vivo characterization and quantification of atherosclerotic carotid plaque components with multidetector computed tomography and histopathological correlation. Arterioscler Thromb Vasc Biol. 2006; 26: 2366-72.

65. Fayad ZA, Fuster V. Clinical imaging of the high-risk or vulnerable atherosclerotic plaque. Circ Res. 2001; 89: 305-16.

66. Miserus RJJHM, Heeneman S, Van Engelshoven JMA, Kooi ME, Daemen MJAP. Development and validation of novel imaging technologies to assist translational studies in atherosclerosis. Drug Discovery Today: Technologies. 2006; 32: 195-204.

67. Brenner DJ, Hall EJ. Computed tomography: an increasing source of radiation exposure. N Engl J Med. 2007; 357: 2277-84.

68. Tawakol A, Migrino RQ, Hoffmann U, Abbara S, Houser S, Gewirtz H, Muller JE, Brady TJ, Fischman AJ. Noninvasive in vivo measurement of vascular inflammation with F-18 fluorodeoxyglucose positron emission tomography. J Nucl Cardiol. 2005; 12: 294-301.

69. Rudd JH, Myers KS, Bansilal S, Machac J, Pinto CA, Tong C, Rafique A, Hargeaves R, Farkouh M, Fuster V, Fayad ZA. Atherosclerosis inflammation imaging with 18F-FDG PET: carotid, iliac, and femoral uptake reproducibility, quantificantion methods, and recommendations. J Nucl Med. 2008; 49: 871-8.

70. Arauz A, Hoyos L, Zenteno M, Mendoza R, Alexanderson E. Carotid plaque inflammation detected by $18 \mathrm{~F}$-fluorodeoxyglucose-positron emission tomography. Pilot study. Clin Neurol Neurosurg. 2007; 109: 409-12.

71. Laufer EM, Reutelingsperger CP, Narula J, Hofstra L. Annexin A5: an imaging biomarker of cardiovascular risk. Basic Res Cardiol. 2008; 103: 95-104.

72. Kietselaer BL, Reutelingsperger CP, Heidendal GA, Daemen MJ, Mess WH, Hofstra L, Narula J. Noninvasive detection of plaque instability with use of radiolabeled annexin A5 in patients with carotid-artery atherosclerosis. N Engl J Med. 2004; 350: 1472-3.

73. Solomon GJ, Rosen PP, Wu E. The role of gadolinium in triggering nephrogenic systemic fibrosis/nephrogenic fibrosing dermopathy. Arch Pathol Lab Med. 2007; 131: 1515-6.

74. Leiner T, Herborn CU, Goyen M. Nephrogenic systemic fibrosis is not exclussively associated with gadodiamide. Eur Radiol. 2007; 17: 1921-3.

75. Toussaint JF, LaMuraglia GM, Southern JF, Fuster V, Kantor HL. Magnetic resonance images lipid, fibrous, calcified, hemorrhagic, and thrombotic components of human atherosclerosis in vivo. Circulation. 1996; 94: 932-8. 
76. Yuan C, Mitsumori LM, Ferguson MS, Polissar NL, Echelard D, Ortiz G, Small R, Davies JW, Kerwin WS, Hatsukami TS. In vivo accuracy of multispectral magnetic resonance imaging for identifying lipid-rich necrotic cores and intraplaque hemorrhage in advanced human carotid plaques. Circulation. 2001; 104: 2051-6.

77. Shinnar M, Fallon JT, Wehrli S, Levin M, Dalmacy D, Fayad ZA, Badimon JJ, Harrington M, Harrington E, Fuster V. The diagnostic accuracy of ex vivo MRI for human atherosclerotic plaque characterization. Arterioscler Thromb Vasc Biol. 1999; 19: 275661.

78. Finn JP, Edelman RR. Black-blood and segmented k-space magnetic resonance angiography. Magn Reson Imaging Clin N Am. 1993; 1: 348-57.

79. Wang J, Yarnykh VL, Hatsukami T, Chu B, Balu N, Yuan C. Improves suppression of plaque-mimicking artifacts in black-blood carotid atherosclerosis imaging using a multislice motion-sensitized, driven-equilibrium (MSDE) turbo spin echo (TSE) sequence. Magn Reson Med. 2007; 58: 973-81.

80. Mitsumori LM, Hatsukami TS, Ferguson MS, Kerwin WS, Cai J, Yuan C. In vivo accuracy of multisequence MR imaging for identifying unstable fibrous caps in advanced human carotid plaques. J Magn Reson Imaging. 2003; 17: 410-20.

81. Hatsukami TS, Ross R, Polissar NL, Yuan C. Visualization of fibrous cap thickness and rupture in human atherosclerotic carotid plaque in vivo with high-resolution magnetic resonance imaging. Circulation. 2000; 102: 959-64.

82. Moody AR, Murphy RE, Morgan PS, Martel AL, Delay GS, Allder S, MacSweeney ST, Tennant WG, Gladman J, Lowe J, Hunt BJ. Characterization of complicated carotid plaque with magnetic resonance direct thrombus imaging in patients with cerebral ischemia. Circulation. 2003; 107: 3047-52.

83. Cappendijk VC, Cleutjens KB, Heeneman S, Schurink GW, Welten RJ, Kessels AG, van Suylen RJ, Daemen MJ, van Engelshoven JM, Kooi ME. In vivo detection of hemorrhage in human atherosclerotic plaques with magnetic resonance imaging. J Magn Reson Imaging. 2004; 20: 105-10.

84. Cappendijk VC, Heeneman S, Kessels AG, Cleutjens KB, Schurink GW, Welten RJ, Mess WH, van Suylen RJ, Leiner T, Daemen MJ, van Engelshoven JM, Kooi ME. Comparison of single-sequence T1w TFE MRI with multisequence MRI for the quantification of lipid-rich necrotic core in atherosclerotic plaque. J Magn Reson -Imaging. 2008; 27: 1347-55.

85. Cappendijk VC, Kessels AG, Heeneman S, Cleutjens KB, Schurink GW, Welten RJ, Mess WH, van Suylen RJ, Leiner T, Daemen MJ, van Engelshoven JM, Kooi ME. Comparison of lipid-rich necrotic core size in symptomatic and asymptomatic carotid atherosclerotic plaque: Initial results. J Magn Reson Imaging. 2008; 27: 1356-61.

86. Yuan C, Miller ZE, Cai J, Hatsukami T. Carotid atherosclerotic wall imaging by MRI. Neuroimaging Clin N Am. 2002; 12: 391-401.

87. Wasserman BA, Smith WI, Trout HH 3rd, Cannon RO 3rd, Balaban RS, Arai AE. Carotid artery atherosclerosis: in vivo morphologic characterization with gadoliniumenhanced double-oblique MR imaging initial results. Radiology. 2002; 223: 566-73.

88. Kerwin W, Hooker A, Spilker M, Vicini P, Ferguson M, Hatsukami T, Yuan C. Quantitative magnetic resonance imaging analysis of neovasculature volume in carotid atherosclerotic plaque. Circulation. 2003; 107: 851-6.

89. Kerwin WS, O'Brien KD, Ferguson MS, Polissar N, Hatsukami TS, Yuan C. Inflammation in carotid atherosclerotic plaque: a dynamic contrast-enhanced MR imaging study. Radiology. 2006; 241: 459-68. 
90. Kerwin WS, Oikawa M, Yuan C, Jarvik GP, Hatsukami TS. MR imaging of adventitial vasa vasorum in carotid atherosclerosis. Magn Reson Med. 2008; 59: 507-14.

91. Bulte JWM. Nanoparticles in biomedical imaging: emerging technologies and applications. 2008. Berlin, Germany: Springers.

92. Wang YX, Hussain SM, Krestin GP. Superparamagnetic iron oxide contrast agents: physiochemical characteristics and applications in MR imaging. Eur Radiol. 2001; 11: 2319-31.

93. Kooi ME, Cappendijk VC, Cleutjens KB, Kessels AG, Kitslaar PJ, Borgers M, Frederik PM, Daemen MJ, van Engelshoven JM. Accumulation of ultrasmall superparamagnetic particles of iron oxide in human atherosclerotic plaques can be detected by in vivo magnetic resonance imaging. Circulation. 2003; 107: 2453-8.

94. Trivedi RA, U-King-Im JM, Graves MJ, Cross JJ, Horsley J, Goddard MJ, Skepper JN, Quartey G, Warburton E, Joubert I, Wang L, Kirkpatrick PJ, Brown J, Gillard JH. In vivo detection of macrophages in human carotid atheroma: temporal dependence of ultrasmall superparamagnetic particles of iron oxide-enhanced MRI. Stroke. 2004; 35: 1631-5.

95. Trivedi RA, Mallawarachi C, U-King-Im JM, Graves MJ, Horsley J, Goddard MJ, Brown A, Wang L, Kirkpatrick PJ, Brown J, Gillard JH. Identifying inflamed carotid plaques using in vivo USPIO-enhanced MR imaging to label plaque macrophages. Arterioscler Thromb Vasc Biol. 2006; 26: 1601-6.

96. Barkhausen J, Ebert W, Heyer C, Debatin JF, Weinmann HJ. Detection of atherosclerotic plaque with Gadofluorine-enhanced magnetic resonance imaging. Circulation. 2003; 108: 605-9.

97. Sirol M, Itskovich VV, Mani V, Aguinaldo JG, Fallon JT, Misselwitz B, Weinmann HJ, Fuster V, Toussaint JF, Fayad ZA. Lipid-rich atherosclerotic plaques detected by Gadofluorine-enhanced in vivo magnetic resonance imaging. Circulation. 2004; 109: 2890-6.

98. Yasuda S, Ikuta K, Uwatoku T, Oi K, Abe K, Hyodo F, Yoshimitsu K, Sugimura K, Utsumi H, Katayama Y, Shimokawa $\mathrm{H}$. In vivo magnetic resonance imaging of atherosclerotic lesions with a newly developed Evans-blue-DTPA-gadolinium contrast medium in apolipoprotein-E-deficient mice. J Vasc Res. 2008; 45: 123-8.

99. Saam T, Underhill HR, Chu B, Takaya N, Cai J, Polissar NL, Yuan C, Hatsukami TS. Prevalence of American Heart Association type VI carotid atherosclerotic lesions identified by magnetic resonance imaging for different levels of stenosis as measured by duplex ultrasound. J Am Coll Cardiol. 2008; 51: 1014-21.

100. Altaf N, McSweeney ST, Gladman J, Auer DP. Carotid intraplaque hemorrhage predicts current symptoms in patients with high-grade carotid stenosis. Stroke. 2007; 38: 1633-5.

101. Altaf N, Daniels L, Morgan PS, Auer D, McSweeney ST, Moody AR, Gladman JR. Detection of intraplaque hemorrhage by magnetic resonance imaging in symptomatic patients with mild to moderate carotid stenosis predicts recurrent neurologic events. J Vasc Surg. 2008; 47: 337-42.

102. Parmelee DJ, Walovitch RC, Ouellet HS, Lauffer RB. Preclinical evaluation of the pharmacokinetics, biodistribution, and elimination of MS-325, a blood pool agent for magnetic resonance imaging. Invest Radiol. 2007; 32: 741-7.

103. Lauffer RB, Parmelee DJ, Dunham SU, Ouellet HS, Dolan RP, Witte S, McMurry TJ, Walovitch RC. MS-325: albumin-targeted contrast agent for MR angiography. Radiology. 1998; 207: 529-38.

104. Goyen M, Shamsi K, Schoenberg SO. Vasovist-enhanced MR angiography. Eur Radiol. 2006; 16 Suppl 2: B9-B14. 
105. Nicholson JP, Wolmarans MR, Park GR. The role of albumin in critical illness. Br J Anaesth. 2000; 85: 599-610.

106. Smith EB, Staples EM. Distribution of plasma proteins across the human aortic wall-barrier functions of endothelium and internal elastic lamina. Atherosclerosis. 1980; 37: $579-90$.

107. Londoño I, Leclerc Y, Bendayan M. Ultrastructural localization of endogenous albumin in human aortic tissue by protein A-gold immunocytochemistry. Am J Pathol. 1992; 140: 179-91.

108. Zhang Y, Cliff WJ, Schoefl GI, Higgins G. Immunohistochemical study of intimal microvessels in coronary atherosclerosis. Am J Pathol. 1993; 143: 164-72.

109. Cornily JC, Hyafil F, Calcagno C, Briley-Saebo KC, Tunstead J, Aguinaldo JG, Mani V, Lorusso V, Cavagna FM, Fayad ZA. Evaluation of neovessels in atherosclerotic plaques of rabbits using an albumin-binding intravascular contrast agent and MRI. J Magn Reson Imaging. 2008; 27: 1406-11.

110. Esposito L, Sievers M, Sander D, Heider P, Wolf O, Greil O, Zimmer C, Poppert H. Detection of unstable carotid artery stenosis using MRI. J Neurol. 2007; 254: 1714-22.

111. Anumula S, Song HK, Wright AC, Wehrli FW. High-resolution black-blood MRI of the carotid vessel wall using phased-array coils at 1.5 and 3 Tesla. Acad Radiol. 2005; 12: 1521-6.

112. Koktzoglou I, Chung YC, Mani V, Carroll TJ, Morasch MD, Mizsei G, Simonetti OP, Fayad ZA, Li D. Multislice dark-blood carotid artery wall imaging: a $1.5 \mathrm{~T}$ and $3.0 \mathrm{~T}$ comparison. J Magn Reson Imaging. 2006; 23: 699-705.

113. Hinton DP, Cury RC, Chan RC, Wald LL, Sherwood JB, Furie KL, Pitts JT, Schmitt F. Bright and black blood imaging of the carotid bifurcation at 3.0T. Eur J Radiol. 2006; 57: 403-11.

114. Yarnykh VL, Terashima M, Hayes CE, Shimakawa A, Takaya N, Nguyen PK, Brittain JH, McConnell MV, Yuan C. Multicontrast black-blood MRI of carotid arteries: comparison between 1.5 and 3 tesla magnetic field strengths. J Magn Reson Imaging. 2006; 23: 691-8.

115. Hinton-Yates DP, Cury RC, Wald LL, Wiggins GC, Keil B, Seethmaraju R, Gangadharamurthy D, Ogilvy CS, Dai G, Houser SL, Stone JR, Furie KL. 3.0 T plaque imaging. Top Magn Reson Imaging. 2007; 18: 389-400.

116. Thakur ML, Lentle BC. Joint SNM/RSNA Molecular Imaging Summit Statement. J Nucl Med. 2005; 46: 11N-13N, 42N.

117. Massoud TF, Gambhir SS. Molecular imaging in living subjects: seeing fundamental biological processes in a new light. Genes Dev. 2003; 17: 545-80.

118. Lanza GM, Abendschein DR, Yu X, Winter PM, Karukstis KK, Scott MJ, Fuhrhop RW, Scherrer DE, Wickline SA. Molecular imaging and targeted drug delivery with a novel, ligand-directed paramagnetic nanoparticle technology. Acad Radiol. 2002; 9 Suppl 2: S330-1.

119. Winter PM, Cai K, Caruthers SD, Wickline SA, Lanza GM. Emerging nanomedicine opportunities with perfluorocarbon nanoparticles. Expert Rev Med Devices. 2007; 4: $137-45$.

120. Mulder WJ, Strijkers GJ, van Tilborg GA, Griffioen AW, Nicolay K. Lipid-based nanoparticles for contrast-enhanced MRI and molecular imaging. NMR Biomed. 2006; 19: $142-64$.

121. Mulder WJ, Douma K, Koning GA, van Zandvoort MA, Lutgens E, Daemen MJ, Nicolay K, Strijkers GJ. Liposome-enhanced MRI of neointimal lesions in the ApoEKO mouse. Magn Reson Med. 2006; 55: 1170-4. 
122. Mulder WJ, Strijkers GJ, Briley-Saboe KC, Frias JC, Aguinaldo JG, Vucic E, Amirbekian V, Tang C, Chin PT, Nicolay K, Fayad ZA. Molecular imaging of macrophages in atherosclerotic plaques using bimodal PEG-micelles. Magn Reson Med. 2007; 58: 1164-70.

123. Prinzen L, Miserus RJ, Dirksen A, Hackeng TM, Deckers N, Bitsch NJ, Megens RT, Douma K, Heemskerk JW, Kooi ME, Frederik PM, Slaaf DW, van Zandvoort MA, Reutelingsperger CP. Optical and magnetic resonance imaging of cell death and platelet activation using annexin a5-functionalized quantum dots. Nano Lett. 2007; 7: 93-100.

124. Winter PM, Morawski AM, Caruthers SD, Fuhrhop RW, Zhang H, Williams TA, Allen JS, Lacy EK, Robertson JD, Lanza GM, Wickline SA. Molecular imaging of angiogenesis in early-stage atherosclerosis with alpha(v)beta3-integrin-targeted nanoparticles. Circulation. 2003; 108: 2270-4.

125. Winter PM, Neubauer AM, Caruthers SD, Harris TD, Robertson JD, Williams TA, Schmieder AH, Hu G, Allen JS, Lacy EK, Zhang H, Wickline SA, Lanza GM. Endothelial alpha(v)beta3 integrin-targeted fumagillin nanoparticles inhibit angiogenesis in atherosclerosis. Arterioscler Thromb Vasc Biol. 2006; 26: 2103-9.

126. Mulder WJ, van der Schaft DW, Hautvast PA, Strijkers GJ, Koning GA, Storm G, Mayo KH, Griffioen AW, Nicolay K. Early in vivo assessment of angiostatic therapy efficacy by molecular MRI. FASEB J. 2007; 21: 387-83.

127. Flacke S, Fischer S, Scott MJ, Fuhrhop RJ, Allen JS, McLean M, Winter P, Sicard GA, Gaffney PJ, Wickline SA, Lanza GM. Novel MRI contrast agent for molecular imaging of fibrin: implications for detecting vulnerable plaques. Circulation. 2001; 104: 1280-5.

128. Botnar RM, Perez AS, Witte S, Wiethoff AJ, Laredo J, Hamilton J, Quist W, Parsons EC Jr, Vaidya A, Kolodziej A, Barrett JA, Graham PB, Weisskoff RM, Manning WJ, Johnstone MT. In vivo molecular imaging of acute and subacute thrombosis using a fibrin-binding magnetic resonance imaging contrast agent. Circulation. 2004; 109: 2023-9.

129. Spuentrup E, Katoh M, Wiethoff AJ, Buecker A, Botnar RM, Parsons EC, Guenther RW. Molecular coronary MR imaging of human thrombi using EP-2104R, a fibrintargeted contrast agent: experimental study in a swine model. Rofo. 2007; 179: 1166-73.

130. Spuentrup E, Katoh M, Buecker A, Fausten B, Wiethoff AJ, Wildberger JE, Haage P, Parsons EC Jr, Botnar RM, Graham PB, Vettelschoss M, Günther RW. Molecular MR imaging of human thrombi in a swine model of pulmonary embolism using a fibrinspecific contrast agent. Invest Radiol. 2007; 42: 586-95.

131. Spuentrup E, Botnar RM, Wiethoff AJ, Ibrahim T, Kelle S, Katoh M, Ozgun M, Nagel E, Vymazal J, Graham PB, Günther RW, Maintz D. MR imaging of thrombi using EP2104R, a fibrin-specific contrast agent: initial results in patients. Eur Radiol. 2008; 18: 1995-2005.

132. Lancelot E, Amirbekian V, Brigger I, Raynaud JS, Ballet S, David C, Rousseaux O, Le Greneur S, Port M, Lijnen HR, Bruneval P, Michel JB, Ouimet T, Roques B, Amirbekian S, Hyafil F, Vucic E, Aguinaldo JG, Corot C, Fayad ZA. Evaluation of matrix metalloproteinases in atherosclerosis using a novel noninvasive imaging approach. Arterioscler Thromb Vasc Biol. 2008; 28: 425-32.

133. Kelly KA, Nahrendorf M, Yu AM, Reynolds F, Weissleder R. In vivo phage display selection yields atherosclerotic plaque targeted peptides for imaging. Mol Imaging Biol. 2006; 8: 201-7.

134. Megens RT, Oude Egbrink MG, Cleutjens JP, Kuijpers MJ, Schiffers PH, Merkx M, Slaaf DW, van Zandvoort MA. Imaging collagen in intact viable healthy and atherosclerotic arteries using fluorescently labeled CNA35 and two-photon laser scanning microscopy. Mol Imaging. 2007; 6: 247-60. 
Chapter 6

135. Garćia-Palmieri MR. The endothelium in health and in cardiovascular disease. P R Health Sci J. 1997; 16: 136-41.

136. Hong HY, Lee HY, Kwak W, Yoo J, Na MH, So IS, Kwon TH, Park HS, Huh S, Oh GT, Kwon IC, Kim IS, Lee BH. Phage Display Selection of Peptides that Home to Atherosclerotic Plaques: IL-4 Receptor as a Candidate Target in Atherosclerosis. J Cell Mol Med. 2007; Epub ahead of print.

137. Tran TD, Caruthers SD, Hughes M, Marsh JN, Cyrus T, Winter PM, Neubauer AM, Wickline SA, Lanza GM. Clinical applications of perfluorocarbon nanoparticles for molecular imaging and targeted therapeutics. Int J Nanomedicine. 2007; 2: 515-26.

138. Wickline SA, Lanza GM. Nanotechnology for molecular imaging and targeted therapy. Circulation. 2003; 107: 1092-5.

139. Center for Translational and Molecular Medicine. www.ctmm.nl. 2008. Eindhoven, The Netherlands.

140. Rosamond W, Flegal K, Furie K, Go A, Greenlund K, Haase N, Hailpern SM, Ho M, Howard V, Kissela B, Kittner S, Lloyd-Jones D, McDermott M, Meigs J, Moy C, Nichol G, O'Donnell C, Roger V, Sorlie P, Steinberger J, Thom T, Wilson M, Hong Y; American Heart Association Statistics Committee and Stroke Statistics Subcommittee. Heart disease and stroke statistics--2008 update: a report from the American Heart Association Statistics Committee and Stroke Statistics Subcommittee. Circulation. 2008; 117: e25-e146. 


\section{Summary}

Atherosclerosis remains one of the leading causes of death in industrialized countries. It is characterized by the accumulation of lipid and inflammatory components in the vessel wall which results in the formation of atherosclerotic plaques. Clinical events, such as myocardial infarction, angina pectoris, and stroke, are mainly the result of either progressive stenosis or rupture of these plaques.

In order to identify patients at high risk for future cardiovascular events, several risk models, such as MONICA or Framingham, have been developed. These models are based on well-known cardiovascular risk factors. However, they do not include new and emerging risk factors of cardiovascular disease (e.g. biomarkers) and they do not assess the risk of plaque rupture. Therefore, these models are able to assess long-term outcome of a certain population, but fail to assess the individual's near-future risk of developing cardiovascular complications.

A panel of experts lead by Naghavi devised a speculative roadmap, in which patients with high risk of developing near-future cardiovascular events (the 'vulnerable' patients) can be identified. This roadmap emphasized the need for the development of non-invasive, inexpensive and accurate screening tools. In the present thesis, we focused mainly on two aspects of this roadmap, namely non-invasive risk assessment (i.e. validation of (potential) biomarkers) and contrast-enhanced magnetic resonance imaging of atherosclerotic plaques.

\section{$V$ alidation of established biomarkers for cardiovascular disease}

In chapter 2, a systematic review of available literature was performed to assess the predictive value of sCD40L, IL-6, and oxLDL compared to more 
established biomarkers such as CRP and fibrinogen. SCD40L, IL-6, and oxLDL have all proven to be major players in atherogenesis and recent publications demonstrated their potential as biomarkers for predicting future cardiovascular events. In this review, we demonstrated that sCD40L, IL-6, and oxLDL had comparable relative risk estimates for cardiovascular disease when compared with CRP and fibrinogen (relative risk estimates in the order of 1.4). One of the drawbacks were the heterogeneous study designs of the evaluated literature, which hampered data pooling of the different studies. Finally, we suggested that future studies should have more homogenous study designs in order to enable data pooling and we emphasized the need of a multi-marker approach (in which a certain 'panel' of biomarkers is investigated for its predictive value in cardiovascular disease) in order to combine the strength of multiple biomarkers.

\section{$V$ alidation of new and emerging biomarkers for cardiovascular disease}

In chapter 3, we evaluated the new cardiovascular biomarkers MPO and PAPP-A in a cohort of 120 patients divided in patients with acute myocardial infarction, stable angina pectoris, unstable angina pectoris, and healthy controls. The prognostic value of these new markers was compared with the well-validated biomarkers leukocyte counts, fibrinogen, and CRP. Additional analysis of these biomarkers was performed after 6 months of followup. Compared to controls, leukocyte counts and serum concentrations of fibrinogen, CRP, PAPP-A (tested with an ultrasensitive research kit), and MPO were significantly increased in patients with acute myocardial infarction. Leukocyte counts and serum concentrations of MPO were also significantly increased in patients with unstable angina pectoris compared with controls. Serum concentrations of PAPP-A did not differ between study groups when the regular PAPP-A ELISA kit was used. The commercially available kits used to determine PAPP-A concentrations do not assess the serum concentrations of PAPP-A uncomplexed with proMBP, which is the subform of PAPP-A that is specific for atherosclerotic disease. They only assess the total amount of PAPP-A. The research ELISA kit for the detection of PAPP-A uses a monoclonal antibody specific for the PAPP-A uncomplexed with proMBP, whereas the regular PAPP-A kit uses a polyclonal, non-specific antibody. An additional discriminant analysis showed that leukocyte counts, MPO, and PAPP-A discriminated best between study groups. After six months, leukocyte counts and MPO were still increased in patient with acute myocardial infarction compared with controls. 
Contrast-enhanced magnetic resonance imaging of rabbit aortic atherosclerosis with the albumin-binding contrast agent gadofosveset

In chapter 4, we demonstrated the potential of the albumin-binding contrast agent gadofosveset for the depiction of atherosclerotic vessels with MRI. In this study, gadofosveset $(0.05 \mathrm{mmol} / \mathrm{kg})$ was compared with (the clinically widely available) Gd-DTPA (0.5 mmol $/ \mathrm{kg})$ in both atherosclerotic and normal rabbit aortas. The dose was adjusted for the higher $\mathrm{r}_{1}$ relaxivity of gadofosveset. After the final MRI experiments, rabbits were sacrificed and the aorta was harvested. The acquired samples were subsequently HE stained, and immunohistochemically stained for the presence of microvessels and albumin. In addition, PIXE analysis was performed to analyze the concentration and distribution of gadolinium within the vessel walls. In this study, we demonstrated that gadofosveset resulted in a significantly higher signal enhancement in the MR images of the atherosclerotic vessel wall when compared with controls. There was no significant difference in vessel wall signal enhancement of atherosclerotic and normal rabbits receiving GdDTPA. Immunohistochemical staining for micro-vessels and albumin showed increased amount of micro-vessels and albumin content in atherosclerotic rabbits when compared with control animals. PIXE analysis showed that equal amounts of gadolinium were present in atherosclerotic and control rabbits receiving gadofosveset. We hypothesized that binding of gadofosveset to intraplaque albumin resulted in more signal enhancement of atherosclerotic vessels. An equal amount of gadolinium was found in atherosclerotic and control rabbits receiving Gd-DTPA. In addition, when corrected for the higher dose of Gd-DTPA, the gadolinium content of the vessel walls were comparable to the gadofosveset groups. Furthermore, we found a significant positive correlation between MR signal enhancement and both increased microvessel density and intraplaque albumin content. These findings suggested that gadofosveset-enhanced MR imaging of atherosclerosis might have potential for imaging of human atherosclerotic vessel walls.

\section{Contrast-enhanced magnetic resonance imaging of human carotid atherosclerosis with the albumin-binding contrast agent gadofosveset}

In chapter 5, we demonstrated that patients scheduled for carotid endarterectomy (CEA) showed variable signal enhancement of the carotid artery plaque 24 hours after administration of gadofosveset. A subanalysis performed on these data revealed a significantly larger MR signal enhancement in symptomatic patients when compared with asymptomatic patients. Histo- 
logical analysis of carotid artery plaques removed during subsequent CEA showed no correlation between MR signal enhancement and various plaque components. In addition, no correlation between MR signal enhancement and intraplaque microvessel density was observed. Since the adventitia is not removed during CEA the adventitial microvessel density could not be assessed. Nonetheless, there was a significant correlation between MR signal enhancement and intraplaque albumin content, suggesting that the increased amount of intraplaque albumin, probably resulting from influx via (leaky) adventitial or intraplaque microvessels can possibly explain these findings.

\section{General discussion}

In chapter 6 , the results of this thesis are discussed with respect to the current available literature on biomarkers of cardiovascular disease and noninvasive imaging of atherosclerotic lesions. Future applications and developments in these fields of research are additionally discussed.

\section{Conclusion and outlook}

In this thesis, we demonstrated that established biomarkers for cardiovascular disease (such as CRP, fibrinogen, sCD40L, IL-6, and oxLDL) have low to moderate relative risk estimates for predicting future cardiovascular events. Of the biomarkers tested in this thesis, we demonstrated that MPO, PAPP-A, and leukocyte counts have the most potential in predicting future cardiovascular complications. In future studies, there is a clear need for comparable study designs in order to pool available data on biomarkers in large meta-analyses, and we recommend a multi-marker approach for predicting cardiovascular disease.

In addition, this thesis showed that the albumin-binding contrast agent gadofosveset has high potential in MRI of atherosclerosis, since initial results showed that it was able to distinguish between atherosclerotic and normal rabbit vessel wall, and symptomatic and asymptomatic patients with significant carotid artery stenosis. We believe that plaque imaging applications of gadofosveset hold great potential. Future studies should investigate the possibility to identify patients at risk for future cardiovascular events (such as stroke) by using gadofosveset-enhanced MR imaging, especially in patients with $30-69 \%$ carotid stenosis who are not scheduled for carotid surgery.

The clinical values of biomarkers of cardiovascular disease still need to be investigated in large clinical trials. Both research on these biomarkers and 
non-invasive magnetic resonance imaging of atherosclerosis are still in their formative years. New technological improvements are continuously introduced, leading to more biomarkers becoming available and more sophisticated methods of imaging the atherosclerotic disease process. Hopefully, in the future this will lead to improved assessment of the individual's risk of developing cardiovascular complications and will aid in the identification of individuals at risk in the general population. 



\section{Nederlandse samenvatting (Dutch summary)}

Atherosclerose ('aderverkalking') is één van de belangrijkste doodsoorzaken in geïndustrialiseerde landen. De aandoening wordt gekenmerkt door een opeenhoping van vetten en ontstekingscellen in de wanden van slagaders, wat na verloop van tijd resulteert in de ontwikkeling van een zogenaamde atherosclerotische plaque. Klinische complicaties van atherosclerose, zoals bijvoorbeeld een hartinfarct, angina pectoris (pijn op de borst in rust of bij inspanning) of een beroerte, zijn voornamelijk het gevolg van een toenemende vernauwing van de bloedvaten en/of het scheuren van de plaques.

Verschillende risicomodellen, zoals die bijvoorbeeld in de MONICA of Framingham studie beschreven zijn, werden in het verleden ontwikkeld om individuen met een groot risico op het krijgen van hart- en vaatziekten te kunnen identificeren. Deze modellen zijn voornamelijk gebaseerd op reeds bekende risicofactoren. $\mathrm{Zij}$ maken echter geen gebruik van recent ontdekte risicofactoren voor hart- en vaatziekten (bijvoorbeeld op basis van bloedmonsters door meting van bepaalde moleculen, zogenaamde biomarkers, waarmee men progressie van een bepaalde ziekte of het effect van behandeling ervan kan evalueren) en zij geven geen inschatting van het risico van het scheuren van een individuele plaque. Deze modellen kunnen daarom de uitkomst van een bepaalde groep mensen op de lange termijn inschatten, maar zij zeggen niets over het korte-termijn risico op hart- en vaatziekten van een bepaald individu.

Een aantal experts onder leiding van Naghavi stelde daarom een speculatieve beslisboom op, waarmee patiënten met een hoog risico op het ontwikkelen van hart- en vaatziekten in de nabije toekomst kunnen worden geïdentificeerd. Zij benadrukten de noodzaak voor de ontwikkeling van niet- 
invasieve, goedkope en nauwkeurige screeningsmethoden. In dit proefschrift wordt ingegaan op twee aspecten van deze beslisboom, namelijk de validiteit van potentiële biomarkers ten behoeve van niet-invasieve risicoinschatting en contrast-versterkte magnetische resonantie beeldvorming van atherosclerotische plaques.

\section{$V$ alidatie van bekende biomarkers voor hart-en vaatziekten}

In hoofdstuk 2 werd een systematisch overzicht gegeven van de voorspellende waarde van verschillende biomarkers (sCD40L, IL-6 en oxLDL) met betrekking tot hart- en vaatziekten. Deze biomarkers werden vergeleken met reeds uitgebreid onderzochte biomarkers zoals CRP en fibrinogeen. Van sCD40L, IL-6 en oxLDL is bekend dat zij een belangrijke rol spelen in de ontwikkeling van atherosclerotische plaques en de bijbehorende complicaties. Recente publicaties met betrekking tot deze biomarkers toonden hun potentieel als biomarker voor de voorspelling van het optreden van hart- en vaatziekten reeds aan. In dit hoofdstuk toonden wij bovendien aan dat sCD40L, IL-6 en oxLDL een vergelijkbare relatieve risico-inschatting hadden voor hart- en vaatziekten als CRP en fibrinogeen (relatieve risicoinschatting in de grootte van 1.4). Omdat één van de tekortkomingen van de beschreven publicaties de diversiteit van de studieopzet was, wat de bundeling van data van de verschillende studies verhinderde, stelden wij voor dat toekomstige studies een meer homogene opzet dienden te hebben om bundeling van data wel mogelijk te maken. We benadrukten ook de noodzaak van een 'multimarker' benadering (waarin de voorspellende waarde voor hart- en vaatziekten van een bepaalde combinatie van biomarkers wordt onderzocht) om zodoende de kracht van meerdere biomarkers te kunnen bundelen.

\section{$V$ alidatie van nieune biomarkers voor hart- en vaatziekten}

In hoofdstuk 3 onderzochten wij de nieuwe cardiovasculaire biomarkers MPO en PAPP-A in een groep van 120 personen, onderverdeeld in patiënten met een acuut hartinfarct, stabiele angina pectoris, instabiele angina pectoris en gezonde vrijwilligers. Het potentieel als biomarker voor hart- en vaatziekten van deze nieuwe biomarkers werd in deze studie vergeleken met reeds gevestigde biomarkers zoals leukocyten, CRP en fibrinogeen. De concentraties van deze biomarkers werden ook gemeten na 6 maanden followup. In vergelijking tot de gezonde controlepersonen, hadden patiënten met een acuut hartinfarct significant hogere concentraties leukocyten, fibrinogeen, CRP, PAPP-A en MPO in hun bloed. De concentraties leukocyten en 
MPO waren tevens significant verhoogd bij patiënten met instabiele angina pectoris (vergeleken met de gezonde vrijwilligers). Wanneer de reguliere PAPP-A ELISA meetmethode werd gebruikt voor de analyses, kon geen verschil in PAPP-A-concentraties worden aangetoond tussen de verschillende groepen. De commercieel verkrijgbare meetmethoden voor het bepalen van PAPP-A-concentraties bepalen de totale concentratie PAPP-A, niet alleen de concentratie van PAPP-A die geen complex vormt met een eiwit in het bloed, het proMBP. Deze laatste vorm is juist de subvorm van PAPPA die specifiek is voor atherosclerose. De meeste gangbare commerciële meetmethoden bepalen enkel de totale concentratie van PAPP-A in het bloed. Een recent ontwikkelde nieuwe aanpassing in de methode om PAPPA te bepalen, meet specifiek het PAPP-A dat geen complex vormt met proMBP. Dit in tegenstelling tot de reguliere PAPP-A meetmethoden, die de totale concentratie PAPP-A bepalen. Een aanvullende statistische analyse toonde verder aan dat het onderscheid tussen de verschillende studiegroepen het best gemaakt werd met behulp van concentraties van leukocyten, MPO en PAPP-A. Na zes maanden waren de concentraties van leukocyten en MPO nog steeds verhoogd bij patiënten met een acuut hartinfarct (vergeleken met gezonde controlepersonen).

\section{Contrast-versterkte mri van atherosclerose in konijnenaorta's met behulp van gadofosveset}

In hoofdstuk 4 onderzochten wij het potentieel van het albumine-bindende contrastmiddel gadofosveset voor de analyse van atherosclerotische bloedvaten met behulp van magnetische resonantie beeldvorming (MRI). In deze studie maakten wij gebruik van konijnen die atherosclerotische plaques ontwikkelden in hun lichaamsslagader (aorta) en konijnen die geen atherosclerose ontwikkelden. $\mathrm{Na}$ binding van gadofosveset aan albumine, het meest voorkomende eiwit in de bloedbaan, neemt de relaxiviteit van dit contrastmiddel toe, resulterend in een hogere signaalintensiteit op magnetische resonantie afbeeldingen. In deze studie werd gadofosveset (0.05 $\mathrm{mmol} / \mathrm{kg}$ ) vergeleken met (het in de kliniek veel gebruikte contrastmiddel) Gd-DTPA $(0.5 \mathrm{mmol} / \mathrm{kg})$ in zowel atherosclerotische als gezonde konijnenaorta's. De dosis van gadofosveset was aanzienlijk lager om te corrigeren voor de hogere relaxiviteit van gadofosveset. $\mathrm{Na}$ de laatste MRIexperimenten werden de konijnen opgeofferd en werden de aorta's verzameld. De verkregen monsters werden vervolgens hematoxyline/eosine gekleurd om de verschillende plaquecomponenten te kunnen onderscheiden. Aanvullend werd zij de aanwezigheid van microvaten en albumine vastgesteld. Bovendien werd er een 'proton induced x-ray emission' (PIXE) analy- 
se uitgevoerd om de concentratie en distributie van gadolinium (wat zowel in gadofosveset als in Gd-DTPA voorkomt) in de bloedvatwanden te kunnen vaststellen. $\mathrm{Na}$ toediening van gadofosveset werd in deze studie een significant hogere signaalversterking gezien op de MR-afbeeldingen van atherosclerotische vaatwanden vergeleken met de gezonde vaatwanden. Er was geen verschil tussen de signaalversterking van atherosclerotische en gezonde vaatwanden na toediening van Gd-DTPA. Het bleek dat het aantal microvaatjes en de hoeveelheid albumine in de plaque groter was in de vaatwanden van atherosclerotische konijnen vergeleken met gezonde dieren. PIXE-analyse toonde aan dat vergelijkbare hoeveelheden gadolinium aanwezig waren in atherosclerotische en gezonde dieren die gadofosveset kregen. Daarom concludeerden wij dat binding van gadofosveset aan albumine in de plaque waarschijnlijk de oorzaak is van de hogere signaalversterking van de atherosclerotische vaatwanden. Ook de hoeveelheid in de vaatwand aanwezig gadolinium in dieren die Gd-DTPA kregen, verschilde niet tussen de atherosclerotische en gezonde dieren. De hoeveelheden gadolinium in deze groepen waren bovendien vergelijkbaar met de hoeveelheden die waargenomen werden in de groepen die gadofosveset kregen (na correctie van de hogere dosering van Gd-DTPA). In deze studie vonden wij bovendien een significant positieve correlatie tussen de MR-signaalversterking in de vaatwanden en zowel de dichtheid van de microvaatjes als de hoeveelheid albumine in de plaque. Deze bevindingen suggereren dat gadofosvesetversterkte magnetische resonantie beeldvorming van atherosclerose veelbelovend is bij het afbeelden van humane atherosclerotische vaatwanden.

\section{Contrast-versterkte mri van humane atherosclerose van de arteria carotis communis met behulp van het albumine-bindende contrastmiddel gadofosveset}

In hoofdstuk 5 lieten wij zien dat 24 uur na toediening van gadofosveset patiënten die gepland stonden voor een endarterectomie (chirurgische verwijdering van de plaque in de halsslagader) van de arteria carotis communis (de halsslagader) een variabele MR-signaalversterking vertoonden van de plaque. Een subanalyse toonde aan dat symptomatische patiënten (patiënten die recent kortdurende neurologische uitval of een beroerte hebben ondervonden) een significant hogere MR-signaalversterking van de plaque lieten zien dan asymptomatische patiënten (patiënten die geen neurologische uitval hebben ondervonden in hun voorgeschiedenis). Histologische analyse van de plaques van de halsslagader (verwijderd tijdens de operatie) lieten geen correlatie zien tussen MR-signaalversterking en de verschillende weefselcomponenten in de plaque. Bovendien werd er geen correlatie aangetoond tussen MR-signaalversterking en dichtheid van de microvaatjes in de plaque. 
Desalniettemin werd er een significante correlatie aangetoond tussen MRsignaalversterking en de hoeveelheid albumine in de plaque. Dit suggereerde dat de toegenomen hoeveelheid albumine in de plaque (waarschijnlijk als gevolg van een verhoogde instroming via (lekkende) adventitiële microvaatjes) deze bevindingen mogelijk zou kunnen verklaren. De adventitia is het buitenste deel van de halsslagader dat niet verwijderd wordt tijdens de operatie. De correlatie tussen MR-signaalversterking en de dichtheid van de adventitiële microvaatjes kon daarom niet worden onderzocht.

\section{Algemene discussie}

In hoofdstuk 6 werden de resultaten van dit proefschrift besproken in relatie tot de huidig beschikbare literatuur over biomarkers van cardiovasculaire aandoeningen en niet-invasieve beeldvorming van atherosclerotische plaques. Toekomstige toepassingen en ontwikkelingen in deze onderzoeksgebieden werden besproken in dit hoofdstuk.

\section{Conclusie en beschouwing}

In dit proefschrift toonden wij aan dat de gevalideerde biomarkers voor hart- en vaatziekten (zoals CRP, fibrinogeen, sCD40L, IL-6 en oxLDL) een lage tot gemiddelde relatieve risico-inschatting geven bij de voorspelling van toekomstige cardiovasculaire complicaties. Van alle biomarkers die getest

werden in het kader van dit proefschrift, hadden MPO, PAPP-A en leukocyten de grootste potentie om toekomstige cardiovasculaire complicaties te voorspellen. Er is een duidelijke noodzaak om in toekomstige studies een homogene studieopzet te gebruiken, zodat de beschikbare data uit deze studies gebundeld kunnen worden in grote meta-analyses. Bovendien bevelen wij een multimarker-benadering voor de voorspelling van hart- en vaatziekten aan.

Dit proefschrift toonde ook aan dat het albumine-bindende contrastmiddel gadofosveset een groot potentieel heeft bij de magnetische resonantie beeldvorming van atherosclerose. Initiële resultaten laten zien dat gadofosveset in staat was om onderscheid te maken tussen atherosclerotische en gezonde bloedvatwanden van konijnenaorta's, en tussen symptomatische en asymptomatische patiënten met significante vernauwing van de halsslagader. Wij zijn van mening dat toepassing van gadofosveset in beeldvorming van plaques veelbelovend is. Toekomstige studies zouden de mogelijkheid om patiënten met een hoog risico op cardiovasculaire complicaties (zoals be- 
roerte) te identificeren aan de hand van gadofosveset-versterkte magnetische resonantie beeldvorming moeten onderzoeken, met name in de groep patiënten met 30-69\% vernauwing die volgens de huidige richtlijnen niet profiteren van een chirurgische ingreep.

De klinische waarde van biomarkers voor hart- en vaatziekten moet nog onderzocht worden in grote klinische trials. Zowel het onderzoek naar deze biomarkers als het onderzoek naar non-invasieve magnetische resonantie beeldvorming van atherosclerose staat nog in de kinderschoenen. Nieuwe technologische verbeteringen worden continu geïntroduceerd, wat leidt tot de beschikbaarheid van meer biomarkers en beter ontwikkelde methoden om het atherosclerotisch proces af te beelden. Hopelijk zullen deze ontwikkelingen in de toekomst leiden tot een verbeterde inschatting van het individuele risico voor de ontwikkeling van cardiovasculaire complicaties en zal het helpen bij de identificatie van individuen in de algemene populatie die een groot risico lopen op het krijgen van hart- en vaatziekten en die dan vervolgens preventief behandeld kunnen worden. 


\section{Dankwoord (Acknowledgements)}

In de afgelopen jaren heb ik het voorrecht gehad om voor mijn studies tal van mensen te leren kennen. Ik wil hen hier bedanken.

Allereerst wil ik mijn familie bedanken. Lieve Marie Claire, bedankt voor je geduld en je luisterend oor. Ook jij hebt hier veel moeite ingestopt wanneer onze plannen weer eens niet doorgingen omdat ik moest scannen, of omdat ik aan mijn 'boekje' moest werken. Ik kan je niet genoeg danken voor je begrip hiervoor. Ben, kleine beer, je hebt weinig meegekregen van pappa's werk aan dit boekwerk, maar toch loop jii als rode draad door dit onderzoek heen. Jij en Claire zijn de redenen waarom ik 's ochtends vroeg op kan staan en ik de motivatie blijf houden om overal mijn best voor te doen. Pap en mam, ik heb nooit de kans gehad om jullie 'officieel' ergens voor te bedanken. Zonder jullie steun was het nooit mogelijk geweest om te bereiken wat ik bereikt heb. Ik draag dit boek speciaal aan jullie op, omdat jullie voor Mariëlle en mij altijd klaar staan. Mariëlle, Paul, Kim en Femke: soms had ik weinig tijd voor jullie, omdat opleiding en promotie nu eenmaal voor gingen. Ik hoop vanaf nu wat vaker langs te kunnen komen in Noordwijk, want familie is voor altijd.

Geachte professor Van Engelshoven, beste Jos. Bedankt dat je mij benaderde om onderzoek te komen doen nadat ik enkele malen had geïnformeerd voor een opleidingsplaats bij Radiologie. Ik zie je als het 'typical example' van een pater familias en ik denk dat velen binnen onze afdeling dat ook zo voelen. We zullen je missen op de werkvloer, maar ik wil je bij deze toch veel succes gunnen met je welverdiende pensioen. 
Geachte professor Daemen, beste Mat. Ook jou wil ik bedanken voor de kans die ik gekregen heb. Ik heb de afgelopen jaren veel mogen leren van jouw benadering van research en beschouw het als een eer en genoegen om onder jouw begeleiding gewerkt te mogen hebben.

Beste Eline, jouw onverzettelijke gevoel voor precisie heeft een belangrijke bijdrage geleverd aan dit proefschrift. Mijn dank daarvoor. Zonder jouw begeleiding was dit werk zeker van mindere kwaliteit geworden. Bedankt voor je inzet, ook voor al de uren die je buiten kantoortijd aan mij besteed hebt. Ik wens je veel sterkte en succes bij al je toekomstige wetenschappelijke werk.

Beste Sylvia, ik kreeg na enkele wisselingen van de wacht uiteindelijk jou als mijn vaste co-promotor en daar heb ik geen spijt van gehad. Ik had het gevoel dat ik met alle vragen bij je terecht kon en dat is in mijn ogen essentieel als je promovendi begeleidt. Je kwam altijd met goede ideeën op de proppen, maar gaf ook ruimte om mijn eigen ideeën uit te werken. Waar nodig stuurde jij bij.

Ik wil ook mijn dank uitspreken aan de beoordelingscommissie. Professor Hoeks, professor Hofstra, professor Bielen, professor Mess, professor Lodder, ik wil u hartelijk danken voor de tijd en moeite die u genomen heeft om mijn proefschrift te beoordelen.

Robbert-Jan, 'bunny boy' en 'partner in crime'. Ik kan een boekje apart schrijven over onze nachtelijke avonturen bij het scannen en onze tijd in het opberghok. In de nacht worden buitengesloten bij het CPV, ruiten inslaan, 'Worms' op de laptop, tassen met bier en kebab: we hebben het allemaal overleefd. Heel veel succes met je eigen promotie en je verhuizing!

Valeria, mijn statistische rots in de branding, ook jou wil ik erg bedanken voor je inzet en hulp bij mijn ingewikkelde vragen. Aangetrokken door jouw manier van uitleg zocht ik je hulp en jij maakte dat statistiek wat begrijpelijker voor me werd. Jouw hulp was zeer motiverend en hoog significant $(\mathrm{p}<0.001)$.

Patty, mijn tweede statistische rots. Bedankt voor je hulp in de aanpak van mijn review. Ik heb er erg veel van geleerd en vooral de klinische benadering van het verhaal heeft mij erg veel schrijfplezier bezorgd. Succes met al je toekomstige werk. 
Tim, jij had enkele jaren geleden een idee om met een nieuw contrastmiddeltje wat 'te doen'. Al snel hadden we een leger konijnen op de been en kon het werk beginnen. Jouw inzet voor de research is bewonderenswaardig, je humor op de werkvloer onmisbaar. Ik hoop dat ik nog lang veel van je kan leren en nog vaak met je samen mag werken.

Alle onderzoekers waarmee ik samengewerkt heb de afgelopen jaren wil ik natuurlijk ook bedanken. Jaap, Vera, Marlies, Frank, Suzanne, Karolien, Sanne, Max, Robert, Luc: bedankt! Ook zij die reeds vertrokken zijn wil ik succes wensen met hun carrière: Robert en Nils, succes. In dit rijtje wil ik ook de overige keldergasten bedanken voor hun hulp en adviezen: Walter, Marc, Jos en Gerrit: bedankt!

Niet vergeten wil ik de assistenten en stafleden van de Radiologie, mijn nieuwe collegae: Carsten, Christianne, Aliaa, Saskia, Marjolein, Shekar, Elleke, Vincent, Quido, Marianne, Rogier, Ulrich, Paulien, Suzanne, Carolien, Rick, Rob, Frans, Linda, Michiel, Ed, Riel, Regina, Carla, Dick, Wim, René, Khiam, Jan en Jan, Simon, Paul, Bert-Jan. Bedankt voor jullie geduld en interesse tijdens de laatste loodjes. Met name alle stafleden bedankt die mij meer dan tegemoet zijn gekomen toen ik enige overlap had van opleiding en promotieonderzoek. Professor Wildberger, nogmaals welkom binnen onze afdeling en dank dat u mij in de mogelijkheid stelde om mijn proefschrift af te ronden.

Heel veel mensen heb ik leren kennen in de afgelopen jaren. Ik kan hen niet allemaal apart bedanken, maar ik wil hen duidelijk maken dat dit werk niet alleen door mij is gedaan, maar dat hun inzet en motivatie er mede toe hebben geleid. In dit kader wil ik bedanken: Esther Lutgens, Kitty Cleutjens, Jolanda van Golde, professor Mark Post, professor Hugo ten Cate, Patricia Wijers, René van Oerle, Carine van Zandvoort, Lex Ruiters, Rob Welten, Simone Rouwette, cardiologen en verpleegkundingen van het Maaslandziekenhuis te Sittard, Marion Feijge, Johan Heemskerk, Hans Duimel, José Govers, Jos Stegen, Anique Jansen-Vrehen, Mat Rousch, Peter Mutsaers, Ruben Roijers, Nicolas Debernardi, Willy Rompelberg, alle medewerkers van de Centrale Proefdier Voorzieningen (maar met name May, Petra, Joyce, Allard, Harry en Richard). Als er nu nog mensen zijn die ik vergeten ben, bedenk dan dat dit geen kwade opzet is en wil ik bij deze ook jullie erg bedanken voor jullie hulp en inzet. 
Dankwoord

Tot slot: ze worden vaak vergeten, maar zij vormen de ruggengraat van al dit onderzoek. Bedankt, alle deelnemers van de humane studies voor hun interesse, geduld en enthousiasme! 


\section{Curriculum vitae}

Marcus Bernardus Irene Lobbes

Born November 21, 1977, Heerlen

Education and professional appointments

1990-1996 Sint-Janscollege, Hoensbroek, the Netherlands Gymnasium

1996-2002 Faculty of Medicine, Maastricht, the Netherlands Medicine

1999 Course 'Management in health care'

Leiden, the Netherlands

2002-2003 Maaslandziekenhuis, Sittard, the Netherlands

Resident Neurology

2003-2007 $\quad$ MUMC $^{+}$, Radiology, Maastricht, the Netherlands

$\mathrm{PhD}$ student (present thesis)

2007-present $\mathrm{MUMC}^{+}$, Radiology, Maastricht, the Netherlands

Resident Radiology

2002-present Medical Team Zolder, Zolder, Belgium

Extrication team, licensed by the FIA 



\section{List of publications}

Lobbes M, Lutgens E, Heeneman S, Kooi E, Van Engelshoven J, Daemen $\mathrm{M}$, Nelemans $\mathrm{P}$. Is there more than $\mathrm{C}$-reactive protein and fibrinogen? The prognostic value of soluble CD40 ligand, interleukin- 6 and oxidized lowdensity lipoprotein with respect to coronary and cerebral vascular disease. Atherosclerosis 2006; 187(1): 18-25 (impactfactor 4.287).

Cleutjens K, Faber B, Rousch M, Van Doorn R, Hackeng T, Vink C, Geusens P, Ten Cate H, Waltenberger J, Tchaikovski V, Lobbes M, Somers V, Sijbers A, Black D, Kitslaar P, Daemen M. Non-invasive diagnosis of ruptures peripheral atherosclerotic lesions and myocardial infarction by antibody profiling. Journal of Clinical Investigation 2008; 118(8): 2979-2985 (impactfactor 15.754).

Lobbes M, Miserus R, Heeneman S, Lima Passos V, Mutsaers P, Debernardi N, Misselwitz B, Post M, Daemen M, Van Engelshoven J, Leiner T, Kooi E. Contrast-enhanced magnetic resonance vessel wall imaging in a rabbit model of atherosclerosis: comparison of gadofosveset and GdDTPA. Radiology 2009; 250: 682-691 (impa ct factor 5.251).

Lobbes M, Kooi E, Lutgens E, Ruiters A, Lima Passos V, Braat S, Rousch M, Van Engelshoven J, Daemen M, Heeneman S. Leukocyte counts, myeloperoxidase, and pregnancy-associated plasma protein A as biomarkers for cardiovascular disease: old and new markers evalued. Submitted for publication.

Lobbes M, Heeneman S, Lima Passos V, Welten R, Kwee R, Van der Geest R, Wiethoff A, Caravan P, Misselwitz B, Daemen M, Van Engelshoven J, Leiner T, Kooi E. Gadofosveset-enhanced magnetic resonance imag- 
ing of human carotid atherosclerotic plaques: initial experience. Submitted for publication.

Miserus R, Herías V, Prinzen L, Lobbes M, Van Suylen R, Dirksen A, Hackeng T, Heemskerk J, Van Engelshoven J, Daemen M, Van Zandvoort M, Heeneman S, Kooi E. Molecular MRI of early thrombus formation using a bimodal $\alpha_{2}$-antiplasmin-based contrast agent. JACC Imaging, in press. 